\title{
Modelos Beta-Binomial/Poisson-Gama para contagens bivariadas repetidas
}

\author{
Mayra Ivanoff Lora
}

TESE APRESENTADA

$\mathrm{AO}$

Instituto de Matemática e Estatística

DA

Universidade de São Paulo

PARA

OBTENÇÃO DO TÍTULO

DE

Doutor EM CIÊNCIAS

Programa: Estatística

Orientador: Prof. Dr. Julio da Motta Singer

São Paulo, dezembro de 2008 


\title{
Modelos Beta-Binomial/Poisson-Gama para contagens bivariadas repetidas
}

\author{
Este exemplar corresponde à redação \\ final da tese devidamente corrigida \\ e defendida por Mayra Ivanoff Lora \\ e aprovada pela comissão julgadora.
}

São Paulo, 01 de dezembro de 2008.

Banca examinadora:

- Prof. Dr. Julio da Motta Singer (orientador) - IME-USP

- Prof. Dr. Gilberto Alvarenga Paula - IME-USP

- Profa. Dra. Hildete Prisco Pinheiro - UNICAMP

- Profa. Dra. Clarice Garcia Borges Demetrio - ESALQ

- Prof. Dr. Dalton Francisco de Andrade - UFSC 


\section{Agradecimentos}

Ao Professor Julio da Motta Singer, com quem tive o privilégio de trabalhar mais uma vez, pelo apoio, orientação e amizade, desde da época em que eu apenas assistia suas aulas na graduação como ouvinte.

Ao Dr. Andre de Felice e ao Professor Carlos Alberto de Bragança Pereira, sem os quais eu não teria chegado até a estatística.

Ao Professor Clóvis de Araújo Peres, por todo incentivo e amizade.

À Eliana Nakano pelos dados sobre os caramujos de última hora (e ao Professor Carlos Alberto, novamente, por ter me colocado em contato com ela).

Aos amigos do IME, pelas muitas horas de estudo e companheirismo, não podendo deixar de citar o Cléber (da Costa Figueiredo), amigo desde os tempos em que éramos ouvintes.

Ao Rodrigo (de Deus Reinaldo), pelos momentos alegres entre tantas listas e por esperar os quase 5 anos desse doutorado.

À minha mãe (Nadiejda Ivanoff) e minha vó (Zumar Gasi), pelo incentivo, apoio e carinho inesgotáveis. 


\section{Resumo}

Em Lora e Singer (Statistics in Medicine, 2008), propusemos um modelo BetaBinomial/Poisson p-variado para análise dos dados provenientes de um estudo que consistiu em contar o número de tentativas e acertos de um exercício manual com duração de um minuto realizado por doentes de Parkinson, antes e depois de um treinamento. O objetivo era verificar se o treinamento aumentava o número de tentativas e a porcentagem de acerto, o que destaca o aspecto bivariado do problema. Esse modelo leva tais características em consideração, usa uma distribuição adequada para dados de contagem e ainda acomoda a sobredispersão presente na contagem dos acertos. Como generalização, inicialmente, propomos um modelo Beta-Binomial/Poisson-Gama que acomoda sobredispersão também para as contagens dos totais de tentativas, além incluir covariâncias possivelmente diferentes entre as contagens em diversos instantes de avaliação. Neste novo modelo, introduzimos um parâmetro que relaciona o total de tentativas com a probabilidade de acerto, tornando-o ainda mais geral. Obtemos estimadores de máxima verossimilhança dos parâmetros utilizando um algoritmo de Newton-Raphson. Consideramos um outro conjunto de dados provenientes do mesmo estudo para ilustração da metodologia proposta.

Palavras-chave: contagens bivariadas, dados longitudinais, sobredispersão, efeitos aleatórios, modelos de regressão. 


\section{Abstract}

In Lora and Singer (Statistics in Medicine, 2008), we proposed a Beta-Binomial/ Poisson p-variate model to analyze data from a study which consists in counting the number of trials and successes of a manual exercise in one minute periods, done by Parkinson's disease patients, before and after a training. The purpose was to verify if the training im-

proves the number of trials and the percentage of success, which emphasizes the bivariate aspect of the problem. This model considers these characteristics, uses an adequate distribution to count data and settles the overdispersion suggested in the number os successes. As a generalization, initially, we propose a Beta-Binomial/Poisson-Gama model which also settles the overdispersion suggested by the total number of trials, besides includes possible different covariances between total trial counts in different evaluation instants. In this new model, we introduce a parameter that links the total trials with the success probability, making it even more general. We obtain maximum likelihood estimators for the parameters using an Newton-Raphson algorithm. We consider another data from the same study to illustrate the proposal methodology.

Key-words: bivariate counts, longitudinal data, overdispersion, random effects, regression models 


\section{Sumário}

1 Introdução $\quad 2$

1.1 Exemplo . . . . . . . . . . . . . . . . . . 2

1.2 Análise do exemplo por meio do modelo Beta-Binomial/Poisson p-variado . 8

1.3 Propostas . . . . . . . . . . . . . . . . . . . 16

2 Modelo Beta-Binomial/Poisson-Gama 18

2.1 Modelo . . . . . . . . . . . . . . . . . . . . . . 18

2.2 Análise do exemplo por meio do modelo Beta-Binomial/Poisson-Gama . 22

3 Modelos Beta-Binomial/Poisson-Gama com enlace 28

3.1 Modelo Beta-Binomial/Poisson-Gama com enlace gerado pelas probabilidades esperadas de sucesso . . . . . . . . . . . . . . . . . . . 29

3.2 Análise do exemplo por meio do modelo Beta-Binomial/Poisson-Gama com enlace gerado pelas probabilidades esperadas de sucesso . . . . . . . . . . 30

3.3 Modelo Beta-Binomial/Poisson-Gama com enlace gerado pelas probabilidades individuais de sucesso . . . . . . . . . . . . . . . . . . . . . 39

3.4 Análise do exemplo por meio do modelo Beta-Binomial/Poisson-Gama com enlace gerado pelas probabilidades individuais de sucesso . . . . . . . . . . 42

3.5 Aspectos computacionais . . . . . . . . . . . . . . . . . 44

$\begin{array}{lll}4 & \text { Discussão } & 48\end{array}$

$\begin{array}{ll}\text { A Correlações amostrais } & 50\end{array}$

B Derivadas sob o modelo Poisson-Gama 53 
C Derivadas sob o modelo Beta-Binomial/Poisson-Gama com enlace gerado pelas probabilidades esperadas de sucesso

D Derivadas sob o modelo Beta-Binomial/Poisson-Gama com enlace gerado pelas probabilidades individuais de sucesso

Referências Bibliográficas 


\section{Capítulo 1}

\section{Introdução}

Modelos Beta-Binomial/Poisson têm sido usados para analisar dados distribuídos segundo uma distribuição Binomial com sobredispersão, quando o número de ensaios é considerado aleatório. Rai e van Ryzin (1985), Dunson (1973), e Allen e Barnhart (2002), por exemplo, usaram tais modelos para estudos de dose-resposta. Distribuições de Poisson bivariadas também foram usadas por Comulada e Weiss (2007), e por Karli e Ntzoufras (2003) para analisar dados de contagens correlacionados. Por outro lado, Nelson (1985), por exemplo, usou modelos Poisson-Gama para analisar contagens longitudinais. Zhu et al. (2003) acrescentaram um parâmetro que relaciona o total de tentativas com a probabilidade de acerto a esses modelos Beta-Binomial/Poisson. Lora (2004) e Lora e Singer (2008) estenderam tais modelos para medidas repetidas, com covariáveis, usando distribuições de Poisson multivariadas propostas por Holgate (1964) e empregadas em problemas de regressão por Ho e Singer $(1997,2001)$. Ilustramos a análise baseada nesse modelo por meio do seguinte exemplo.

\subsection{Exemplo}

A doença de Parkinson é uma das patologias degenerativas mais freqüentes do sistema nervoso central, caracterizando-se principalmente por sintomas motores, tendo o tremor de repouso como sintoma inicial na maioria dos casos, e a bradicinesia (dificuldade global para iniciar movimentos), como limitante das atividades cotidianas do paciente. O 
início da doença ocorre em geral entre 50 e 70 anos, independentemente do gênero. Neste contexto, o objetivo de um estudo realizado no Laboratório de Aprendizado Processual do Departamento de Fisioterapia, Fonoaudiologia e Terapia Ocupacional da Faculdade de Medicina da Universidade de São Paulo é verificar se o treinamento de atividades motoras pode interferir na bradicinesia, melhorando as condições motoras dos doentes. Foram selecionados 25 pacientes com diagnóstico confirmado de doença de Parkinson (grupo experimental) e um grupo controle de 21 pessoas sem antecedentes de alterações neurológicas detectáveis. Os pacientes do grupo experimental foram classificados de acordo com o estágio da doença em duas categorias (estágio inicial e estágio avançado). Para mais detalhes, ver Singer et al. (2002) e Piemonte (2003).

O estudo envolveu a repetição de duas sequências predeterminadas de oposição dos dedos (toques do polegar em um dos outros quatro dedos, numerados de 1 a 4), antes e depois de um treinamento. Durante a execução das sequências, foi registrado o número daquelas realizadas correta e incorretamente, por intermédio de uma luva conectada a um computador. Na avaliação inicial, os participantes realizaram cada uma das sequências durante um minuto, com as duas mãos. Após essa avaliação inicial, todos os participantes realizaram um treinamento de quatro semanas, praticando apenas uma das sequências com uma das mãos. Tanto no grupo controle como no experimental, metade dos participantes realizou o treinamento com a mão preferencial e a outra metade, com a mão não preferencial, formando quatro subgrupos. Para o grupo controle, mão preferencial é a esquerda para canhotos e a direita para destros; para o grupo experimental, mão preferencial é a menos afetada pela doença. Metade dos participantes de cada um dos quatro subgrupos iniciais treinou uma das sequências e a outra metade treinou a outra, gerando oito subgrupos. Após o treinamento, os participantes foram reavaliados da mesma maneira que no instante inicial (realizando as duas sequências por um minuto com as duas mãos) e também realizando as mesmas sequências sob condições dificultadoras (por exemplo, sem indicação visual dos números correspondentes aos dedos na luva). Foram registrados os números de sequências corretas e incorretas.

Problemas técnicos impediram o registro de contagens para a mão não treinada dos participantes. Desta maneira, para cada participante obtiveram-se as contagens para 
a mão treinada, com as duas sequências (treinada e controle) em três condições: antes e depois do treinamento e sob as condições dificultadoras.

Na Tabela 1.1 estão apresentadas as médias e variâncias das contagens de acertos e totais para as sequências treinada e controle tanto na avaliação final quanto sob a condição dificultadora, para seis subgrupos, formados pelas combinações de estágio da doença (controle, estágio inicial e avançado) e uso da mão preferencial (sim ou não). Os dados evidenciaram sobredispersão, no sentido referido por Nelder e McCullagh (1989), por exemplo. Além disso, na Tabela 1.2 estão apresentados os coeficientes de correlação entre as respostas dos indivíduos do grupo controle, usando a mão preferencial, nas avaliações final e sob a condição dificultadora. As correlações entre as respostas dos indivíduos dos outros grupos são similares e estão apresentadas no Apêndice A.

Os objetivos desse estudo são verificar se o treinamento melhora o desempenho dos participantes na realização das sequências, verificar se essa possível melhora depende do estágio da doença (controles, doentes em estágio inicial e doentes em estágio avançado), e comparar a avaliação final com aquelas realizadas sob as condições dificultadoras. Para tanto, pretende-se avaliar se o treinamento tem efeito no número de tentativas por minuto (agilidade) e na porcentagem de tentativas certas (habilidade), o que destaca a característica bivariada do estudo. Além disso, há interesse também em comparar o desempenho da mão preferencial com aquele da mão não preferencial.

Para analisar os dados das avaliações inicial e final, Lora e Singer (2008) propõem um modelo Beta-Binomial/Poisson p-variado que considera simultaneamente o aspecto bivariado do estudo e a possível sobredispersão sugerida pela análise descritiva.

Seja

$$
\mathbf{Y}_{g}=\left(X_{g 1}, N_{g 1}, \ldots, X_{g p}, N_{g p}\right)^{\prime}
$$

o vetor de respostas para o $g$-ésimo indivíduo $(g=1, \ldots, m)$ com $X_{g h}$ correspondendo ao número de acertos em $N_{g h}$ tentativas na $h$-ésima $(h=1, \ldots, p)$ observação. Assumimos que, para todo $g$ e $h$, 
Tabela 1.1: Médias e variâncias para o número de acertos e total de tentativas dos grupos de estudo nas diferentes situações de realização das sequências

\begin{tabular}{|c|c|c|c|c|c|}
\hline Estágio & Avaliação & Mão & Sequência & Acertos (Variância) & Totais (Variância) \\
\hline controle & final & preferencial & treinada & $32,7(139,2)$ & $33,1(132,3)$ \\
\hline controle & final & preferencial & controle & $20,9(90,3)$ & $26,1(44,9)$ \\
\hline controle & final & não & treinada & $32,8(74,0)$ & $34,4(72,3)$ \\
\hline controle & final & não & controle & $24,2(25,0)$ & $28,6(38,4)$ \\
\hline controle & dificultadora & preferencial & treinada & $29,9(132,3)$ & $30,0(136,9)$ \\
\hline controle & dificultadora & preferencial & controle & $19,4(112,4)$ & $20,9(75,7)$ \\
\hline controle & dificultadora & não & treinada & $31,5(77,4)$ & $34,3(88,4)$ \\
\hline controle & dificultadora & não & controle & $19,0(50,4)$ & $24,1(20,3)$ \\
\hline inicial & final & preferencial & treinada & $20,2(9,6)$ & $21,8(2,9)$ \\
\hline inicial & final & preferencial & controle & $13,2(30,3)$ & $16,8(43,6)$ \\
\hline inicial & final & não & treinada & $20,1(33,6)$ & $20,4(39,7)$ \\
\hline inicial & final & não & controle & $15,3(112,4)$ & $20,3(116,6)$ \\
\hline inicial & dificultadora & preferencial & treinada & $13,2(70,6)$ & $18,8(43,6)$ \\
\hline inicial & dificultadora & preferencial & controle & $10,0(94,1)$ & $18,5(50,4)$ \\
\hline inicial & dificultadora & não & treinada & $17,4(380,3)$ & $20,7(324,0)$ \\
\hline inicial & dificultadora & não & controle & $9,1(104,0)$ & $15,3(82,8)$ \\
\hline avançado & final & preferencial & treinada & $13,5(90,3)$ & $14,9(77,4)$ \\
\hline avançado & final & preferencial & controle & $7,4(75,7)$ & $11,9(67,2)$ \\
\hline avançado & final & não & treinada & $22,5(75,7)$ & $23,8(75,7)$ \\
\hline avançado & final & não & controle & $5,8(31,4)$ & $12,8(12,3)$ \\
\hline avançado & dificultadora & preferencial & treinada & $11,4(187,7)$ & $15,8(187,7)$ \\
\hline avançado & dificultadora & preferencial & controle & $6,3(74,0)$ & $11,4(90,3)$ \\
\hline avançado & dificultadora & não & treinada & $14,0(20,3)$ & $19,3(34,8)$ \\
\hline avançado & dificultadora & não & controle & $8,8(5,8)$ & $17,5(43,6)$ \\
\hline
\end{tabular}


Tabela 1.2: Coeficientes de correlação entre as contagens na avaliação final e naquela realizada sob a condição dificultadora para participantes do grupo controle usando a mão preferencial

\begin{tabular}{|c|c|c|c|c|c|c|c|c|c|c|}
\hline & & & \multicolumn{4}{|c|}{ Avaliação final } & \multicolumn{4}{|c|}{ Condição Dificultadora } \\
\hline & & & \multicolumn{2}{|c|}{ Seq. treinada } & \multicolumn{2}{|c|}{ Seq. controle } & \multicolumn{2}{|c|}{ Seq. treinada } & \multicolumn{2}{|c|}{ Seq. controle } \\
\hline & & & Suc. & Tent. & Suc. & Tent. & Suc. & Tent. & Suc. & Tent. \\
\hline \multirow{4}{*}{$\begin{array}{l}\text { Avaliação } \\
\text { final }\end{array}$} & Seq. & Suc. & 1 & & & & & & & \\
\hline & trein. & Tent. & 0,99 & 1 & & & & & & \\
\hline & Seq. & Suc. & 0,60 & 0,61 & 1 & & & & & \\
\hline & controle & Tent. & 0,93 & 0,92 & 0,50 & 1 & & & & \\
\hline Condição & Seq. & Suc. & 0,90 & 0,87 & 0,59 & 0,84 & 1 & & & \\
\hline \multirow[t]{3}{*}{ Dificultadora } & trein. & Tent. & 0,89 & 0,86 & 0,59 & 0,82 & 0,99 & 1 & & \\
\hline & Seq. & Suc. & 0,55 & 0,56 & 0,82 & 0,57 & 0,68 & 0,67 & 1 & \\
\hline & controle & Tent. & 0,63 & 0,63 & 0,77 & 0,68 & 0,74 & 0,73 & 0,98 & 1 \\
\hline
\end{tabular}

Códigos: Suc.: Sucessos, Tent.: Tentativas e Seq.: Sequência

$$
\begin{aligned}
& X_{g h} \mid N_{g h}, \pi_{g h} \sim \operatorname{Binomial}\left(N_{g h}, \pi_{g h}\right) \text { independentes } \\
& \pi_{g h} \sim \operatorname{Beta}\left(\mu\left(\mathbf{z}_{\mu g h}\right) / \theta\left(\mathbf{z}_{\theta g h}\right),\left[1-\mu\left(\mathbf{z}_{\mu g h}\right)\right] / \theta\left(\mathbf{z}_{\theta g h}\right)\right) \text { independentes } \\
& \mathbf{N}_{g}=\left(N_{g 1}, \ldots, N_{g p}\right)^{\prime} \sim \operatorname{Poisson} \mathrm{p}-\operatorname{variada}\left(\lambda_{1}\left(\mathbf{z}_{\lambda g 1}\right), \ldots, \lambda_{p}\left(\mathbf{z}_{\lambda g p}\right), \lambda\left(\mathbf{z}_{\lambda g}\right)\right) \\
& \text { independentes }
\end{aligned}
$$

em que $\mathbf{z}_{\mu g h}, \mathbf{z}_{\theta g h}, \mathbf{z}_{\lambda g h}$ e $\mathbf{z}_{\lambda g}$ são vetores de covariáveis. Para nossos propósitos, as covariáveis correspondem às variáveis de classificação dos participantes (estágio da doença, uso da mão preferencial e sequência), mas poderiam corresponder a variáveis contínuas, como idade, tempo de diagnóstico etc.

Conforme (1.2) e (1.3), indivíduos do mesmo grupo não têm a mesma probabilidade de sucesso, mas essas probabilidades são geradas a partir de uma mesma distribuição. A parametrização $(0<\mu<1, \theta>0)$ usada em (1.3) foi sugerida por Gange et al. (1996) para facilitar a estimação via máxima verossimilhança; sua relação com a parametrização usual da distribuição $\operatorname{Beta}(a, b)$ (como em Johnson e Kotz (1970), por exemplo) é dada 
por

$$
\mu=\frac{a}{a+b} \quad \text { e } \quad \theta=\frac{1}{a+b} .
$$

A distribuição Poisson p-variada em (1.4) é uma generalização do caso bivariado apresentado por Holgate (1964).

O primeiro e segundo momentos centrais de $\pi_{g h}$ em (1.3) são

$$
\begin{aligned}
& E\left(\pi_{g h}\right)=\mu\left(\mathbf{z}_{\mu g h}\right) \\
& \operatorname{Var}\left(\pi_{g h}\right)=\mu\left(\mathbf{z}_{\mu g h}\right)\left[1-\mu\left(\mathbf{z}_{\mu g h}\right)\right] \theta\left(\mathbf{z}_{\theta g h}\right)\left[1+\theta\left(\mathbf{z}_{\theta g h}\right)\right]^{-1}
\end{aligned}
$$

Também, de (1.2) e (1.3), podemos concluir que, para todo $g$ e $h$,

$$
X_{g h} \mid N_{g h} \sim \text { Beta - Binomial }\left[N_{g h}, \mu\left(\mathbf{z}_{\mu g h}\right), \theta\left(\mathbf{z}_{\theta g h}\right)\right]
$$

com

$$
\begin{aligned}
& E\left(X_{g h} \mid N_{g h}\right)=N_{g h} \mu\left(\mathbf{z}_{\mu g h}\right) \\
& \operatorname{Var}\left(X_{g h} \mid N_{g h}\right)=N_{g h} \mu\left(\mathbf{z}_{\mu g h}\right)\left[1-\mu\left(\mathbf{z}_{\mu g h}\right)\right]\left[1+N_{g h} \theta\left(\mathbf{z}_{\theta g h}\right)\right]\left[1+\theta\left(\mathbf{z}_{\theta g h}\right)\right]^{-1}
\end{aligned}
$$

O primeiro e segundo momentos centrais para o número de sucessos são

$$
\begin{aligned}
& E\left(X_{g h}\right)=\lambda_{h}\left(\mathbf{z}_{\lambda g h}\right) \mu\left(\mathbf{z}_{\mu g h}\right) \\
& \operatorname{Var}\left(X_{g h}\right)=\lambda_{h}\left(\mathbf{z}_{\lambda g h}\right) \mu\left(\mathbf{z}_{\mu g h}\right)+\lambda_{h}^{2}\left(\mathbf{z}_{\lambda g h}\right) \mu\left(\mathbf{z}_{\mu g h}\right)\left[1-\mu\left(\mathbf{z}_{\mu g h}\right)\right] \frac{\theta\left(\mathbf{z}_{\theta g h}\right)}{1+\theta\left(\mathbf{z}_{\theta g h}\right)} \\
& \operatorname{Cov}\left(X_{g h}, X_{g h^{\prime}}\right)=\lambda\left(\mathbf{z}_{\lambda g}\right) \mu\left(\mathbf{z}_{\mu g h}\right) \mu\left(\mathbf{z}_{\mu g h^{\prime}}\right)
\end{aligned}
$$

para todo $g, h, h^{\prime}, h \neq h^{\prime}$.

Esses resultados evidenciam que o valor esperado do número de sucessos é função de dois componentes: o primeiro, $\lambda_{h}\left(\mathbf{z}_{\lambda g h}\right)$, relaciona-se à agilidade com que os indivíduos realizam as tentativas, e o segundo, $\mu\left(\mathbf{z}_{\mu g h}\right)$, à habilidade com que acertam as sequências pré-determinadas de movimentos.

Analogamente, o primeiro e segundo momento central do número de tentativas são

$$
\begin{aligned}
& E\left(N_{g h}\right)=\operatorname{Var}\left(N_{g h}\right)=\lambda_{h}\left(\mathbf{z}_{\lambda g h}\right) \\
& \operatorname{Cov}\left(N_{g h}, N_{g h^{\prime}}\right)=\lambda\left(\mathbf{z}_{\lambda g}\right)
\end{aligned}
$$


para todo $g, h, h^{\prime}, h \neq h^{\prime}$.

Os parâmetros $\theta\left(\mathbf{z}_{\theta g h}\right)$ afetam não só a variabilidade do número de sucessos, mas também a magnitude da sobredispersão correspondente. Quando $\theta\left(\mathbf{z}_{\theta g h}\right)=0$, não há sobredispersão e $X_{g h} \mid N_{g h} \sim \operatorname{Binomial}\left(N_{g h}, \pi_{g h}\right)$. Conforme $\theta\left(\mathbf{z}_{\theta g h}\right)$ aumenta, a sobredispersão também aumenta, tendendo para seu máximo, $N_{g h}^{2} \mu\left(\mathbf{z}_{\mu g h}\right)\left[1-\mu\left(\mathbf{z}_{\mu g h}\right)\right]$, quando $\theta\left(\mathbf{z}_{\theta g h}\right) \rightarrow \infty$

A covariância entre o número de sucessos e o número de tentativas é

$$
\operatorname{Cov}\left(X_{g h}, N_{g h}\right)=\lambda_{h}\left(\mathbf{z}_{\lambda g h}\right) \mu\left(\mathbf{z}_{\mu g h}\right)
$$

Para investigar os efeitos das covariáveis, adotamos modelos log-lineares da forma

$$
\begin{aligned}
& \mu\left(\mathbf{z}_{\mu g h}\right)=\frac{\exp \left(\mathbf{z}_{\mu g h}^{\prime} \boldsymbol{\beta}_{\mu}\right)}{1+\exp \left(\mathbf{z}_{\mu g h}^{\prime} \boldsymbol{\beta}_{\mu}\right)} \\
& \theta\left(\mathbf{z}_{\theta g h}\right)=\exp \left(\mathbf{z}_{\theta g h}^{\prime} \boldsymbol{\beta}_{\theta}\right) \\
& \lambda_{h}\left(\mathbf{z}_{\lambda g h}\right)=\exp \left(\mathbf{z}_{\lambda g h}^{\prime} \boldsymbol{\beta}_{\lambda h}\right), h=1, \ldots, p \\
& \lambda\left(\mathbf{z}_{\lambda g}\right)=\exp \left(\mathbf{z}_{\lambda g}^{\prime} \boldsymbol{\beta}_{\lambda}\right)
\end{aligned}
$$

em que $\boldsymbol{\beta}_{\mu}, \boldsymbol{\beta}_{\theta}, \boldsymbol{\beta}_{\lambda h}, h=1, \ldots, p$, e $\boldsymbol{\beta}_{\lambda}$ são vetores de parâmetros a serem estimados.

Para motivar os modelos propostos nos próximos capítulos, inicialmente utilizamos aquele proposto em Lora e Singer (2008) na análise dos dados da avaliação final e daquela obtida sob a condição dificultadora. Para estimar os vetores de parâmetros usamos processos iterativos de Newton-Raphson, cujos códigos implementados em R podem ser obtidos em http://www. ime.usp.br/ jmsinger.

\subsection{Análise do exemplo por meio do modelo Beta- Binomial/Poisson p-variado}

Como estratégia de análise, inicialmente ajustamos modelos log-lineares com todos os fatores principais e interações de primeira ordem, e depois tentamos reduzi-los

eliminando os termos não significantes por meio de testes da razão de verossimilhanças. Os parâmetros foram indexados pelo estágio da doença $(0=$ controle, $1=$ inicial, 2=avançado), mão utilizada $(\mathrm{P}=$ preferencial, $\mathrm{N}=$ não preferencial), avaliação ( $\mathrm{F}=$ final, 
$\mathrm{D}=$ sob a condição dificultadora) e sequência $(\mathrm{T}=$ treinada, $\mathrm{C}=$ controle). Adotamos uma parametrização de cela de referência, focada nos participantes do grupo controle (0) realizando a sequência treinada $(\mathrm{T})$ usando a mão preferencial $(\mathrm{P})$ na avaliação final $(\mathrm{F})$.

Sob o modelo inicial, o vetor de parâmetros para o número de acertos é $\boldsymbol{\beta}=\left(\boldsymbol{\beta}_{\mu}, \boldsymbol{\beta}_{\theta}\right)$ em que

$$
\begin{aligned}
\boldsymbol{\beta}_{m}= & \left(\beta_{m 0}, \beta_{m 1}, \beta_{m 2}, \beta_{m N}, \beta_{m D}, \beta_{m C},\right. \\
& \left.\beta_{m(1 * D)}, \beta_{m(1 * N)}, \beta_{m(1 * C)}, \beta_{m(2 * D)}, \beta_{m(2 * N)}, \beta_{m(2 * C)}, \beta_{m(D * N)}, \beta_{m(D * C)}, \beta_{m(N * C)}\right),
\end{aligned}
$$

$\operatorname{com} m=\mu, \theta$.

Para a probabilidade esperada de acertos, por exemplo, $\beta_{\mu 0}$ corresponde ao logaritmo da chance de indivíduos controle, usando a mão preferencial, realizando a sequência controle durante a avaliação final; $\beta_{\mu N}$ corresponde ao acréscimo no logaritmo da chance devido ao efeito do uso da mão não preferencial em relação ao uso da preferencial; e $\beta_{\mu(1 * N)}$ corresponde à interação entre o estágio inicial da doença (1) e o uso da mão não preferencial $(N)$.

O processo iterativo proposto foi sensível aos valores iniciais, especialmente àqueles relacionados às interações, divergindo quando muitos parâmetros são introduzidos simultaneamente. Para superar tal dificuldade, começamos com um modelo mais simples contendo apenas os efeitos principais e usamos as estimativas obtidas como valores iniciais para ajustar outros modelos, que incluem as interações. Nesse processo, estimativas obtidas por meio do método dos momentos foram usadas como valores iniciais, tanto para os efeitos principais, como para as interações. Nos casos em que o processo não convergiu, o valor inicial das interações foi substituído por zero.

As interações não significativas foram identificadas e sua eliminação do modelo inicial foi justificada pelo resultado $(p=0,258)$ do teste da hipótese

$$
\begin{aligned}
H_{0}: & \beta_{\mu(1 * D)}, \beta_{\mu(1 * N)}, \beta_{\mu(1 * C)}, \beta_{\mu(2 * D)}, \beta_{\mu(2 * N)}, \beta_{\mu(2 * C)}, \beta_{\mu(D * N)}, \beta_{\mu(D * C)}, \beta_{\mu(N * C)}, \\
& \beta_{\theta(1 * D)}, \beta_{\theta(1 * C)}, \beta_{\theta(2 * N)}, \beta_{\theta(2 * C)}, \beta_{\theta(D * N)}, \beta_{\theta(D * C)}, \beta_{\theta(N * C)}=0 .
\end{aligned}
$$

Sob o modelo reduzido, os efeitos principais não significativos foram identificados e sua eliminação foi justificada pelo resultado $(p=0,200)$ do teste da hipótese

$$
H_{0}: \beta_{\mu N}, \beta_{\theta 1}, \beta_{\theta C}=0
$$


Testamos a eliminação de efeitos principais mesmo na presença de interações relacionadas a eles pois, por exemplo, caso eliminássemos o efeito principal $\beta_{\mu C}$ na presença da interação $\beta_{\mu(D * C)}$, isso significaria que não há diferença entre a probabilidade de sucesso para as sequências treinada e controle na avaliação final, mas há diferença entre tais probabilidades sob a condição dificultadora. A qualidade de ajuste do modelo final pode ser justificada por um teste da razão de verossimilhanças comparando-o com o modelo inicial ( $p=$ $0,202)$.

As estimativas dos parâmetros desse modelo final (com respectivos erros padrões) estão apresentadas na Tabela 1.3. Estimativas do valor esperado da probabilidade de acerto $\left[E\left(\pi_{g h}\right)=\mu\left(\mathbf{z}_{\mu g h}\right)\right]$ sob o modelo final (com respectivos erros padrões) estão apresentadas na Tabela 1.4. Estimativas dos parâmetros de dispersão $\theta\left(\mathbf{z}_{\theta g h}\right)$, relacionados à variabilidade da probabilidade de acerto, com respectivos erros padrões, estão apresentadas na Tabela 1.5.

Tabela 1.3: Estimativas dos parâmetros do modelo Beta-Binomial final e erros padrões

\begin{tabular}{llcc}
\hline Parâmero & Relacionado a & Estimativa & Erro padrão \\
\hline$\beta_{\mu 0}$ & grupo controle, mão preferencial & 3,16 & 0,23 \\
& avaliação final e sequência treinada & & \\
$\beta_{\mu 1}$ & efeito do estágio inicial & $-1,07$ & 0,26 \\
$\beta_{\mu 2}$ & efeito do estágio avançado & $-1,59$ & 0,25 \\
$\beta_{\mu D}$ & efeito da condição dificultadora & $-0,77$ & 0,22 \\
$\beta_{\mu C}$ & efeito da sequência controle & $-1,28$ & 0,21 \\
\hline$\beta_{\theta 0}$ & grupo controle, mão preferencial & $-0,76$ & 0,32 \\
& avaliação final e sequência treinada & & \\
$\beta_{\theta 2}$ & efeito do estágio avançado & 1,31 & 0,49 \\
$\beta_{\theta D}$ & efeito da condição dificultadora & 1,26 & 0,36 \\
$\beta_{\theta N}$ & efeito da mão não preferencial & $-1,91$ & 0,33 \\
$\beta_{\theta(1 * N)}$ & efeito do estágio inicial e mão não preferencial & 1,66 & 0,46 \\
$\beta_{\theta(2 * D)}$ & efeito do estágio avançado e condição dificultadora & $-1,89$ & 0,66 \\
\hline
\end{tabular}

Os resultados sugerem que não há diferença entre os valores esperados da probabilidade de sucesso para participantes que utilizam ou não utilizam a mão preferencial $\left(\beta_{\mu N}=0\right)$. Pacientes em estágio inicial da doença têm probabilidade esperada de acerto 
Tabela 1.4: Estimativas das probabilidades esperadas de sucesso e erros padrões

\begin{tabular}{cccccc}
\hline $\begin{array}{c}\text { Estágio } \\
\text { da doença }\end{array}$ & Avaliação & Mão & Sequência & $\begin{array}{c}\text { Valor } \\
\text { esperado }\end{array}$ & $\begin{array}{c}\text { Erro } \\
\text { padrão }\end{array}$ \\
\hline controle & final & ambas & treinada & 0,96 & 0,01 \\
controle & final & ambas & controle & 0,87 & 0,02 \\
controle & dificultadora & ambas & treinada & 0,92 & 0,02 \\
controle & dificultadora & ambas & controle & 0,75 & 0,04 \\
\hline inicial & final & ambas & treinada & 0,89 & 0,03 \\
inicial & final & ambas & controle & 0,69 & 0,05 \\
inicial & dificultadora & ambas & treinada & 0,79 & 0,04 \\
inicial & dificultadora & ambas & controle & 0,51 & 0,06 \\
\hline avançado & final & ambas & treinada & 0,83 & 0,04 \\
avançado & final & ambas & controle & 0,57 & 0,06 \\
avançado & dificultadora & ambas & treinada & 0,69 & 0,05 \\
avançado & dificultadora & ambas & controle & 0,38 & 0,06 \\
\hline
\end{tabular}

menor que os controles, e aqueles em estágio avançando têm probabilidade de sucesso esperada ainda menor $\left(\beta_{\mu 2}<\beta_{\mu 1}<0\right)$. Além disso, a probabilidade esperada de acerto na realização da sequência controle é menor do que a probabilidade esperada de acerto na realização da sequência treinada $\left(\beta_{\mu C}<0\right)$. Por fim, a probabilidade esperada de sucesso sob a condição dificultadora é menor do que a probabilidade esperada de sucesso na avaliação final $\left(\beta_{\mu D}<0\right)$.

Podemos concluir também que não há diferença nem entre os valores esperados dos parâmetros de dispersão para participantes do grupo controle e para pacientes em estágio inicial da doença $\left(\beta_{\theta 1}=0\right)$, nem para as respostas obtidas sob as sequências treinada e controle $\left(\beta_{\theta C}=0\right)$. Valores esperados do parâmetro de dispersão são maiores para pacientes em estágio avançado do que para participantes controle e em estágio inicial $\left(\beta_{\theta 2}>0\right)$. As respostas obtidas sob a condição dificultadora também apresentam dispersão esperada maior do que aquelas obtidas na avaliação final $\left(\beta_{\theta D}>0\right)$. Participantes que utilizam a mão não preferencial apresentam valor esperado menor do que aqueles que a utilizam $\left(\beta_{\theta N}<0\right)$. Além disso, tais valores aumentam quando se trata de participantes em estágio inicial da doença usando a mão não preferencial $\left(\beta_{\theta(1 * N)}>0\right)$, e diminuem 
Tabela 1.5: Estimativas dos valores esperados dos parâmetros de dispersão e erros padrões

\begin{tabular}{cccccc}
\hline $\begin{array}{c}\text { Estágio } \\
\text { da doença }\end{array}$ & Avaliação & Mão & Sequência & $\begin{array}{c}\text { Valor } \\
\text { esperado }\end{array}$ & $\begin{array}{c}\text { Erro } \\
\text { padrão }\end{array}$ \\
\hline controle & final & preferencial & ambas & 0,47 & 0,15 \\
controle & final & não & ambas & 0,07 & 0,02 \\
controle & dificultadora & preferencial & ambas & 1,63 & 0,48 \\
controle & dificultadora & não & ambas & 0,24 & 0,08 \\
\hline inicial & final & preferencial & ambas & 0,47 & 0,15 \\
inicial & final & não & ambas & 0,36 & 0,16 \\
inicial & dificultadora & preferencial & ambas & 1,63 & 0,48 \\
inicial & dificultadora & não & ambas & 1,27 & 0,48 \\
\hline avançado & final & preferencial & ambas & 1,72 & 0,74 \\
avançado & final & não & ambas & 0,26 & 0,12 \\
avançado & dificultadora & preferencial & ambas & 0,92 & 0,37 \\
avançado & dificultadora & não & ambas & 0,14 & 0,07 \\
\hline
\end{tabular}

quando se trata de indivíduos em estágio avançado da doença sob a condição dificultadora $\left(\beta_{\theta(2 * D)}<0\right)$.

No modelo Poisson $p$-variado para o total de tentativas, os $p=4$ instantes de observação correspondem à combinação dos níveis de sequência (treinada ou controle) e instante de avaliação (final ou sob a condição dificultadora), e o índice $h$ assume os valores $F T, F C, D T$ e $D C$. Os componentes do vetor de parâmetros $\boldsymbol{\beta}=\left(\boldsymbol{\beta}_{\lambda F T}, \boldsymbol{\beta}_{\lambda F C}, \boldsymbol{\beta}_{\lambda D T}\right.$, $\boldsymbol{\beta}_{\lambda D C}, \boldsymbol{\beta}_{\lambda}$ ) para a cela de referência [grupo controle (0) usando a mão preferencial (P)] são

$$
\begin{aligned}
\boldsymbol{\beta}_{\lambda h} & =\left(\beta_{\lambda h 0}, \beta_{\lambda h 1}, \beta_{\lambda h 2}, \beta_{\lambda h N}, \beta_{\lambda h(1 * N)}, \beta_{\lambda h(2 * N)}\right), h=F T, F C, D T, D C, \\
\boldsymbol{\beta}_{\lambda} & =\left(\beta_{\lambda 0}, \beta_{\lambda 1}, \beta_{\lambda 2}, \beta_{\lambda N}, \beta_{\lambda(1 * N)}, \beta_{\lambda(2 * N)}\right) .
\end{aligned}
$$

As interações não significantes foram identificadas e sua eliminação do modelo inicial pode ser justificada pelo resultado $(p=0,657)$ do teste da hipótese

$$
H_{0}: \quad \beta_{\lambda F T(1 * N)}, \beta_{\lambda F C(1 * N)}, \beta_{\lambda F C(2 * N)}, \beta_{\lambda D T(1 * N)}, \beta_{\lambda D T(2 * N)}, \beta_{\lambda D C(2 * N)}, \beta_{\lambda(1 * N)}, \beta_{\lambda(2 * N)}=0
$$

Sob o modelo reduzido, os efeitos principais não significativos foram identificados e sua 
eliminação pode ser justificada pelo resultado $(p=0,415)$ do teste da hipótese

$$
H_{0}: \beta_{\lambda F C N}, \beta_{\lambda D C 1}, \beta_{\lambda 1}, \beta_{\lambda N}=0
$$

O ajuste do modelo reduzido pode ser justificado pelo teste da razão de verossimilhanças, comparando-o com o modelo inicial $(p=0,617)$. As estimativas dos parâmetros (com seus respectivos erros padrões) estão apresentadas na Tabela 1.6.

Tabela 1.6: Estimativas dos parâmetros e erros padrões para o modelo Poisson reduzido

\begin{tabular}{llcc}
\hline Parâmetro & Relacionado a & Estimativa & Erro padrão \\
\hline$\beta_{\lambda F T 0}$ & grupo controle, mão preferencial & 3,51 & 0,04 \\
$\beta_{\lambda F T 1}$ & efeito do estágio inicial & $-0,45$ & 0,07 \\
$\beta_{\lambda F T 2}$ & efeito do estágio avançado & $-0,79$ & 0,09 \\
$\beta_{\lambda F T(1 * N)}$ & efeito do estágio inicial e mão não preferencial & 0,41 & 0,13 \\
\hline$\beta_{\lambda F C 0}$ & grupo controle, mão preferencial & 3,30 & 0,04 \\
$\beta_{\lambda F C 1}$ & efeito do estágio inicial & $-0,36$ & 0,07 \\
$\beta_{\lambda F C 2}$ & efeito do estágio avançado & $-0,80$ & 0,09 \\
\hline$\beta_{\lambda D T 0}$ & grupo controle, mão preferencial & 3,40 & 0,05 \\
$\beta_{\lambda D T 1}$ & efeito do estágio inicial & $-0,46$ & 0,07 \\
$\beta_{\lambda D T 2}$ & efeito do estágio avançado & $-0,61$ & 0,08 \\
$\beta_{\lambda D T N}$ & efeito da mão não preferencial & 0,11 & 0,05 \\
\hline$\beta_{\lambda D C 0}$ & grupo controle, mão preferencial & 2,99 & 0,05 \\
$\beta_{\lambda D C 2}$ & efeito do estágio avançado & $-0,46$ & 0,09 \\
$\beta_{\lambda D C N}$ & efeito da mão não preferencial & 0,20 & 0,07 \\
$\beta_{\lambda D C(1 * N)}$ & efeito do estágio inicial e mão não preferencial & $-0,46$ & 0,10 \\
\hline$\beta_{\lambda 0}$ & grupo controle, mão preferencial & 2,22 & 0,12 \\
$\beta_{\lambda 2}$ & efeito do estágio avançado & $-0,63$ & 0,22 \\
\hline & & & \\
\hline
\end{tabular}

Para comparar os diferentes instantes de avaliação, sob o modelo reduzido, consideramos inicialmente a hipótese

$$
\begin{aligned}
H_{0}: & \beta_{\lambda F T 1}=\beta_{\lambda F C 1}=\beta_{\lambda D T 1}, \\
\beta_{\lambda F T 2} & =\beta_{\lambda F C 2}=\beta_{\lambda D T 2}, \\
\beta_{\lambda D T N} & =\beta_{\lambda D C N}
\end{aligned}
$$


que corresponde à igualdade dos efeitos dos estágios da doença para as duas sequências realizadas na avaliação final, à igualdade dos efeitos dos estágios da doença sob a condição dificultadora realizando a sequência treinada, e à igualdade do efeito da utilização da mão não preferencial nas duas sequências sob a condição dificultadora.

Essa hipótese foi rejeitada $(p=0,002)$ por intermédio de um teste da razão de verossimilhanças. Sub-hipóteses de (1.19) foram testadas mas não confirmadas $(p<0,05)$. Assim a partir do modelo reduzido apresentado na Tabela 1.6, foram calculados os valores esperados dos totais de tentativas (Tabela 1.7) e das covariâncias entre as respostas obtidas sob as quatro condições de avaliação (Tabela 1.8).

Tabela 1.7: Estimativas dos valores esperados dos totais de tentativas

\begin{tabular}{cccccc}
\hline $\begin{array}{c}\text { Estágio } \\
\text { da doença }\end{array}$ & Avaliação & Mão & Sequência & $\begin{array}{c}\text { Valor } \\
\text { esperado }\end{array}$ & $\begin{array}{c}\text { Erro } \\
\text { padrão }\end{array}$ \\
\hline controle & final & ambas & treinada & 33,6 & 1,2 \\
controle & final & ambas & controle & 27,2 & 1,1 \\
controle & dificultadora & preferencial & treinada & 30,2 & 1,5 \\
controle & dificultadora & preferencial & controle & 19,8 & 1,0 \\
controle & dificultadora & não & treinada & 33,6 & 1,5 \\
controle & dificultadora & não & controle & 24,2 & 1,3 \\
\hline inicial & final & preferencial & treinada & 21,4 & 1,2 \\
inicial & final & ambas & controle & 19,1 & 1,1 \\
inicial & final & não & treinada & 32,4 & 4,5 \\
inicial & dificultadora & preferencial & treinada & 19,1 & 1,3 \\
inicial & dificultadora & preferencial & controle & 19,8 & 1,0 \\
inicial & dificultadora & não & treinada & 21,3 & 1,3 \\
inicial & dificultadora & não & controle & 15,3 & 1,3 \\
\hline avançado & final & ambas & treinada & 15,3 & 1,3 \\
avançado & final & ambas & controle & 12,2 & 1,0 \\
avançado & dificultadora & preferencial & treinada & 16,3 & 1,2 \\
avançado & dificultadora & preferencial & controle & 12,5 & 1,0 \\
avançado & dificultadora & não & treinada & 18,2 & 1,4 \\
avançado & dificultadora & não & controle & 15,3 & 1,4 \\
\hline
\end{tabular}


Tabela 1.8: Estimativas das covariâncias entre os totais de tentativas

\begin{tabular}{cccc}
$\begin{array}{c}\text { Estágio } \\
\text { da doença }\end{array}$ & Mão & $\begin{array}{c}\text { Valor } \\
\text { esperado }\end{array}$ & $\begin{array}{c}\text { Erro } \\
\text { padrão }\end{array}$ \\
\hline controle ou inicial & ambas & 9,2 & 1,1 \\
avançado & ambas & 4,4 & 0,9 \\
\hline
\end{tabular}

Podemos concluir que não há diferença entre os valores esperados dos totais de tentativas para participantes que usam ou não a mão preferencial na avaliação final realizando a sequência controle $\left(\beta_{\lambda F C N}=0\right)$. Apenas para os pacientes em estágio inicial da doença na avaliação final realizando a sequência treinada pode-se afirmar que os que usam a mão não preferencial apresentam valor esperado do total de tentativas maior do que aqueles que não a usam $\left(\beta_{\lambda F T(1 * N)}>0\right)$. Sob a condição dificultadora, para ambas as sequências, o valor esperado do total de tentativas para participantes usando a mão não preferencial é maior do que o valor esperado do total de tentativas de participantes que a usam $\left(\beta_{\lambda D T N}>0\right.$ e $\left.\beta_{\lambda D C N}>0\right)$, porém tal relação se inverte quando se trata dos pacientes em estágio inicial da doença, realizando a sequência controle $\left(\beta_{\lambda D C N}+\beta_{\lambda D C(1 * N)}<0\right)$. Por fim, nota-se que pacientes em estágio inicial da doença estão associados com valor esperado do total de tentativas menor do que os participantes controle, e aqueles em estágio avançado, com um valor esperado ainda menor para ambas as sequências da avaliação final e para a sequência treinada sob a condição dificultadora $\left(\beta_{\lambda F T 2}<\beta_{\lambda F T 1}<\right.$ $0, \beta_{\lambda F C 2}<\beta_{\lambda F C 1}<0$ e $\left.\beta_{\lambda D T 2}<\beta_{\lambda D T 1}<0\right)$. Para a sequência controle sob a condição dificultadora não há diferença entre o valor esperado do total de tentativas dos grupos controle e em estágio inicial da doença $\left(\beta_{\lambda D C 1}=0\right)$, mas os pacientes em estágio avançado da doença apresentam valor esperado menor $\left(\beta_{\lambda D C 2}<0\right)$.

Podemos também concluir que a covariância esperada entre os totais de tentativas para os 4 instantes de observação são iguais para participantes usando ou não a mão preferencial $\left(\beta_{\lambda N}=0\right)$. Além disso, a covariância esperada entre os totais de tentativas para todas as observações são iguais para participantes do grupo controle e em estágio inicial da doença $\left(\beta_{\lambda 1}=0\right)$, e esta covariância é maior do que aquela correspondente aos participantes em estágio avançado da doença $\left(\beta_{\lambda 2}<0\right)$. 
Tabela 1.9: Estimativas dos valores esperados dos acertos e totais de tentativas, e respectivos erros padrões (entre parênteses)

\begin{tabular}{cccccc}
\hline $\begin{array}{c}\text { Estágio } \\
\text { da doença }\end{array}$ & Avaliação & Mão & Sequência & Acertos & Totais \\
\hline controle & final & ambas & treinada & $32,3(1,2)$ & $33,6(1,2)$ \\
controle & final & ambas & controle & $23,7(1,1)$ & $27,2(1,1)$ \\
controle & dificultadora & preferencial & treinada & $27,8(1,5)$ & $30,2(1,5)$ \\
controle & dificultadora & preferencial & controle & $14,6(1,1)$ & $19,8(1,0)$ \\
controle & dificultadora & não & treinada & $30,9(1,5)$ & $33,6(1,5)$ \\
controle & dificultadora & não & controle & $18,2(1,4)$ & $24,2(1,3)$ \\
\hline inicial & final & preferencial & treinada & $19,0(1,2)$ & $21,4(1,2)$ \\
inicial & final & ambas & controle & $13,2(1,2)$ & $19,1(1,1)$ \\
inicial & final & não & treinada & $28,8(4,1)$ & $32,4(4,5)$ \\
inicial & dificultadora & preferencial & treinada & $15,1(1,3)$ & $19,1(1,3)$ \\
inicial & dificultadora & preferencial & controle & $10,1(1,3)$ & $19,8(1,0)$ \\
inicial & dificultadora & não & treinada & $16,8(1,3)$ & $21,3(1,3)$ \\
inicial & dificultadora & não & controle & $7,8(1,1)$ & $15,3(1,3)$ \\
\hline avançado & final & ambas & treinada & $12,7(1,2)$ & $15,3(1,3)$ \\
avançado & final & ambas & controle & $7,0(0,9)$ & $12,2(1,0)$ \\
avançado & dificultadora & preferencial & treinada & $11,2(1,2)$ & $16,3(1,2)$ \\
avançado & dificultadora & preferencial & controle & $4,8(0,8)$ & $12,5(1,0)$ \\
avançado & dificultadora & não & treinada & $12,6(1,3)$ & $18,2(1,4)$ \\
avançado & dificultadora & não & controle & $5,8(1,1)$ & $15,3(1,4)$ \\
\hline
\end{tabular}

\subsection{Propostas}

O modelo Beta-Binomial/Poisson p-variado considerado em Lora e Singer (2008) apresenta as seguintes restrições: i) a covariância entre duas contagens de totais é constante (quaisquer que sejam tais contagens); ii) não permite a existência de sobredipersão para as contagens de totais; iii) não permite relacionar o total de tentativas com a probabilidade de sucesso, como no modelo descrito em Zhu et al. (2003).

Tais restrições motivam generalizações. A primeira poderia ser concretizada por um modelo que permitisse covariâncias diferentes entre as contagens de totais nos difer- 
entes instantes de observação, bem como sobredispersão para as contagens de totais. No exemplo, essas características são sugeridas pelos dados. Outra direção para generalização seria um modelo que considerasse alguma forma de relação entre o total de tentativas e a probabilidade de sucesso, já que o treinamento descrito para os participantes pode fazer com que um ganho na agilidade esteja relacionado com uma maior probabilidade de acerto das sequências.

Para possibilitar covariâncias diferentes e sobredispersão para os totais, no capítulo 2 propomos um modelo Beta-Binomial/Poisson-Gama. No capítulo 3 generalizamos o modelo Beta-Binomial/Poisson-Gama incorporando um parâmetro para relacionar tanto o total de tentativas com a probabilidade esperada de sucesso, quanto com a probabilidade individual de sucesso. Tais modelos representam a extensão longitudinal da proposta de Zhu et al. (2003). 


\section{Capítulo 2}

\section{Modelo}

\section{Beta-Binomial/Poisson-Gama}

Apresentamos o modelo Beta-Binomial/Poisson-Gama, construído para permitir que a covariância entre os totais de tentativas sejam diferentes nas diversas situações de contagens, e também para considerar a sobredispersão para o total de tentativas; situações estas que não são previstas pelo modelo Beta-Binomial/Poisson p-variado.

\subsection{Modelo}

Considerando o vetor (1.1) para o $g$-ésimo indivíduo, assumimos agora, adicionalmente às distribuições (1.2) e (1.3), que para todo $g$ e $h$,

$$
\begin{aligned}
& N_{g h} \mid \tau_{g} \sim \operatorname{Poisson}\left(\lambda\left(\mathbf{z}_{\lambda g h}\right) \tau_{g}\right) \text { independentes } \\
& \tau_{g} \sim \operatorname{Gama}\left(\alpha\left(\mathbf{z}_{\alpha g}\right) / \delta\left(\mathbf{z}_{\delta g}\right), 1 / \delta\left(\mathbf{z}_{\delta g}\right)\right) \text { independentes }
\end{aligned}
$$

em que $\mathbf{z}_{\lambda g h}, \mathbf{z}_{\alpha g}$ e $\mathbf{z}_{\delta g}$ são vetores de covariáveis.

No modelo Beta-Binomial/Poisson p-variado, as distribuições em (1.2) e (1.3) permitem que as probabilidades de sucesso sejam diferentes para indivíduos do mesmo grupo, porém geradas por uma mesma distribuição. A modelagem proposta em (2.1) e (2.2), baseada em Nelson (1985), permite também que os totais de tentativas sejam diferentes, mas gerados por uma mesma distribuição para indivíduos do mesmo grupo. 
Assim como na parametrização da distribuição Beta, a parametrização em (2.2) para a distribuição Gama foi adotada para facilitar a estimação via máxima verossimilhança. A relação entre os parâmetros $\alpha$ e $\delta$ e os parâmetros usualmente utilizados para a distribuição Gama $(a, b)$, como em Mood et al (1974), por exemplo, é dada por

$$
\alpha=\frac{a}{b} \quad(\alpha>0) \quad \text { e } \quad \delta=\frac{1}{b} \quad(\delta>0) .
$$

Os momentos centrais de primeira e segunda ordens de (2.2) são

$$
\begin{aligned}
& E\left(\tau_{g}\right)=\alpha\left(\mathbf{z}_{\alpha g}\right) \\
& \operatorname{Var}\left(\tau_{g}\right)=\alpha\left(\mathbf{z}_{\alpha g}\right) \delta\left(\mathbf{z}_{\delta g}\right)
\end{aligned}
$$

De (2.1) e (2.2), podemos concluir que os momentos centrais de primeira e segunda ordens para o total de tentativas são

$$
\begin{aligned}
& E\left(N_{g h}\right)=\lambda\left(\mathbf{z}_{\lambda g h}\right) \alpha\left(\mathbf{z}_{\alpha g}\right) \\
& \operatorname{Var}\left(N_{g h}\right)=\lambda\left(\mathbf{z}_{\lambda g h}\right) \alpha\left(\mathbf{z}_{\alpha g}\right)\left\{1+\lambda\left(\mathbf{z}_{\lambda g h}\right) \delta\left(\mathbf{z}_{\delta g}\right)\right\} \\
& \operatorname{Cov}\left(N_{g h}, N_{g h^{\prime}}\right)=\lambda\left(\mathbf{z}_{\lambda g h}\right) \lambda\left(\mathbf{z}_{\lambda g h^{\prime}}\right) \alpha\left(\mathbf{z}_{\alpha g}\right) \delta\left(\mathbf{z}_{\delta g}\right)
\end{aligned}
$$

para todo $g, h, h^{\prime}, h \neq h^{\prime}$

Da mesma maneira que na seção 1.1, de (1.2) e (1.3), obtemos

$$
X_{g h} \mid N_{g h} \sim \text { Beta - Binomial }\left\{N_{g h}, \mu\left(\mathbf{z}_{\mu g h}\right), \theta\left(\mathbf{z}_{\theta g h}\right)\right\}
$$

e portanto, os momentos centrais de primeira e segunda ordens para as contagens de acertos, para todo $g$ e $h$, são

$$
\begin{aligned}
E\left(X_{g h}\right)= & \mu\left(\mathbf{z}_{\mu g h}\right) \lambda\left(\mathbf{z}_{\lambda g h}\right) \alpha\left(\mathbf{z}_{\alpha g}\right) \\
\operatorname{Var}\left(X_{g h}\right)= & \frac{\mu\left(\mathbf{z}_{\mu g h}\right) \lambda\left(\mathbf{z}_{\lambda g h}\right) \alpha\left(\mathbf{z}_{\alpha g}\right)}{1+\theta\left(\mathbf{z}_{\theta g h}\right)}\left\{1+\theta\left(\mathbf{z}_{\theta g h}\right)\left[1+\lambda\left(\mathbf{z}_{\lambda g h}\right) \alpha\left(\mathbf{z}_{\alpha g}\right)\right.\right. \\
& \left.\left.+\lambda\left(\mathbf{z}_{\lambda g h}\right) \alpha\left(\mathbf{z}_{\alpha g}\right) \mu\left(\mathbf{z}_{\mu g h}\right)\right]+\lambda\left(\mathbf{z}_{\lambda g h}\right) \delta\left(\mathbf{z}_{\delta g}\right)\left[\theta\left(\mathbf{z}_{\theta g h}\right)+\mu\left(\mathbf{z}_{\mu g h}\right)\right]\right\} \\
\operatorname{Cov}\left(X_{g h}, X_{g h^{\prime}}\right)= & \mu\left(\mathbf{z}_{\mu g h}\right) \mu\left(\mathbf{z}_{\mu g h^{\prime}}\right) \lambda\left(\mathbf{z}_{\lambda g h}\right) \lambda\left(\mathbf{z}_{\lambda g h^{\prime}}\right) \alpha\left(\mathbf{z}_{\alpha g}\right) \delta\left(\mathbf{z}_{\delta g}\right)
\end{aligned}
$$

para todo $g, h, h^{\prime}, h \neq h^{\prime}$.

De (2.8) observamos que o valor esperado do número de acertos é função de três componentes: o primeiro $\left[\mu\left(\mathbf{z}_{\mu g h}\right)\right]$ ligado à capacidade de acerto e os dois últimos $\left[\lambda\left(\mathbf{z}_{\lambda g h}\right)\right.$ e $\alpha\left(\mathbf{z}_{\alpha g}\right)$ ], à agilidade. 
O parâmetro $\theta\left(\mathbf{z}_{\theta g h}\right)$, além de estar relacionado com a variabilidade da probabilidade de sucesso, também está relacionado com a sobredispersão do número de sucessos. Essa sobredispersão também depende do parâmetro $\delta\left(\mathbf{z}_{\delta g}\right)$. Quando tanto $\theta\left(\mathbf{z}_{\theta g h}\right)$ quanto $\delta\left(\mathbf{z}_{\delta g}\right)$ são iguais a zero, não há sobredispersão para tal contagem.

O parâmetro $\delta\left(\mathbf{z}_{\delta g}\right)$, por outro lado, também está relacionado com a variabilidade e com a sobredispersão do total de tentativas, de maneira que, quando $\delta\left(\mathbf{z}_{\delta g}\right)=0$, não há sobredispersão para tal contagem. Além disso, o parâmetro $\delta\left(\mathbf{z}_{\delta g}\right)$ governa tanto a covariância entre as contagens dos totais de tentativas, quanto aquela entre as contagens de acertos. Quando $\delta\left(\mathbf{z}_{\delta g}\right)=0$, essas contagens são independentes.

A covariância entre o número de sucessos e o total de tentativas é

$$
\operatorname{Cov}\left(X_{g h}, N_{g h}\right)=\mu\left(\mathbf{z}_{\mu g h}\right) \lambda\left(\mathbf{z}_{\lambda g h}\right) \alpha\left(\mathbf{z}_{\alpha g}\right)\left\{1+\lambda\left(\mathbf{z}_{\lambda g h}\right) \delta\left(\mathbf{z}_{\delta g}\right)\right\}
$$

Para investigar os efeitos das covariáveis, adotamos os modelos log-lineares (1.15), (1.16) e

$$
\begin{aligned}
& \lambda\left(\mathbf{z}_{\lambda g h}\right)=\exp \left(\mathbf{z}_{\lambda g h}^{\prime} \boldsymbol{\beta}_{\lambda}\right) \\
& \alpha\left(\mathbf{z}_{\alpha g}\right)=\exp \left(\mathbf{z}_{\alpha g}^{\prime} \boldsymbol{\beta}_{\alpha}\right) \\
& \delta\left(\mathbf{z}_{\delta g}\right)=\exp \left(\mathbf{z}_{\delta g}^{\prime} \boldsymbol{\beta}_{\delta}\right)
\end{aligned}
$$

em que $\boldsymbol{\beta}_{\lambda}, \boldsymbol{\beta}_{\alpha}$ e $\boldsymbol{\beta}_{\delta}$ são vetores de parâmetros a serem estimados.

De (1.2), (1.3), (2.1) e (2.2), segue que a distribuição conjunta do número de sucessos e tentativas para o $g$-ésimo participante é

$$
\begin{gathered}
P\left(X_{g 1}, N_{g 1}, \ldots, X_{g p}, N_{g p}\right)=\prod_{h=1}^{p} P\left(X_{g h} \mid N_{g h}\right) P\left(N_{g 1}, \ldots, N_{g p}\right)= \\
\quad=\prod_{h=1}^{p} P\left(X_{g h} \mid N_{g h}\right)\left(\int_{0}^{\infty} \prod_{h=1}^{p} P\left(N_{g h} \mid \tau_{h}\right) f\left(\tau_{g}\right) d \tau_{g}\right)
\end{gathered}
$$

com $f$ denotando a densidade de (2.2). Como o logaritmo da verossimilhança é dado por

$$
\begin{aligned}
& \log L\left(\boldsymbol{\beta}_{\mu}, \boldsymbol{\beta}_{\theta}, \boldsymbol{\beta}_{\lambda}, \boldsymbol{\beta}_{\alpha}, \boldsymbol{\beta}_{\delta}\right)= \\
= & \sum_{g=1}^{m} \sum_{h=1}^{p} \log P\left(X_{g h} \mid N_{g h}, \boldsymbol{\beta}_{\mu}, \boldsymbol{\beta}_{\theta}\right)+\sum_{g=1}^{m} \log P\left(N_{g 1}, \ldots, N_{g p} \mid \boldsymbol{\beta}_{\lambda}, \boldsymbol{\beta}_{\alpha}, \boldsymbol{\beta}_{\delta}\right),
\end{aligned}
$$


neste caso também, os parâmetros da distribuição Beta-Binomial podem ser estimados separadamente dos parâmetros da distribuição Poisson-Gama. No primeiro caso, a estimação dos parâmetros segue as mesmas linhas apresentadas em Lora e Singer (2008). A distribuição dos totais de tentativas sob o modelo Poisson/Gama, considerando (2.1) e (2.2), pode ser escrita como

$$
\begin{aligned}
& P\left(N_{g 1}=n_{g 1}, \ldots, N_{g p}=n_{g p} \mid \boldsymbol{\beta}_{\lambda}, \boldsymbol{\beta}_{\alpha}, \boldsymbol{\beta}_{\delta}\right)= \\
& =\prod_{h=1}^{p}\left\{\frac{\left[\lambda\left(\mathbf{z}_{\lambda g h}\right)\right]^{n_{g h}}}{n_{g h} !}\right\}\left[\frac{1}{\delta\left(\mathbf{z}_{\delta g}\right)}\right]^{\alpha\left(\mathbf{z}_{\alpha g}\right) / \delta\left(\mathbf{z}_{\delta g}\right)} \Gamma\left(\sum_{h=1}^{p} n_{g h}+\frac{\alpha\left(\mathbf{z}_{\alpha g}\right)}{\delta\left(\mathbf{z}_{\delta g}\right)}\right)\left\{\Gamma\left(\frac{\alpha\left(\mathbf{z}_{\alpha g}\right)}{\delta\left(\mathbf{z}_{\delta g}\right)}\right)\right\}^{-1} \\
& \div\left[\sum_{h=1}^{p} \lambda\left(\mathbf{z}_{\lambda g h}\right)+\frac{1}{\delta\left(\mathbf{z}_{\delta g}\right)}\right]^{\Sigma_{h=1}^{p} n_{g h}+\alpha\left(\mathbf{z}_{\alpha g}\right) / \delta\left(\mathbf{z}_{\delta g}\right)} \\
& =\prod_{h=1}^{p}\left\{\frac{\left[\lambda\left(\mathbf{z}_{\lambda g h}\right)\right]^{n_{g h}}}{n_{g h} !}\right\} \prod_{u=0}^{\Sigma_{h=1}^{p} n_{g h}-1}\left[\alpha\left(\mathbf{z}_{\alpha g}\right)+u \delta\left(\mathbf{z}_{\delta g}\right)\right] \\
& \div\left\{\delta\left(\mathbf{z}_{\delta g}\right)\left[\sum_{h=1}^{p} \lambda\left(\mathbf{z}_{\lambda g h}\right)\right]+1\right.
\end{aligned}
$$

Em (2.15), a simplificação para a razão entre as duas funções gama faz sentido quando $\sum_{h=1}^{p} n_{g h} \neq 0$. Caso esta condição não esteja satisfeita, à razão entre as funções gama será atribuído o valor 1 , não alterando o valor da probabilidade de $\left(N_{g 1}, \ldots, N_{g p}\right)^{\prime}$.

O núcleo da função log-verossimilhança é

$$
\begin{aligned}
& L\left(\beta_{\lambda}, \beta_{\alpha}, \beta_{\delta}\right)=\sum_{g=1}^{m} \log P\left(N_{g 1}, \ldots, N_{g h}\right)= \\
& =\sum_{g=1}^{m}\left\{\sum_{h=1}^{p}\left[n_{g h} \log \lambda\left(\mathbf{z}_{\lambda g h}\right)\right]+\sum_{u=0}^{\Sigma_{h=1}^{p} n_{g h}-1} \log \left[\alpha\left(\mathbf{z}_{\alpha g}\right)+u \delta\left(\mathbf{z}_{\delta g}\right)\right]\right. \\
& \left.-\left[\sum_{h=1}^{p} n_{g h}+\frac{\alpha\left(\mathbf{z}_{\alpha g}\right)}{\delta\left(\mathbf{z}_{\delta g}\right)}\right] \log \left[\delta\left(\mathbf{z}_{\delta g}\right)\left(\sum_{h=1}^{p} \lambda\left(\mathbf{z}_{\lambda g h}\right)\right)+1\right]\right\}
\end{aligned}
$$

Para a maximização de (2.16) e obtenção dos estimadores de $\boldsymbol{\beta}_{\lambda}, \boldsymbol{\beta}_{\alpha}$ e $\boldsymbol{\beta}_{\delta}$, adotamos um processo iterativo de Newton-Raphson. As primeiras e segundas derivadas de (2.16) usadas para esse processo estão apresentadas no Apêndice B. Estimativas obtidas pelo método dos momentos podem ser usadas como valores iniciais para $\lambda\left(\mathbf{z}_{\lambda h k l}\right), \alpha\left(\mathbf{z}_{\alpha h}\right)$ e $\delta\left(\mathbf{z}_{\delta h}\right)$. Testes da razão de verossimilhanças podem ser usados para redução do modelo. 
O processo iterativo foi implementado no software $\mathrm{R}$ e o código correspondente pode ser obtido em http://www.ime.usp.br/ jmsinger.

\subsection{Análise do exemplo por meio do modelo Beta- Binomial/Poisson-Gama}

Para analisar o exemplo, ajustamos modelos log-lineares da forma de 1.15, 1.16, 1.17 e usamos as mesmas estratégias de eliminação dos termos não significantes. A indexação dos parâmetros e a cela de referência usadas são as mesmas apresentadas na seção 1.1.

A modelagem do número de acertos corresponde àquela apresentada na seção 1.1; as estimativas para a probabilidade esperada de sucesso, $\mu\left(\mathbf{z}_{\mu g h}\right)$, e para os valores esperados do parâmetro $\theta\left(\mathbf{z}_{\theta g h}\right)$ sob o modelo final podem ser obtidas, respectivamente, das Tabelas 1.4 e 1.5 .

Para o total de tentativas, o vetor de parâmetros inicial é $\boldsymbol{\beta}=\left(\boldsymbol{\beta}_{\lambda}, \boldsymbol{\beta}_{\alpha}, \boldsymbol{\beta}_{\delta}\right)$ em que

$$
\begin{aligned}
\boldsymbol{\beta}_{\lambda}= & \left(\beta_{\lambda 0}, \beta_{\lambda 1}, \beta_{\lambda 2}, \beta_{\lambda N}, \beta_{\lambda D}, \beta_{\lambda C},\right. \\
& \left.\beta_{\lambda(1 * D)}, \beta_{\lambda(1 * N)}, \beta_{\lambda(1 * C)}, \beta_{\lambda(2 * D)}, \beta_{\lambda(2 * N)}, \beta_{\lambda(2 * C)}, \beta_{\lambda(D * N)}, \beta_{\lambda(D * C)}, \beta_{\lambda(N * C)}\right) \\
\boldsymbol{\beta}_{m}= & \left(\beta_{m 0}, \beta_{m 1}, \beta_{m 2}, \beta_{m N}, \beta_{m(1 * N)}, \beta_{m(2 * N)}\right)
\end{aligned}
$$

com $m=\alpha, \delta$. Para o vetor de parâmetros relacionados a $\lambda$, por exemplo, $\beta_{\lambda 0}$ corresponde ao logaritmo de $\lambda$ para indivíduos controle, usando a mão preferencial, realizando a sequência controle durante a avaliação final; $\beta_{\lambda N}$ corresponde ao acréscimo no logaritmo de $\lambda$ devido ao efeito do uso da mão não preferencial em relação ao uso da preferencial; e $\beta_{\lambda(1 * N)}$ corresponde à interação entre o estágio inicial da doença (1) e o uso da mão não preferencial $(N)$. Os elementos relacionados aos diferentes instantes de contagens (representados pelos índices $D$ e $C$ ) do vetor $\boldsymbol{\beta}_{\lambda}$ permitem que os totais de tentativas esperados sejam diferentes nesses diferentes instantes de avaliação. Por outro lado, $\alpha\left(\mathbf{z}_{\alpha g}\right)$ e $\delta\left(\mathbf{z}_{\delta g}\right)$ não variam nos diferentes instantes de avaliação; os vetores $\boldsymbol{\beta}_{\alpha}$ e $\boldsymbol{\beta}_{\delta}$ não apresentam elementos que diferenciem tais instantes, apresentando apenas aqueles que permitem a comparação dos grupos de participantes.

Como no caso do modelo Beta-Binomial/Poisson p-variado, o processo iterativo 
proposto mostrou-se sensível aos valores iniciais, especialmente para as interações. A mesma estratégia descrita da seção 1.1 foi usada para o ajuste do modelo inicial.

As interações de primeira ordem não significativas foram identificadas e sua eliminação pode ser justificada pelo resultado $(p=0,765)$ do teste da hipótese

$$
\begin{aligned}
H_{0}: & \beta_{\lambda(1 * D)}, \beta_{\lambda(1 * N)}, \beta_{\lambda(1 * C)}, \beta_{\lambda(2 * D)}, \beta_{\lambda(2 * N)}, \beta_{\lambda(2 * C)}, \beta_{\lambda(D * N)}, \beta_{\lambda(D * C)}, \beta_{\lambda(N * C)}, \\
& \beta_{\alpha(1 * N)}, \beta_{\alpha(2 * N)}, \beta_{\delta(2 * N)}=0
\end{aligned}
$$

Sob o modelo reduzido, os efeitos principais não significativos foram identificados e sua eliminação pode ser justificada pelo resultado $(p=0,506)$ do teste da hipótese

$$
H_{0}: \beta_{\alpha 1}, \beta_{\alpha 2}, \beta_{\alpha N}, \beta_{\delta 1}, \beta_{\delta 2}=0
$$

A qualidade do ajuste do modelo final pode ser justificada por um teste da razão de verossimilhanças comparando-o com o inicial $(p=0,765)$. As estimativas do modelo final com respectivos erros padrões estão apresentadas na Tabela 2.1.

Tabela 2.1: Estimativas dos parâmetros sob o modelo Poisson-Gama final e erros padrões

\begin{tabular}{llcc}
\hline Parâmetro & Relacionado ao & Estimativa & Erro Padrão \\
\hline$\beta_{\lambda 0}$ & grupo controle, mão preferencial, & 2,42 & 0,04 \\
& avaliação final e sequência treinada & & \\
$\beta_{\lambda 1}$ & efeito do estágio inicial & $-0,39$ & 0,04 \\
$\beta_{\lambda 2}$ & efeito do estágio avançado & $-0,64$ & 0,05 \\
$\beta_{\lambda D}$ & efeito da condição dificultadora & $-0,08$ & 0,04 \\
$\beta_{\lambda N}$ & efeito da mão não preferencial & 0,10 & 0,04 \\
$\beta_{\lambda C}$ & efeito da sequência controle & $-0,25$ & 0,04 \\
\hline$\beta_{\alpha 0}$ & grupo controle, mão preferencial & 1,05 & 0,05 \\
\hline$\beta_{\delta 0}$ & grupo controle, mão preferencial & $-2,01$ & 0,33 \\
$\beta_{\delta 2}$ & efeito do estágio avançado & 1,58 & 0,54 \\
$\beta_{\delta(1 * N)}$ & efeito do estágio inicial e mão não preferencial & 1,46 & 0,64 \\
\hline
\end{tabular}

A partir das estimativas da Tabela 2.1 pode-se calcular os valores esperados de $\lambda\left(\mathbf{z}_{\lambda g h}\right)$ (Tabela 2.2) e os de $\delta\left(\mathbf{z}_{\delta g}\right)$ (Tabela 2.3). Além disso, como apenas o parâmetro $\beta_{\alpha 0}$ foi incluído no modelo final, temos $\alpha\left(\mathbf{z}_{\alpha g}\right)=2,9$, com erro padrão de 0,1 , qualquer que seja o estágio da doença ou mão usada. 
Tabela 2.2: Estimativas dos valores esperados de $\lambda\left(\mathbf{z}_{\lambda g h}\right)$

\begin{tabular}{|c|c|c|c|c|c|}
\hline $\begin{array}{c}\text { Estágio } \\
\text { da doença }\end{array}$ & Avaliação & Mão & Sequência & $\begin{array}{c}\text { Valor } \\
\text { esperado }\end{array}$ & $\begin{array}{c}\text { Erro } \\
\text { padrão }\end{array}$ \\
\hline controle & final & preferencial & treinada & 11,2 & 0,5 \\
\hline controle & final & preferencial & controle & 8,7 & 0,4 \\
\hline controle & final & não & treinada & 12,4 & 0,5 \\
\hline controle & final & não & controle & 9,6 & 0,4 \\
\hline controle & dificultadora & preferencial & treinada & 10,3 & 0,4 \\
\hline controle & dificultadora & preferencial & controle & 8,0 & 0,3 \\
\hline controle & dificultadora & não & treinada & 11,4 & 0,4 \\
\hline controle & dificultadora & não & controle & 8,9 & 0,3 \\
\hline inicial & final & preferencial & treinada & 7,6 & 0,4 \\
\hline inicial & final & preferencial & controle & 5,9 & 0,3 \\
\hline inicial & final & não & treinada & 8,4 & 0,4 \\
\hline inicial & final & não & controle & 6,5 & 0,3 \\
\hline inicial & dificultadora & preferencial & treinada & 7,0 & 0,3 \\
\hline inicial & dificultadora & preferencial & controle & 5,4 & 0,3 \\
\hline inicial & dificultadora & não & treinada & 7,7 & 0,4 \\
\hline inicial & dificultadora & não & controle & 6,0 & 0,3 \\
\hline avançado & final & preferencial & treinada & 5,9 & 0,3 \\
\hline avançado & final & preferencial & controle & 4,6 & 0,2 \\
\hline avançado & final & não & treinada & 6,5 & 0,4 \\
\hline avançado & final & não & controle & 5,1 & 0,3 \\
\hline avançado & dificultadora & preferencial & treinada & 5,5 & 0,3 \\
\hline avançado & dificultadora & preferencial & controle & 4,2 & 0,2 \\
\hline avançado & dificultadora & não & treinada & 6,0 & 0,3 \\
\hline avançado & dificultadora & não & controle & 4,7 & 0,3 \\
\hline
\end{tabular}


Tabela 2.3: Estimativas dos valores esperados de $\delta\left(\mathbf{z}_{\delta g}\right)$

\begin{tabular}{cccc}
\hline $\begin{array}{c}\text { Estágio } \\
\text { da doença }\end{array}$ & Mão & $\begin{array}{c}\text { Valor } \\
\text { esperado }\end{array}$ & $\begin{array}{c}\text { Erro } \\
\text { padrão }\end{array}$ \\
\hline controle & ambas & 0,13 & 0,04 \\
\hline inicial & preferencial & 0,13 & 0,04 \\
inicial & não & 0,58 & 0,32 \\
\hline avançado & ambas & 0,65 & 0,28 \\
\hline
\end{tabular}

Comparando os resultados obtidos para o total de tentativas esperado sob o modelo Beta-Binomial/Poisson-Gama com aqueles obtidos sob o modelo Beta-Binomial/Poisson p-variado, podemos inicialmente notar que com primeiro detectamos redução nesse total para os participantes em estágio inicial da doença e uma redução ainda maior para aqueles em estágio avançado, relativamente ao grupo controle $\left(\beta_{\lambda 2}<\beta_{\lambda 1}<0\right.$ e $\beta_{\alpha 1}=\beta_{\alpha 2}=$ 0). Sob o modelo Beta-Binomial/Poisson p-variado não detectamos tal diferença para participantes em estágio inicial da doença e controles, quando a sequência controle era realizada sob a condição dificultadora. Além disso, com os dois modelos detectamos redução no total de tentativas esperado para a seqüência controle, quando comparada com a treinada $\left(\beta_{\lambda C}<0\right)$; e sob a condição dificultadora, quando comparada com a avaliação final $\left(\beta_{\lambda D}<0\right)$. Por outro lado, sob o modelo Beta-Binomial/Poisson-Gama pudemos detectar que os participantes que usam a mão não preferencial têm maior valor esperado do total de tentativas do que os usando a mão preferencial $\left(\beta_{\lambda N}>0\right.$ e $\left.\beta_{\alpha N}=0\right)$; sob o modelo Beta-Binomial/Poisson p-variado não detectamos tal aumento apenas sob a condição dificultadora realizando a sequência controle, e na avaliação final realizando a sequência treinada só detectamos tal aumento para os indivíduos em estágio inicial da doença.

Comparando as estimativas para os valores esperados de acertos e totais de tentativas sob os dois modelos podemos dizer que na maioria dos casos ambos geraram estimativas semelhantes. A maior diferença foi observada para os indivíduos em estágio inicial da doença, na avaliação final, realizando a sequência treinada com a mão não preferencial, situação em que o modelo Beta-Binomial/Poisson $p$-variado superestima tanto o número de acertos, quanto o total de tentativas (cerca de $50 \%$ maior do que as médias 
observadas). Os erros padrões obtidos também foram semelhantes sob os dois modelos.

Para o parâmetro relativo à dispersão, $\delta\left(\mathbf{z}_{\delta g}\right)$, presente apenas no modelo BetaBinomail/Poisson-Gama, não há diferença entre participantes do grupo controle e em estágio inicial da doença $\left(\beta_{\delta 1}=0\right)$, nem entre os usando ou não a mão preferencial $\left(\beta_{\delta N}=0\right)$. Os participantes em estágio avançado da doença têm dispersão esperada maior que os outros $\left(\beta_{\delta 2}>0\right)$, assim como aqueles do estágio inicial usando a mão não preferencial $\left(\beta_{\delta(1 * N)}>0\right)$.

Além das generalizações permitidas pelo modelo Poisson-Gama (covariâncias diferentes entre os totais de tentativas e sobredispersão para esses totais), este modelo, se comparado ao Poisson p-variado, simplifica a comparação do total de tentativas nos diferentes instantes de avaliação. Enquanto o modelo Poisson $p$-variado é construído com um vetor de parâmetros para cada instante de avaliação, o modelo Poisson-Gama engloba todos os instantes usando um único vetor. Assim, para comparar o total de tentativas usando o modelo Poisson $p$-variado é necessário reescrevê-lo, tornando-o específico para problemas com estruturas similares. Já com o modelo Poisson-Gama, a comparação dos totais de tentativas esperadas nos diferentes instantes de observação é feita simplesmente com a eliminação de parâmetros do modelo de regressão relacionados, tornando-o portanto mais simples e mais flexível do que o primeiro. 
Tabela 2.4: Estimativas dos valores esperados do número de acertos e total de tentativas, e respectivos erros padrões (entre parênteses)

\begin{tabular}{cccccc}
\hline $\begin{array}{c}\text { Estágio } \\
\text { da doença }\end{array}$ & Avaliação & Mão & Sequência & Acertos & Totais \\
\hline controle & final & preferencial & treinada & $30,8(2,7)$ & $32,2(1,7)$ \\
controle & final & preferencial & controle & $21,7(1,7)$ & $25,1(1,4)$ \\
controle & final & não & treinada & $34,1(3,3)$ & $35,5(1,9)$ \\
controle & final & não & controle & $24,0(2,0)$ & $27,7(1,5)$ \\
controle & dificultadora & preferencial & treinada & $27,2(2,4)$ & $29,7(1,6)$ \\
controle & dificultadora & preferencial & controle & $17,4(1,7)$ & $23,1(1,3)$ \\
controle & dificultadora & não & treinada & $30,0(2,9)$ & $32,8(1,7)$ \\
controle & dificultadora & não & controle & $19,2(2,1)$ & $25,5(1,4)$ \\
\hline inicial & final & preferencial & treinada & $19,7(1,7)$ & $21,8(1,3)$ \\
inicial & final & preferencial & controle & $11,7(1,3)$ & $16,9(1,0)$ \\
inicial & final & não & treinada & $21,4(2,1)$ & $24,0(1,5)$ \\
inicial & final & não & controle & $12,9(1,6)$ & $18,7(1,2)$ \\
inicial & dificultadora & preferencial & treinada & $15,8(1,7)$ & $20,1(1,2)$ \\
inicial & dificultadora & preferencial & controle & $8,0(1,2)$ & $15,6(1,0)$ \\
inicial & dificultadora & não & treinada & $17,5(2,1)$ & $22,2(1,4)$ \\
inicial & dificultadora & não & controle & $8,8(1,5)$ & $17,3(1,1)$ \\
\hline avançado & final & preferencial & treinada & $14,1(1,2)$ & $17,0(1,1)$ \\
avançado & final & preferencial & controle & $7,6(0,9)$ & $13,2(0,9)$ \\
avançado & final & não & treinada & $15,5(1,6)$ & $18,7(1,3)$ \\
avançado & final & não & controle & $8,3(1,1)$ & $14,6(1,0)$ \\
avançado & dificultadora & preferencial & treinada & $10,8(1,2)$ & $15,7(1,0)$ \\
avançado & dificultadora & preferencial & controle & $4,7(0,6)$ & $12,2(0,8)$ \\
avançado & dificultadora & não & treinada & $11,9(1,4)$ & $17,3(1,2)$ \\
avançado & dificultadora & não & controle & $5,1(0,7)$ & $13,5(1,0)$ \\
\hline
\end{tabular}




\section{Capítulo 3}

\section{Modelos}

\section{Beta-Binomial/Poisson-Gama com enlace}

Motivados pelo modelo proposto por Zhu et al. (2003), propomos a seguir generalizações dos casos apresentados nos capítulos 1 e 2 que permitem modelar a relação entre a probabilidade de acerto e o total de tentativas.

O primeiro modelo, apresentado na seção 3.1, relaciona o total de tentativas com o valor esperado da probabilidade de sucesso. Na seção 3.2, apresentamos um modelo que relaciona o total de tentativas com a probabilidade individual de sucesso. Comparando os dois modelos, a principal vantagem do primeiro é a estimação de seus parâmetros, que não exige nenhum método numérico de integração, como acontece com o segundo. Por outro lado, este modelo permite que o parâmetro que relaciona o total de tentativas com a probabilidade de sucesso seja diferente para os diversos instantes de avaliação das contagens e para os diversos grupos; o primeiro modelo permite apenas a comparação de tal parâmetro entre os diversos grupos. 


\subsection{Modelo Beta-Binomial/Poisson-Gama com enlace gerado pelas probabilidades esperadas de sucesso}

Considerando o vetor de respostas (1.1), para o $g$-ésimo participante assumimos as distribuições (1.2), (1.3), (2.1), (2.2) e acrescentamos a relação

$$
\alpha_{g}=\exp \left\{\eta_{1}\left(\mathbf{z}_{\eta_{1} g}\right) \log \left(\frac{\bar{\mu}_{g}}{1-\bar{\mu}_{g}}\right)\right\}
$$

em que $\bar{\mu}_{g}$ é a média dos $p$ valores de $\mu\left(\mathbf{z}_{\mu g h}\right)$ para o $g$-ésimo indivíduo e $\mathbf{z}_{\eta_{1} g}$ é um vetor de covariáveis.

Os momentos centrais de primeira e segunda ordem do número de acertos e do total de tentativas, bem como as covariâncias entre essas contagens, se escritas em função de $\alpha_{g}$, são iguais aos apresentados no capítulo 2. As interpretações dos parâmetros $\theta\left(\mathbf{z}_{\theta g}\right)$ e $\delta\left(\mathbf{z}_{\delta g}\right)$ também são as mesmas do modelo apresentado no capítulo 2. Porém, como o valor de $\alpha_{g}$ depende do valor de $\bar{\mu}_{g}$, a estimação dos parâmetros do modelo Poisson-Gama não pode mais ser feita separadamente da estimação dos parâmetros do modelo Beta-Binomial.

O parâmetro $\eta_{1}\left(\mathbf{z}_{\eta_{1} g}\right)$ em (3.1), modela uma possível relação entre a probabilidade esperada de acertos e um dos termos do valor esperado do total de tentativas $\left(\alpha_{g}\right)$. Quando $\eta_{1}\left(\mathbf{z}_{\eta_{1} g}\right)>0$, quanto maior a probabilidade esperada de sucesso, maior o valor esperado do total de tentativas, ocorrendo o contrário quando $\eta_{1}\left(\mathbf{z}_{\eta_{1} g}\right)<0$. Se $\eta_{1}\left(\mathbf{z}_{\eta_{1} g}\right)=0$, não há relação entre a probabilidade esperada de sucesso e o valor esperado do total de tentativas.

Para investigar os efeitos das covariáveis, adotamos os modelos log-lineares (1.15), (1.16), (2.12), (2.14) e

$$
\eta_{1}\left(\mathbf{z}_{\eta_{1} g}\right)=\mathbf{z}_{\eta_{1} g}^{\prime} \boldsymbol{\beta}_{\eta_{1}}
$$

em que $\boldsymbol{\beta}_{\eta_{1}}$ é um vetor de parâmetros a serem estimados.

De (1.2), (1.3), (2.1), (2.2) e (3.1), temos que o núcleo da função log-verossimilhança pode ser escrito como

$$
\begin{aligned}
& L\left(\boldsymbol{\beta}_{\mu}, \boldsymbol{\beta}_{\theta}, \boldsymbol{\beta}_{\lambda}, \boldsymbol{\beta}_{\eta_{1}}, \boldsymbol{\beta}_{\delta}\right) \propto \sum_{g=1}^{m}\left\{\sum _ { h = 1 } ^ { p } \left[\log \Gamma\left(\frac{1}{\theta\left(\mathbf{z}_{\theta g h}\right)}\right)-\log \Gamma\left(\frac{\mu\left(\mathbf{z}_{\mu g h}\right)}{\theta\left(\mathbf{z}_{\theta g h}\right)}\right)\right.\right. \\
& -\log \Gamma\left(\frac{1-\mu\left(\mathbf{z}_{\mu g h}\right)}{\theta\left(\mathbf{z}_{\theta g h}\right)}\right)+\log \Gamma\left(\frac{\mu\left(\mathbf{z}_{\mu g h}\right)}{\theta\left(\mathbf{z}_{\theta g h}\right)}+x_{g h}\right)+\log \Gamma\left(\frac{1-\mu\left(\mathbf{z}_{\mu g h}\right)}{\theta\left(\mathbf{z}_{\theta g h}\right)}+n_{g h}-x_{g h}\right)
\end{aligned}
$$




$$
\begin{aligned}
& \left.-\log \Gamma\left(\frac{1}{\theta\left(\mathbf{z}_{\theta g h}\right)}+n_{g h}\right)+n_{g h} \log \left(\lambda\left(\mathbf{z}_{\lambda g h}\right)\right)\right]+\log \Gamma\left(\frac{\alpha_{g}}{\delta\left(\mathbf{z}_{\delta g}\right)}+\sum_{h=1}^{p} n_{g h}\right) \\
& -\log \Gamma\left(\frac{\alpha_{g}}{\delta\left(\mathbf{z}_{\delta g}\right)}\right)+\sum_{h=1}^{p} n_{g h} \log \left(\delta\left(\mathbf{z}_{\delta g}\right)\right) \\
& \left.-\left(\sum_{h=1}^{p} n_{g h}+\frac{\alpha_{g}}{\delta\left(\mathbf{z}_{\delta g}\right)}\right) \log \left(\delta\left(\mathbf{z}_{\delta g}\right) \sum_{h=1}^{p} \lambda\left(\mathbf{z}_{\lambda g h}\right)+1\right)\right\}
\end{aligned}
$$

Adotamos novamente um processo iterativo de Newton-Raphson para estimar $\boldsymbol{\beta}_{\mu}$, $\boldsymbol{\beta}_{\theta}, \boldsymbol{\beta}_{\lambda}, \boldsymbol{\beta}_{\eta_{1}}$ e $\boldsymbol{\beta}_{\delta}$. As primeiras e segundas derivadas de (3.3) usadas para esse processo estão apresentadas no Apêndice C. Os resultados obtidos com o modelo BetaBinomial/Poisson-Gama do capítulo 2 podem ser usados como valores iniciais para o processo. Como o vetor de parâmetros $\boldsymbol{\beta}_{\eta_{1}}$ foi adicionado apenas neste modelo, podemos atribuir zero como valor inicial para seus parâmetros; o valor zero pode ser usado para este vetor para manter o processo iterativo estável. Testes da razão de verossimilhanças podem ser usados para redução do modelo. O processo iterativo foi implementado no software $\mathrm{R}$ e o código correspondente pode ser obtido em http://www.ime.usp.br/ jmsinger.

\subsection{Análise do exemplo por meio do modelo Beta- Binomial/Poisson-Gama com enlace gerado pelas probabilidades esperadas de sucesso}

Para analisar o exemplo, ajustamos modelos log-lineares semelhantes aos apresentados nas seções 1.1 e 2.2, e usamos as mesmas estratégias de eliminação dos termos não significantes. A indexação dos parâmetros e a cela de referência usadas também são as mesmas apresentadas nas seções 1.1 e 2.2 .

Sob o modelo inicial, o vetor de parâmetros é $\boldsymbol{\beta}=\left(\boldsymbol{\beta}_{\mu}, \boldsymbol{\beta}_{\theta}, \boldsymbol{\beta}_{\lambda}, \boldsymbol{\beta}_{\eta_{1}}, \boldsymbol{\beta}_{\delta}\right)$ em que

$$
\begin{aligned}
\boldsymbol{\beta}_{m}= & \left(\beta_{m 0}, \beta_{m 1}, \beta_{m 2}, \beta_{m N}, \beta_{m D}, \beta_{m C},\right. \\
& \left.\beta_{m(1 * D)}, \beta_{m(1 * N)}, \beta_{m(1 * C)}, \beta_{m(2 * D)}, \beta_{m(2 * N)}, \beta_{m(2 * C)}, \beta_{m(D * N)}, \beta_{m(D * C)}, \beta_{m(N * C)}\right) \\
\boldsymbol{\beta}_{n}= & \left(\beta_{n 0}, \beta_{n 1}, \beta_{n 2}, \beta_{n N}, \beta_{n(1 * N)}, \beta_{n(2 * N)}\right)
\end{aligned}
$$

$\operatorname{com} m=\mu, \theta, \lambda$ e $n=\eta_{1}, \delta$. 
Assim como para os modelos Beta-Binomial/Poisson p-variado e Beta-Binomial/ Poisson-Gama, o processo iterativo proposto mostrou-se sensível aos valores iniciais, não só para as interações, como também para os efeitos principais. Esta maior sensililidade (envolvendo também os efeitos principais) pode ser atribuída à maior complexidade do processo de ajuste dos parâmetros do modelo, já que, diferente dos modelos em 1.1 e 2.1, todos os parâmetros devem ser estimados simultaneamente. Assim, inicialmente ajustamos um modelo apenas com os termos $\beta_{\mu 0}, \beta_{\theta 0}, \beta_{\lambda 0}, \beta_{\eta_{1} 0}$ e $\beta_{\delta 0}$, em seguida acrescentamos os efeitos principais e por fim, as interações de primeira ordem.

As interações de primeira ordem não significativas foram identificadas e sua eliminação foi justificada pelo resultado $(p=0,990)$ do teste da hipótese

$$
\begin{aligned}
H_{0}: & \beta_{\mu(1 * N)}, \beta_{\mu(1 * C)}, \beta_{\mu(2 * D)}, \beta_{\mu(2 * N)}, \beta_{\mu(2 * C)}, \beta_{\mu(N * C)}, \\
& \beta_{\theta(1 * D)}, \beta_{\theta(1 * C)}, \beta_{\theta(2 * N)}, \beta_{\theta(2 * C)}, \beta_{\theta(D * C)}, \beta_{\mu(N * C)}, \\
& \beta_{\lambda(1 * D)}, \beta_{\lambda(1 * N)}, \beta_{\lambda(1 * C)}, \beta_{\lambda(2 * D)}, \beta_{\lambda(2 * N)}, \beta_{\lambda(2 * C)}, \beta_{\lambda(D * N)}, \beta_{\lambda(D * C)}, \beta_{\lambda(N * C)} \\
& \beta_{\eta_{1}(1 * N)}, \beta_{\eta_{1}(2 * N)}, \beta_{\delta(2 * N)}=0
\end{aligned}
$$

Sob o modelo reduzido, os efeitos principais não significativos foram identificados e sua eliminação foi justificada pelo resultado $(p=0,661)$ do teste da hipótese

$$
H_{0}: \beta_{\mu N}, \beta_{\theta C}, \beta_{\delta 2}=0
$$

A qualidade do ajuste do modelo final foi justificada por um teste da razão de verossimilhanças comparando-o com o inicial $(p=0,992)$. As estimativas do modelo final com respectivos erros padrões estão apresentadas na Tabela 3.1. A partir dessas estimativas, pode-se calcular os valores esperados de $\mu\left(\mathbf{z}_{\mu g h}\right)$ (Tabela 3.2), $\theta\left(\mathbf{z}_{\theta g h}\right)$ (Tabela 3.3), $\lambda\left(\mathbf{z}_{\lambda g h}\right)$ (Tabela 3.4), $\eta_{1}\left(\mathbf{z}_{\eta_{1} g}\right)$ (Tabela 3.5) e $\delta\left(\mathbf{z}_{\delta g}\right)$ (Tabela 3.6).

Comparando os resultados obtidos sob os três modelos, Beta-Binomial/Poisson pvariado, Beta-Binomial/Poisson-Gama e Beta-Binomial/Poisson-Gama com com enlace gerado pelas probabilidades esperadas de sucesso, podemos observar, considerando inicialmente o total de tentativas esperado, que com os três modelos detectamos redução nesse total para os participantes em estágio inicial da doença e uma redução ainda maior para aqueles em estágio avançado, comparando ambos com o grupo controle $\left(\beta_{\lambda 2}+\beta_{\eta_{1} 2}<\right.$ 
Tabela 3.1: Estimativas dos parâmetros sob o modelo final Beta-Binomial/Poisson-Gama com com enlace gerado pelas probebilidades esperadas de sucesso e erros padrões

\begin{tabular}{|c|c|c|c|}
\hline Parâmetro & Relacionado ao & Estimativa & Erro Padrão \\
\hline$\beta_{\mu 0}$ & grupo controle, mão pref., aval. final e seq. treinada & 3,39 & 0,14 \\
\hline$\beta_{\mu 1}$ & efeito do estágio inicial & $-0,49$ & 0,11 \\
\hline$\beta_{\mu 2}$ & efeito do estágio avançado & $-1,51$ & 0,10 \\
\hline$\beta_{\mu D}$ & efeito da condição dificultadora & $-1,53$ & 0,13 \\
\hline$\beta_{\mu C}$ & efeito da sequência controle & $-1,81$ & 0,11 \\
\hline$\beta_{\mu(1 * D)}$ & efeito do estágio inicial e condição dificultadora & $-0,90$ & 0,10 \\
\hline$\beta_{\mu(D * N)}$ & efeito da condição dificultadora e mão não preferencial & 0,48 & 0,05 \\
\hline$\beta_{\mu(D * C)}$ & efeito da condição dificultadora e sequência controle & 0,96 & 0,12 \\
\hline$\beta_{\theta 0}$ & grupo controle, mão pref., aval. final e seq. treinada & $-0,76$ & 0,38 \\
\hline$\beta_{\theta 1}$ & efeito do estágio inicial & $-1,21$ & 0,54 \\
\hline$\beta_{\theta 2}$ & efeito do estágio avançado & 1,08 & 0,50 \\
\hline$\beta_{\theta D}$ & efeito da condição dificultadora & 2,36 & 0,54 \\
\hline$\beta_{\theta N}$ & efeito da mão não preferencial & $-1,79$ & 0,48 \\
\hline$\beta_{\theta(1 * N)}$ & efeito do estágio inicial e mão não preferencial & 2,75 & 0,67 \\
\hline$\beta_{\theta(2 * D)}$ & efeito do estágio avançado e condição dificultadora & $-2,60$ & 0,73 \\
\hline$\beta_{\theta(D * N)}$ & efeito da condição dificultadora e mão não preferencial & $-1,34$ & 0,65 \\
\hline$\beta_{\lambda 0}$ & grupo controle, mão pref., aval. final e seq. treinada & 2,49 & 0,04 \\
\hline$\beta_{\lambda 1}$ & efeito do estágio inicial & 1,49 & 0,05 \\
\hline$\beta_{\lambda 2}$ & efeito do estágio avançado & 0,53 & 0,05 \\
\hline$\beta_{\lambda D}$ & efeito da condição dificultadora & $-0,08$ & 0,03 \\
\hline$\beta_{\lambda N}$ & efeito da mão não preferencial & 0,74 & 0,04 \\
\hline$\beta_{\lambda C}$ & efeito da sequência controle & $-0,25$ & 0,04 \\
\hline$\beta_{\eta_{1} 0}$ & grupo controle, mão preferencial & 0,57 & 0,07 \\
\hline$\beta_{\eta_{1} 1}$ & efeito do estágio inicial & $-1,76$ & 0,13 \\
\hline$\beta_{\eta_{1} 2}$ & efeito do estágio avançado & $-1,35$ & 0,26 \\
\hline$\beta_{\eta_{1} N}$ & efeito da mão não preferencial & $-0,40$ & 0,07 \\
\hline$\beta_{\delta 0}$ & grupo controle, mão preferencial & $-1,53$ & 0,37 \\
\hline$\beta_{\delta 1}$ & efeito do estágio inicial & $-2,52$ & 0,85 \\
\hline$\beta_{\delta N}$ & efeito da mão não preferencial & $-1,87$ & 0,59 \\
\hline$\beta_{\delta(1 * N)}$ & efeito do estágio inicial e mão não preferencial & 2,67 & 1,11 \\
\hline
\end{tabular}

Códigos: pref.: preferencial, aval.: avaliação e seq.: seqüência 
Tabela 3.2: Estimativas dos valores esperados de $\mu\left(\mathbf{z}_{\mu g h}\right)$

\begin{tabular}{cccccc}
\hline $\begin{array}{c}\text { Estágio } \\
\text { da doença }\end{array}$ & Avaliação & Mão & Seqüência & $\begin{array}{c}\text { Valor } \\
\text { esperado }\end{array}$ & $\begin{array}{c}\text { Erro } \\
\text { padrão }\end{array}$ \\
\hline controle & final & ambas & treinada & 0,97 & 0,01 \\
controle & final & ambas & controle & 0,83 & 0,01 \\
controle & dificultadora & preferencial & treinada & 0,87 & 0,01 \\
controle & dificultadora & preferencial & controle & 0,73 & 0,02 \\
controle & dificultadora & não & treinada & 0,91 & 0,01 \\
controle & dificultadora & não & controle & 0,82 & 0,01 \\
\hline inicial & final & ambas & treinada & 0,95 & 0,01 \\
inicial & final & ambas & controle & 0,75 & 0,01 \\
inicial & dificultadora & preferencial & treinada & 0,61 & 0,01 \\
inicial & dificultadora & preferencial & controle & 0,41 & 0,01 \\
inicial & dificultadora & não & treinada & 0,72 & 0,01 \\
inicial & dificultadora & não & controle & 0,52 & 0,01 \\
\hline avançado & final & ambas & treinada & 0,87 & 0,01 \\
avançado & final & ambas & controle & 0,52 & 0,02 \\
avançado & dificultadora & preferencial & treinada & 0,59 & 0,02 \\
avançado & dificultadora & preferencial & controle & 0,38 & 0,02 \\
avançado & dificultadora & não & treinada & 0,70 & 0,01 \\
avançado & dificultadora & não & controle & 0,50 & 0,01 \\
\hline
\end{tabular}


Tabela 3.3: Estimativas dos valores esperados de $\theta\left(\mathbf{z}_{\theta g h}\right)$

\begin{tabular}{cccccc}
\hline $\begin{array}{c}\text { Estágio } \\
\text { da doença }\end{array}$ & Avaliação & Mão & Seqüência & $\begin{array}{c}\text { Valor } \\
\text { esperado }\end{array}$ & $\begin{array}{c}\text { Erro } \\
\text { padrão }\end{array}$ \\
\hline controle & final & preferencial & ambas & 0,47 & 0,18 \\
controle & final & não & ambas & 0,08 & 0,03 \\
controle & dificultadora & preferencial & ambas & 4,98 & 2,47 \\
controle & dificultadora & não & ambas & 0,22 & 0,07 \\
\hline inicial & final & preferencial & ambas & 0,14 & 0,08 \\
inicial & final & não & ambas & 0,36 & 0,16 \\
inicial & dificultadora & preferencial & ambas & 1,48 & 0,58 \\
inicial & dificultadora & não & ambas & 1,01 & 0,38 \\
\hline avançado & final & preferencial & ambas & 1,39 & 0,62 \\
avançado & final & não & ambas & 0,23 & 0,12 \\
avançado & dificultadora & preferencial & ambas & 1,10 & 0,45 \\
avançado & dificultadora & não & ambas & 0,29 & 0,21 \\
\hline
\end{tabular}

$\left.\beta_{\lambda 1}+\beta_{\eta_{1} 1}<0\right)$. Além disso, sob os três modelos detectamos redução no total esperado de tentativas para a seqüência controle, quando comparada com a treinada $\left(\beta_{\lambda C}<0\right)$ e sob a condição dificultadora, quando comparada com a avaliação final $\left(\beta_{\lambda D}<0\right)$. Ainda considerando o total de tentativas, sob os modelos Beta-Binomial/Poisson-Gama e BetaBinomial/Poisson-Gama com enlace gerado pelas probabilidades esperadas de sucesso detectamos o aumento do total de tentativas esperado quando os indivíduos usam a mão não preferencial $\left(\beta_{\lambda N}+\beta_{\eta_{1} N}>0\right)$.

Considerando agora a probabilidade esperada de acerto, como o modelo BetaBinomial/Poisson p-variado é igual ao Beta-Binomial/Poisson-Gama, basta compará-los com o Beta-Binomial/Poisson-Gama com enlace gerado pelas probabilidades esperadas de sucesso. Com todos os modelos detectamos diminuição na probabilidade esperada de sucesso para indivíduos em estágio inicial da doença e maior redução para os em estágio avançado $\left(\beta_{\mu 2}<\beta_{\mu 1}<0\right)$. Detectamos, também, diminuição na probabilidade esperada sob a condição dificultadora $\left(\beta_{\mu D}<0\right)$ e também quando os indivíduos estão realizando a sequência controle $\left(\beta_{\mu C}<0\right)$. Porém, apenas sob o modelo Beta-Binomial/PoissonGama com enlace gerado pelas probabilidades esperadas de sucesso foi possível detectar 
Tabela 3.4: Estimativas dos valores esperados de $\lambda\left(\mathbf{z}_{\lambda g h}\right)$

\begin{tabular}{|c|c|c|c|c|c|}
\hline $\begin{array}{c}\text { Estágio } \\
\text { da doença }\end{array}$ & Avaliação & Mão & Seqüência & $\begin{array}{c}\text { Valor } \\
\text { esperado }\end{array}$ & $\begin{array}{c}\text { Erro } \\
\text { padrão }\end{array}$ \\
\hline controle & final & preferencial & treinada & 12,0 & 0,5 \\
\hline controle & final & preferencial & controle & 9,4 & 0,4 \\
\hline controle & final & não & treinada & 25,5 & 1,0 \\
\hline controle & final & não & controle & 19,9 & 0,8 \\
\hline controle & dificultadora & preferencial & treinada & 11,2 & 0,5 \\
\hline controle & dificultadora & preferencial & controle & 8,7 & 0,4 \\
\hline controle & dificultadora & não & treinada & 23,5 & 0,9 \\
\hline controle & dificultadora & não & controle & 18,3 & 0,8 \\
\hline inicial & final & preferencial & treinada & 54,0 & 2,8 \\
\hline inicial & final & preferencial & controle & 42,0 & 2,2 \\
\hline inicial & final & não & treinada & 113,6 & 5,6 \\
\hline inicial & final & não & controle & 88,5 & 4,4 \\
\hline inicial & dificultadora & preferencial & treinada & 49,8 & 2,6 \\
\hline inicial & dificultadora & preferencial & controle & 38,8 & 2,1 \\
\hline inicial & dificultadora & não & treinada & 104,8 & 5,2 \\
\hline inicial & dificultadora & não & controle & 81,6 & 4,1 \\
\hline avançado & final & preferencial & treinada & 20,6 & 1,1 \\
\hline avançado & final & preferencial & controle & 16,1 & 0,9 \\
\hline avançado & final & não & treinada & 43,4 & 2,5 \\
\hline avançado & final & não & controle & 33,8 & 2,0 \\
\hline avançado & dificultadora & preferencial & treinada & 19,0 & 1,0 \\
\hline avançado & dificultadora & preferencial & controle & 14,8 & 0,8 \\
\hline avançado & dificultadora & não & treinada & 40,1 & 2,3 \\
\hline avançado & dificultadora & não & controle & 31,2 & 1,8 \\
\hline
\end{tabular}


Tabela 3.5: Estimativas dos valores esperados de $\eta_{1}\left(\mathbf{z}_{\eta_{1} g}\right)$

\begin{tabular}{cccc}
\hline $\begin{array}{c}\text { Estágio } \\
\text { da doença }\end{array}$ & Mão & $\begin{array}{c}\text { Valor } \\
\text { esperado }\end{array}$ & $\begin{array}{c}\text { Erro } \\
\text { padrão }\end{array}$ \\
\hline controle & preferencial & 0,57 & 0,04 \\
controle & não & 0,17 & 0,01 \\
\hline inicial & preferencial & $-1,19$ & 0,15 \\
inicial & não & $-1,59$ & 0,20 \\
\hline avançado & preferencial & $-0,78$ & 0,20 \\
avançado & não & $-1,19$ & 0,30 \\
\hline
\end{tabular}

Tabela 3.6: Estimativas dos valores esperados de $\delta\left(\mathbf{z}_{\delta g}\right)$

\begin{tabular}{cccc}
\hline $\begin{array}{c}\text { Estágio } \\
\text { da doença }\end{array}$ & Mão & $\begin{array}{c}\text { Valor } \\
\text { esperado }\end{array}$ & $\begin{array}{c}\text { Erro } \\
\text { padrão }\end{array}$ \\
\hline controle ou avançado & preferencial & 0,22 & 0,08 \\
controle ou avançado & não & 0,03 & 0,02 \\
\hline inicial & preferencial & 0,02 & 0,01 \\
inicial & não & 0,04 & 0,02 \\
\hline
\end{tabular}

uma diminuição ainda maior para a probabilidade de sucesso esperada sob a condição dificultadora para pacientes em estágio inicial da doença $\left(\beta_{\mu(1 * D)}<0\right)$; e, por outro lado, detectar que tal probabilidade é um pouco maior para participantes usando a mão não preferencial $\left(\beta_{\mu(D * N)}>0\right)$ ou realizando a seqüência controle $\left(\beta_{\mu(D * C)}>0\right)$.

De um modo geral, o modelo Beta-Binomial/Poisson-Gama com enlace gerado pelas probabilidades esperadas de sucesso foi o que apresentou maiores erros padrões para as estimativas entre os três modelos. Tais erros padrões maiores podem ser justificados pela complexidade do modelo que incorpora o efeito da sobredispersão nesses erros padrões. Além disso, comparando as estimativas dos valores esperados para acertos e totais de tentativas podemos dizer que na maioria dos casos os três modelos geraram estimativas semelhantes. O número esperado de acertos e do total de tentativas para indivíduos em estágio avançado da doença, na avaliação final, realizando a sequência treinada com a mão não preferencial foram subestimados pelos três modelos (da ordem de 30\%); o que mais se aproximou foi o Beta-Binomial/Poisson-Gama com enlace gerado pelas probabilidades 
Tabela 3.7: Estimativas dos valores esperados do número de acertos e total de tentativas, e respectivos erros padrões (entre parênteses)

\begin{tabular}{cccccc}
\hline $\begin{array}{c}\text { Estágio } \\
\text { da doença }\end{array}$ & Avaliação & Mão & Seqüência & Acertos & Totais \\
\hline controle & final & preferencial & treinada & $31,2(3,1)$ & $32,2(3,3)$ \\
controle & final & preferencial & controle & $20,8(2,1)$ & $25,1(2,6)$ \\
controle & final & não & treinada & $34,3(2,2)$ & $35,5(2,0)$ \\
controle & final & não & controle & $22,9(1,7)$ & $27,6(1,6)$ \\
controle & dificultadora & preferencial & treinada & $25,7(2,6)$ & $29,7(3,0)$ \\
controle & dificultadora & preferencial & controle & $17,0(1,8)$ & $23,2(2,4)$ \\
controle & dificultadora & não & treinada & $29,8(2,0)$ & $32,7(1,9)$ \\
controle & dificultadora & não & controle & $20,8(1,5)$ & $25,5(1,5)$ \\
\hline inicial & final & preferencial & treinada & $21,1(1,9)$ & $22,2(1,9)$ \\
inicial & final & preferencial & controle & $12,9(1,2)$ & $17,3(1,5)$ \\
inicial & final & não & treinada & $21,3(2,7)$ & $22,4(2,7)$ \\
inicial & final & não & controle & $13,1(1,7)$ & $17,5(2,1)$ \\
inicial & dificultadora & preferencial & treinada & $12,6(1,2)$ & $20,1(1,8)$ \\
inicial & dificultadora & preferencial & controle & $6,5(0,7)$ & $16,0(1,4)$ \\
inicial & dificultadora & não & treinada & $14,9(1,9)$ & $20,7(2,5)$ \\
inicial & dificultadora & não & controle & $8,4(1,2)$ & $16,1(2,0)$ \\
\hline avançado & final & preferencial & treinada & $13,6(1,4)$ & $15,7(1,5)$ \\
avançado & final & preferencial & controle & $6,3(0,9)$ & $12,2(1,2)$ \\
avançado & final & não & treinada & $18,6(2,9)$ & $21,5(3,1)$ \\
avançado & final & não & controle & $8,7(1,5)$ & $16,7(2,5)$ \\
avançado & dificultadora & preferencial & treinada & $8,5(1,1)$ & $14,4(1,4)$ \\
avançado & dificultadora & preferencial & controle & $4,2(0,9)$ & $11,3(1,1)$ \\
avançado & dificultadora & não & treinada & $13,8(2,2)$ & $19,8(2,9)$ \\
avançado & dificultadora & não & controle & $7,6(1,3)$ & $15,4(2,3)$ \\
\hline
\end{tabular}


esperadas de sucesso (da ordem de 10\%).

Considerando apenas o modelo Beta-Binomial/Poisson-Gama com enlace gerado pelas probabilidades esperadas de sucesso, para o parâmetro relacionado a dispersão do número de acertos, pode-se concluir que seu valor esperado é menor para participantes em estágio inicial da doença $\left(\beta_{\theta 1}<0\right)$ e maior para aqueles em estágio avançado $\left(\beta_{\theta 2}>0\right)$, quando comparados com o grupo controle. Além disso, sob a condição dificultadora, o valor esperado de $\theta\left(\mathbf{z}_{\theta g h}\right)$ é maior do que na avaliação final $\left(\beta_{\theta D}<0\right)$; e usando a mão não preferencial tal valor diminui se comparado àquele usando a mão preferencial $\left(\beta_{\theta N}<0\right)$. Por fim, tais valores esperados diminuem sob a condição dificultadora, quando se trata de participantes em estágio avançado da doença $\left(\beta_{\theta(2 * D)}<0\right)$ ou usando a mão não preferencial $\left(\beta_{\theta(D * N)}<0\right)$; e, por outro lado, aumentam quando se trata de participantes em estágio inicial da doença usando a mão não preferencial $\left(\beta_{\theta(1 * N)}>0\right)$. Para o parâmetro relacionado à sobredispersão do total de tentativas, $\delta\left(\mathbf{z}_{\delta g}\right)$, podemos concluir que não há diferença entre participantes do grupo controle e dos em estágio avançado da doença $\left(\beta_{\delta 2}=0\right)$, mas os em estágio inicial têm valor esperado menor $\left(\beta_{\delta 1}<0\right)$. Porém quando se trata de pacientes em estágio inicial da doença usando a mão não preferencial, tal valor esperado aumenta $\left(\beta_{\delta(1 * N)}>0\right)$.

Por fim, considerando os valores esperados de $\eta_{1}\left(\mathbf{z}_{\eta_{1} g}\right)$, parâmetro que modela a relação entre o total de tentativas e a média da probabilidade esperada de acertos nos 4 instantes de observação, presente apenas no modelo Beta-Binomial/Poisson-Gama com enlace gerado pelas probabilidades esperadas de sucesso, podemos concluir que para o grupo controle usando a mão preferencial tal relação é positiva $\left(\beta_{\eta_{1} 0}>0\right)$, ou seja, quanto maior tal probabilidade média, maior o número de tentativas. Já para os participantes em estágio avançado da doença essa relação se torna negativa $\left(\beta_{\eta_{1} 0}+\beta_{\eta_{1} 2}<0\right)$ e menor ainda para pacientes em estágio inicial $\left(\beta_{\eta_{1} 1}<\beta_{\eta_{1} 2}\right)$. Os participantes que usam a mão não preferencial tem essa relação entre a probabilidade esperada de sucesso e o total de tentativas esperado menor do que aqueles que usam a mão preferencial $\left(\beta_{\eta_{1} N}<0\right)$, mas essa relação ainda é positiva para participantes controles $\left(\beta_{\eta_{1} 0}+\beta_{\eta_{1} N}>0\right)$. 


\subsection{Modelo Beta-Binomial/Poisson-Gama com enlace gerado pelas probabilidades individuais de sucesso}

Uma generalização do modelo apresentado na seção 3.1 corresponde ao acréscimo de

$$
\lambda_{g h}=\exp \left\{\eta_{0}\left(\mathbf{z}_{\eta_{0} g h}\right)+\eta_{1}\left(\mathbf{z}_{\eta_{1} g h}\right) \log \left(\frac{\mu\left(\mathbf{z}_{\mu g h}\right)}{1-\mu\left(\mathbf{z}_{\mu g h}\right)}\right)\right\}
$$

a (1.2), (1.3), (2.1) e (2.2), ou seja, ao invés de modelar $\alpha_{g}$ em função de $\bar{\mu}_{g}$ (média dos $p$ valores de $\mu\left(\mathbf{z}_{\mu g h}\right)$ do $g$-ésimo indivíduo), modelamos $\lambda_{g h}$ em função de $\mu\left(\mathbf{z}_{\mu g h}\right)$. Dessa maneira poderíamos comparar os parâmetros $\eta_{1}\left(\mathbf{z}_{\eta_{1} g h}\right)$ correspondentes aos diferentes instantes de avaliação, o que tornaria o modelo mais genérico. A estimação dos parâmetros desse modelo não é viável usando o processo iterativo de Newton-Raphson, já que primeiras derivadas em relação a $\eta_{0}$ e em relação a $\eta_{1}$ geram funções linearmente dependentes. Para contornar essa indeterminação e permitir a comparação do parâmetro de enlace nos diferentes instantes de avaliação, consideramos (1.2), (1.2), (2.1), (2.2) e acrescentamos

$$
\lambda_{g h}=\exp \left\{\eta_{0}\left(\mathbf{z}_{\eta_{0} g h}\right)+\eta_{1}\left(\mathbf{z}_{\eta_{1} g h}\right) \log \left(\frac{\pi_{g h}}{1-\pi_{g h}}\right)\right\}
$$

em que $\mathbf{z}_{\eta 0 g h}$ e $\mathbf{z}_{\eta 1 g h}$ são vetores de covariáveis.

O parâmetro $\eta_{1}\left(\mathbf{z}_{\eta_{1} g h}\right)$ em (3.5), modela a relação entre a probabilidade individual de acertos $\left(\pi_{g h}\right)$ e um dos termos do valor esperado do total de tentativas $\left(\lambda_{g h}\right)$. A interpretação desse parâmetro é semelhante à apresentada na seção 3.1: quando $\eta_{1}\left(\mathbf{z}_{\eta_{1} g h}\right)>0$, quanto maior a probabilidade de acerto, maior o valor esperado do total de tentativas; ocorrendo o contrário quando $\eta_{1}\left(\mathbf{z}_{\eta_{1} g h}\right)<0$; se $\eta_{1}\left(\mathbf{z}_{\eta_{1} g h}\right)=0$, não há relação entre a probabilidade de acerto e o valor esperado do total de tentativas e o modelo acima recai naquele apresentado no capítulo 2.

Com base em (1.2), (1.3), (2.1) e (2.2), os momentos centrais e as covariâncias,

se escritos em função de $\lambda_{g h}$, são iguais aos apresentados no capítulo 2; além disso, a distribuição conjunta das contagens de acertos e totais das duas seqüências também é equivalente a apresentada no capítulo 2. Porém, semelhante ao que aconteceu com o modelo Beta-Binomial/Poisson-Gama com enlace gerado pelas probabilidades esperadas de 
sucesso, como o valor de $\lambda_{g h}$ depende do valor de $\pi_{g h}$, a estimação dos parâmetros do modelo Poisson-Gama não pode mais ser feita separadamente da estimação dos parâmetros do modelo Beta-Binomial.

Para investigar os efeitos das covariáveis, adotamos os modelos log-lineares (1.15), (1.16), (2.13), (2.14) e

$$
\begin{aligned}
& \eta_{0}\left(\mathbf{z}_{\eta_{0} g h}\right)=\mathbf{z}_{\eta_{0} g h}^{\prime} \boldsymbol{\beta}_{\eta_{0}} \\
& \eta_{1}\left(\mathbf{z}_{\eta_{1} g h}\right)=\mathbf{z}_{\eta_{1} g h}^{\prime} \boldsymbol{\beta}_{\eta_{1}}
\end{aligned}
$$

em que $\boldsymbol{\beta}_{\eta_{0}}$ e $\boldsymbol{\beta}_{\eta_{1}}$ são vetores de parâmetros a serem estimados.

De (1.2), (1.3), (2.1), (2.2) e (3.5), a distribuição conjunta de $\left(X_{g 1}, N_{g 1}, \ldots, X_{g p}, N_{g p}\right)$ pode ser escrita como

$$
\begin{aligned}
& f\left(x_{g 1}, n_{g 1}, \ldots, x_{g p}, n_{g p}\right)=\int_{0}^{\infty}\left\{\prod _ { h = 1 } ^ { p } \left[\int_{0}^{1}\left(\begin{array}{c}
n_{g h} \\
x_{g h}
\end{array}\right) \pi_{g h}^{x_{g h}}\left(1-\pi_{g h}\right)^{n_{g h}-x_{g h}} \Gamma\left(\frac{1}{\theta\left(\mathbf{z}_{\theta g h}\right)}\right)\right.\right. \\
& \left(\Gamma\left(\frac{\mu\left(\mathbf{z}_{\mu g h}\right)}{\theta\left(\mathbf{z}_{\theta g h}\right)}\right) \Gamma\left(\frac{1-\mu\left(\mathbf{z}_{\mu g h}\right)}{\theta\left(\mathbf{z}_{\theta g h}\right)}\right)\right)^{-1} \pi_{g h}^{\mu\left(\mathbf{z}_{\mu g h}\right) / \theta\left(\mathbf{z}_{\theta g h}\right)}\left(1-\pi_{g h}\right)^{\left(1-\mu\left(\mathbf{z}_{\mu g h}\right)\right) / \theta\left(\mathbf{z}_{\theta g h}\right)} \\
& \left.\left.\frac{e^{-\lambda_{g h} \tau_{g}} \lambda_{g h}^{n_{g h}} \tau_{g}^{n_{g h}}}{n_{g h} !} d \pi_{g h}\right] \frac{\tau_{g}^{\alpha\left(\mathbf{z}_{\alpha g}\right) / \delta\left(\mathbf{z}_{\delta g}\right)-1} e^{-\tau_{g} / \delta\left(\mathbf{z}_{\delta g}\right)}}{\delta\left(\mathbf{z}_{\delta g}\right)^{\alpha\left(\mathbf{z}_{\alpha g}\right) / \delta\left(\mathbf{z}_{\delta g}\right)}}\left(\Gamma\left(\frac{\alpha\left(\mathbf{z}_{\alpha g}\right)}{\delta\left(\mathbf{z}_{\delta g}\right)}\right)\right)^{-1}\right\} d \tau_{g}
\end{aligned}
$$

Como não há forma fechada para as integrais em relação a $\pi_{g h}$ (já que $\lambda_{g h}$ é função de $\pi_{g h}$ ), necessita-se usar algum método iterativo para estimar os parâmetros do modelo. Considerando as variáveis $\lambda_{g h}$ e $\tau_{g}$ como latentes, tal estimação pode ser feita usando o algoritmo EM (Dempster, Laird e Rubin, 1977).

O núcleo da função log-verossimilhança aumentada (incluindo as variáveis observadas e as latentes) é dado por

$$
\begin{aligned}
L \propto & \sum_{g=1}^{m}\left\{\sum _ { h = 1 } ^ { p } \left[\log \Gamma\left(\frac{1}{\theta\left(\mathbf{z}_{\theta g h}\right)}\right)-\log \Gamma\left(\frac{\mu\left(\mathbf{z}_{\mu g h}\right)}{\theta\left(\mathbf{z}_{\theta g h}\right)}\right)-\log \Gamma\left(\frac{1-\mu\left(\mathbf{z}_{\mu g h}\right)}{\theta\left(\mathbf{z}_{\theta g h}\right)}\right)\right.\right. \\
& +\frac{\mu\left(\mathbf{z}_{\mu g h}\right)}{\theta\left(\mathbf{z}_{\theta g h}\right)} \log \pi_{g h}+\frac{\left(1-\mu\left(\mathbf{z}_{\mu g h}\right)\right.}{\theta\left(\mathbf{z}_{\theta g h}\right)} \log \left(1-\pi_{g h}\right)-\tau_{g} e^{\eta_{0}\left(\mathbf{z}_{\eta_{0} g h}\right)}\left(\frac{\pi_{g h}}{1-\pi_{g h}}\right)^{\eta_{1}\left(\mathbf{z}_{\eta_{1} g h}\right)} \\
& \left.+n_{g h} \eta_{0}\left(\mathbf{z}_{\eta_{0} g h}\right)+n_{g h} \eta_{1}\left(\mathbf{z}_{\eta_{1} g h}\right) \log \left(\pi_{g h}\right)-n_{g h} \eta_{1}\left(\mathbf{z}_{\eta_{1} g h}\right) \log \left(1-\pi_{g h}\right)\right] \\
& \left.-\log \Gamma\left(\frac{\alpha\left(\mathbf{z}_{\alpha g h}\right)}{\delta\left(\mathbf{z}_{\delta g h}\right)}\right)-\frac{\alpha\left(\mathbf{z}_{\alpha g h}\right)}{\delta\left(\mathbf{z}_{\delta g h}\right)} \log \delta\left(\mathbf{z}_{\delta g h}\right)+\frac{\alpha\left(\mathbf{z}_{\alpha g h}\right)}{\delta\left(\mathbf{z}_{\delta g h}\right)} \log \tau_{g}-\frac{\tau_{g}}{\delta\left(\mathbf{z}_{\delta g h}\right)}\right\}
\end{aligned}
$$


Para o passo E, a esperança de (3.9) dados os valores observados $\mathbf{x}=\left(x_{11}, \ldots, x_{g h}, \ldots, x_{m p}\right)$ e $\mathbf{n}=\left(n_{11}, \ldots, n_{g h}, \ldots, n_{m p}\right)$, e o vetor de parâmetros $\boldsymbol{\beta}=\left(\boldsymbol{\beta}_{\mu}, \boldsymbol{\beta}_{\theta}, \boldsymbol{\beta}_{\eta_{0}}, \boldsymbol{\beta}_{\eta_{1}}, \boldsymbol{\beta}_{\alpha}, \boldsymbol{\beta}_{\delta}\right)$ é

$$
\begin{aligned}
Q= & \sum_{g=1}^{m}\left\{\sum _ { h = 1 } ^ { p } \left[\log \Gamma\left(\frac{1}{\theta\left(\mathbf{z}_{\theta g h}\right)}\right)-\log \Gamma\left(\frac{\mu\left(\mathbf{z}_{\mu g h}\right)}{\theta\left(\mathbf{z}_{\theta g h}\right)}\right)-\log \Gamma\left(\frac{1-\mu\left(\mathbf{z}_{\mu g h}\right)}{\theta\left(\mathbf{z}_{\theta g h}\right)}\right)\right.\right. \\
& +\frac{\mu\left(\mathbf{z}_{\mu g h}\right)}{\theta\left(\mathbf{z}_{\theta g h}\right)} p_{g h}^{(j)}+\frac{\left(1-\mu\left(\mathbf{z}_{\mu g h}\right)\right.}{\theta\left(\mathbf{z}_{\theta g h}\right)} q_{g h}^{(j)}-r_{g h}^{(j)} \eta_{1}\left(\mathbf{z}_{\eta_{1} g h}\right) e^{\eta_{0}\left(\mathbf{z}_{\eta_{0} g h}\right)}+n_{g h} \eta_{0}\left(\mathbf{z}_{\eta_{0} g h}\right) \\
& \left.+n_{g h} \eta_{1}\left(\mathbf{z}_{\eta_{1} g h}\right) p_{g h}^{(j)}-n_{g h} \eta_{1}\left(\mathbf{z}_{\eta_{1} g h}\right) q_{g h}^{(j)}\right]-\log \Gamma\left(\frac{\alpha\left(\mathbf{z}_{\alpha g h}\right)}{\delta\left(\mathbf{z}_{\delta g h}\right)}\right) \\
& \left.-\frac{\alpha\left(\mathbf{z}_{\alpha g h}\right)}{\delta\left(\mathbf{z}_{\delta g h}\right)} \log \delta\left(\mathbf{z}_{\delta g h}\right)+\frac{\alpha\left(\mathbf{z}_{\alpha g h}\right)}{\delta\left(\mathbf{z}_{\delta g h}\right)} s_{g}^{(j)}-\frac{w_{g}^{(j)}}{\delta\left(\mathbf{z}_{\delta g h}\right)}\right\}
\end{aligned}
$$

com

$$
\begin{aligned}
p_{g h}^{(j)} & =E\left[\log \left(\pi_{g h}\right) \mid \mathbf{x}, \mathbf{n}, \boldsymbol{\beta}^{(j)}\right] \\
q_{g h}^{(j)} & =E\left[\log \left(1-\pi_{g h}\right) \mid \mathbf{x}, \mathbf{n}, \boldsymbol{\beta}^{(j)}\right] \\
r_{g h}^{(j)}\left(\eta_{1}\right) & =E\left[\tau_{g} \log \left(\frac{\pi_{g h}}{1-\pi_{g h}}\right)^{\eta_{1}\left(\mathbf{z}_{\eta_{1} g h}\right)} \mid \mathbf{x}, \mathbf{n}, \boldsymbol{\beta}^{(j)}\right] \\
s_{g}^{(j)} & =E\left[\log \left(\tau_{g}\right) \mid \mathbf{x}, \mathbf{n}, \boldsymbol{\beta}^{(j)}\right] \\
w_{g}^{(j)} & =E\left[\tau_{g} \mid \mathbf{x}, \mathbf{n}, \boldsymbol{\beta}^{(j)}\right]
\end{aligned}
$$

Fazendo $\boldsymbol{\pi}=\left(\pi_{g 1}, \ldots, \pi_{g h}, \ldots, \pi_{g p}\right)$, as esperanças condicionais (3.11), (3.12), (3.13), (3.14) e (3.15) podem ser calculadas como

$$
\begin{aligned}
& E\left[g\left(\pi_{g h}, \tau_{g}\right) \mid \mathbf{x}, \mathbf{n}, \boldsymbol{\beta}^{(j)}\right]=\int_{0}^{\infty} \prod_{h=1}^{p}\left[\int_{0}^{1} g\left(\pi_{g h}, \tau_{g}\right) f\left(\boldsymbol{\pi}, \tau_{g} \mid \mathbf{x}, \mathbf{n}, \boldsymbol{\beta}^{(j)}\right) d \pi_{g h}\right] d \tau_{g} \\
& =\frac{\int_{0}^{\infty} \prod_{h=1}^{p}\left[\int_{0}^{1} g\left(\pi_{g h}, \tau_{g}\right) f\left(\boldsymbol{\pi}, \tau_{g}, \mathbf{x}, \mathbf{n} \mid \boldsymbol{\beta}^{(j)}\right) d \pi_{g h}\right] d \tau_{g}}{\int_{0}^{\infty} \prod_{h=1}^{p}\left[\int_{0}^{1} f\left(\boldsymbol{\pi}, \tau_{g}, \mathbf{x}, \mathbf{n} \mid \boldsymbol{\beta}^{(j)}\right) d \pi_{g h}\right] d \tau_{g}} \\
& =\frac{\int_{0}^{\infty} \prod_{h=1}^{p}\left[\int_{0}^{1} g\left(\pi_{g h}, \tau_{g}\right) f\left(\mathbf{x} \mid \boldsymbol{\pi}, \mathbf{n}, \boldsymbol{\beta}^{(j)}\right) f\left(\boldsymbol{\pi} \mid \boldsymbol{\beta}^{(j)}\right) f\left(\mathbf{n} \mid \tau_{g}, \boldsymbol{\beta}^{(j)}\right) f\left(\tau_{g} \mid \boldsymbol{\beta}^{(j)}\right) d \pi_{g h}\right] d \tau_{g}}{\int_{0}^{\infty} \prod_{h=1}^{p}\left[\int_{0}^{1} f\left(\mathbf{x} \mid \boldsymbol{\pi}, \mathbf{n}, \boldsymbol{\beta}^{(j)}\right) f\left(\boldsymbol{\pi} \mid \boldsymbol{\beta}^{(j)}\right) f\left(\mathbf{n} \mid \tau_{g}, \boldsymbol{\beta}^{(j)}\right) f\left(\tau_{g} \mid \boldsymbol{\beta}^{(j)}\right) d \pi_{g h}\right] d \tau_{g}}
\end{aligned}
$$

Tais esperanças, $E\left[g\left(\pi_{g h}, \tau_{g}\right) \mid \mathbf{x}, \mathbf{n}, \boldsymbol{\beta}^{(j)}\right]$, podem ser estimadas usando o método de Monte Carlo por meio da relação

$$
E\left[g\left(\pi_{g h}, \tau_{g}\right) \mid \mathbf{x}, \mathbf{n}, \boldsymbol{\beta}^{(j)}\right] \approx \frac{\sum_{k=1}^{K} \prod_{h=1}^{p}\left[\sum_{i=1}^{I} g\left(\pi_{g h}^{(i)}, \tau_{g}^{(k)}\right) f\left(\mathbf{x} \mid \boldsymbol{\pi}^{(i)}, \mathbf{n}, \boldsymbol{\beta}^{(j)}\right) f\left(\mathbf{n} \mid \tau_{g}^{(k)}, \boldsymbol{\beta}^{(j)}\right)\right]}{\sum_{k=1}^{K} \prod_{h=1}^{p}\left[\sum_{i=1}^{I} f\left(\mathbf{x} \mid \boldsymbol{\pi}^{(i)}, \mathbf{n}, \boldsymbol{\beta}^{(j)}\right) f\left(\mathbf{n} \mid \tau_{g}^{(k)}, \boldsymbol{\beta}^{(j)}\right)\right]}
$$


$\operatorname{com} \pi_{g h}^{(i)}, i=1, \ldots, I$, gerados da distribuição Beta $\left(\mu\left(\mathbf{z}_{\mu g h}\right)^{(j)} / \theta\left(\mathbf{z}_{\theta g h}\right)^{(j)},\left[1-\mu\left(\mathbf{z}_{\mu g h}\right)^{(j)}\right] /\right.$ $\left.\theta\left(\mathbf{z}_{\theta g h}\right)^{(j)}\right)$ e $\tau_{g}^{k}, k=1, \ldots, K$, gerados da distribuição Gama $\left(\alpha\left(\mathbf{z}_{\alpha g}\right)^{(j)} / \delta\left(\mathbf{z}_{\delta g}\right), 1 / \delta\left(\mathbf{z}_{\delta g}\right)^{(j)}\right)$.

No passo M, para a maximização de (3.10), propomos um processo iterativo de Newton-Raphson, cujas derivadas estão apresentadas no Apêndice D. A partir da equação (3.10) é possível implementar o processo de maximização por meio de três processos iterativos separados: um para $\mu\left(\mathbf{z}_{\mu g h}\right)$ e $\theta\left(\mathbf{z}_{\theta g h}\right)$, outro para $\eta_{0}\left(\mathbf{z}_{\eta_{0} g h}\right)$ e $\eta_{1}\left(\mathbf{z}_{\eta_{1} g h}\right)$ e um último para $\alpha\left(\mathbf{z}_{\alpha g}\right)$ e $\delta\left(\mathbf{z}_{\delta g}\right)$. Tal separação é possível já que (3.10) pode ser escrita como $Q=f_{1}\left(\boldsymbol{\beta}_{\mu}, \boldsymbol{\beta}_{\theta}\right)+f_{2}\left(\boldsymbol{\beta}_{\eta_{0}}, \boldsymbol{\beta}_{\eta_{1}}\right)+f_{3}\left(\boldsymbol{\beta}_{\alpha}, \boldsymbol{\beta}_{\delta}\right)$. Os resultados obtidos com o modelo BetaBinomial/Poisson-Gama do capítulo anterior podem ser usados como valores iniciais para o processo iterativo, considerando os valores de $\boldsymbol{\beta}_{\lambda}$ para $\boldsymbol{\beta}_{\eta_{0}}$ e zero para $\boldsymbol{\beta}_{\eta_{1}}$; como na seção 3.1 , o valor zero pode ser usado para $\boldsymbol{\beta}_{\eta_{1}}$ para manter o processo iterativo estável. Testes da razão de verossimilhanças podem ser usados para redução do modelo. O processo iterativo foi implementado nos softwares R e Ox e os códigos correspondentes podem ser obtidos em http://www.ime.usp.br/ jmsinger.

\subsection{Análise do exemplo por meio do modelo Beta- Binomial/Poisson-Gama com enlace gerado pelas probabilidades individuais de sucesso}

Para analisar o exemplo, ajustamos modelos log-lineares semelhantes aos apresentados nas seções 1.1, 2.2 e 3.1, e usamos as mesmas estratégias de eliminação dos termos não significativos. A indexação dos parâmetros e a cela de referência usadas também são as mesmas apresentadas nas seções 1.1, 2.2 e 3.1.

O vetor de parâmetros inicial é $\boldsymbol{\beta}=\left(\boldsymbol{\beta}_{\mu}, \boldsymbol{\beta}_{\theta}, \boldsymbol{\beta}_{\eta_{0}}, \boldsymbol{\beta}_{\eta_{1}}, \boldsymbol{\beta}_{\alpha}, \boldsymbol{\beta}_{\delta}\right)$ em que

$$
\begin{aligned}
\boldsymbol{\beta}_{m}= & \left(\beta_{m 0}, \beta_{m 1}, \beta_{m 2}, \beta_{m N}, \beta_{m D}, \beta_{m C},\right. \\
& \left.\beta_{m(1 * D)}, \beta_{m(1 * N)}, \beta_{m(1 * C)}, \beta_{m(2 * D)}, \beta_{m(2 * N)}, \beta_{m(2 * C)}, \beta_{m(D * N)}, \beta_{m(D * C)}, \beta_{m(N * C)}\right) \\
\boldsymbol{\beta}_{n}= & \left(\beta_{n 0}, \beta_{n 1}, \beta_{n 2}, \beta_{n N}, \beta_{n(1 * N)}, \beta_{n(2 * N)}\right)
\end{aligned}
$$

$\operatorname{com} m=\mu, \theta, \eta_{0}, \eta_{1}$ e $n=\alpha, \delta$. 
Como nos modelos apresentados anteriormente, o processo iterativo proposto mostrou-se sensível aos valores iniciais, especialmente para as interações. Usamos a mesma estratégia descrita na seção 1.1. para o ajuste do modelo inicial. Além disso, devido a limitações de arredondamento do "software" usado, tomamos: $10^{-16} \leq \pi_{g h}^{(i)} \leq 1-10^{-16} \mathrm{e}$ $\tau_{g}^{(k)} \geq 10^{-16}$.

Como sugerido por Wei e Tanner (1990), começamos o processo iterativo com $I$ e $K$ menores e depois aumentamos tais valores para obter convergências de ordens melhores. Com $I=K=1000$, obtivemos uma convergência com aproximação da ordem de $10^{-1}$; para obter aproximação de $10^{-2}$, foram necessários $I=K=5000$ para o modelo contendo apenas efeitos principais, e $I=K=15000$ para a inclusão das interações de primeira ordem. Como o modelo apresenta muitos parâmetros a serem estimados, o processo se tornou computacionalmente muito lento, inviabilizando o aumento de $I$ e $K$ até obtermos uma convergência melhor.

As estimativas dos parâmetros do modelo inicial (com respectivos erros padrões) estão apresentadas nas Tabelas 3.8, 3.9 e 3.10. Apesar de termos obtido apenas uma convergência com aproximação da ordem de $10^{-2}$, tentamos reduzir o modelo inicial retirando os parâmetros identificados como não significativos. Para comparar possíveis modelos reduzidos com o inicial foram usados testes da razão de verossimilhanças. Porém, tais testes se mostraram muito instáveis já que também é necessário estimar a função logverossimilhança [obtida a partir de (3.8)] via Monte Carlo, impossibilitando a comparação do modelo inicial com subseqüentes modelos reduzidos.

Como não obtivemos convergência dentro dos limites de aproximação desejados $(\epsilon<$ $10^{-5}$ ) e por não termos obtido um modelo reduzido final, não estimamos os parâmetros $\mu\left(\mathbf{z}_{\mu g h}\right), \theta\left(\mathbf{z}_{\theta g h}\right), \eta_{0}\left(\mathbf{z}_{\eta_{0} g h}\right), \eta_{1}\left(\mathbf{z}_{\eta_{1} g h}\right), \alpha\left(\mathbf{z}_{\alpha g}\right)$ e $\delta\left(\mathbf{z}_{\delta g}\right)$. Dessa maneira, sob este modelo, torna-se inviável a conclusão sobre diferenças detectadas entre os grupos de participantes e instantes de observação, e consequentemente a comparação dos resultados obtivos sob este modelo com os obtidos sob os demais modelos anteriores. Porém, descritivamente, podemos notar que as estimativas dos parâmetros obtidas são muito semelhantes às dos outros modelos; como menor probabilidade e total de tentativas esperados para pacientes em estágio inicial e avançado quando comparados com os participantes controles, ou 
mesmo a diminuição desses valores esperados para a avaliação sob a condição dificultadora se comparada com a final.

\subsection{Aspectos computacionais}

O modelo implementado no software Ox se mostrou mais rápido do que o implementado no $\mathrm{R}$ (cerca de $70 \%$ do tempo). Como o valor das primeiras derivadas calculadas com os parâmetros estimados foram se mostrando cada vez mais próximas de zero, conforme aumentávamos $I$ e $K$ e realizávamos mais iterações, acreditamos que o modelo se tornará viável com computadores mais velozes. Além disso, todas as derivadas do modelo para o processo iterativo de Newton-Raphson foram conferidas com o software Maple.

Por fim, outra evidência baseada em simulações mostra que o modelo faz sentido e que sua única restrição é computacional. Usando o modelo para exemplos simulados com $p<4$, o algoritmo de Newton-Raphson se mostrou mais rápido e converigiu mais rapidamente. Por exemplo, para uma situação simulada com $p=1$ e apenas dois grupos de participantes, foram necessárias mais de 1000 iterações e $I=10000$ para obtermos uma convergência com aproximação da ordem de $10^{-4}$. Essa situação com $p=1$ reflete exatamente a apresentada por Zhu et al. (2003), que apenas citam que foi necessário um valor muito grande para o número de dados gerados para a técnica de Monte Carlo (denominado aqui por $I$ ). 
Tabela 3.8: Estimativas dos parâmetros $\boldsymbol{\beta}_{\mu}$ e $\boldsymbol{\beta}_{\theta}$ sob o modelo Beta-Binomial/PoissonGama com enlace gerado pelas probabilidades individuais de sucesso inicial e erros padrões

\begin{tabular}{|c|c|c|c|}
\hline Parâmetro & Relacionado ao & Estimativa & Erro Padrão \\
\hline$\beta_{\mu 0}$ & grupo controle, mão pref., aval. final e seq. treinada & 3,58 & 0,53 \\
\hline$\beta_{\mu 1}$ & efeito do estágio inicial & $-0,74$ & 0,58 \\
\hline$\beta_{\mu 2}$ & efeito do estágio avançado & $-1,79$ & 0,58 \\
\hline$\beta_{\mu D}$ & efeito da condição dificultadora & $-1,46$ & 0,47 \\
\hline$\beta_{\mu N}$ & efeito da mão não preferencial & $-0,14$ & 0,51 \\
\hline$\beta_{\mu C}$ & efeito da seqüência controle & $-1,85$ & 0,46 \\
\hline$\beta_{\mu(1 * D)}$ & efeito do estágio inicial e condição dificultadora & $-1,00$ & 0,46 \\
\hline$\beta_{\mu(1 * N)}$ & efeito do estágio inicial e mão não preferencial & 0,10 & 0,48 \\
\hline$\beta_{\mu(1 * C)}$ & efeito do estágio inicial e seqüência controle & 0,22 & 0,49 \\
\hline$\beta_{\mu(2 * D)}$ & efeito do estágio avançado e condição dificultadora & $-0,23$ & 0,45 \\
\hline$\beta_{\mu(2 * N)}$ & efeito do estágio avançado e mão não preferencial & 0,59 & 0,47 \\
\hline$\beta_{\mu(2 * C)}$ & efeito do estágio avançado e seqüência controle & $-0,03$ & 0,44 \\
\hline$\beta_{\mu(D * N)}$ & efeito da condição dificultadora e mão não preferencial & 0,43 & 0,37 \\
\hline$\beta_{\mu(D * C)}$ & efeito da condição dificultadora e sequência controle & 1,00 & 0,38 \\
\hline$\beta_{\mu(N * C)}$ & efeito da mão não preferencial e sequência controle & $-0,12$ & 0,39 \\
\hline$\beta_{\theta 0}$ & grupo controle, mão pref., aval. final e seq. treinada & $-1,38$ & 0,57 \\
\hline$\beta_{\theta 1}$ & efeito do estágio inicial & $-0,99$ & 0,64 \\
\hline$\beta_{\theta 2}$ & efeito do estágio avançado & 1,02 & 0,64 \\
\hline$\beta_{\theta D}$ & efeito da condição dificultadora & 2,02 & 0,49 \\
\hline$\beta_{\theta N}$ & efeito da mão não preferencial & $-1,58$ & 0,57 \\
\hline$\beta_{\theta C}$ & efeito da seqüência controle & 0,72 & 0,54 \\
\hline$\beta_{\theta(1 * D)}$ & efeito do estágio inicial e condição dificultadora & 0,64 & 0,51 \\
\hline$\beta_{\theta(1 * N)}$ & efeito do estágio inicial e mão não preferencial & 2,35 & 0,52 \\
\hline$\beta_{\theta(1 * C)}$ & efeito do estágio inicial e seqüência controle & $-0,37$ & 0,55 \\
\hline$\beta_{\theta(2 * D)}$ & efeito do estágio avançado e condição dificultadora & $-2,09$ & 0,52 \\
\hline$\beta_{\theta(2 * N)}$ & efeito do estágio avançado e mão não preferencial & $-0,04$ & 0,56 \\
\hline$\beta_{\theta(2 * C)}$ & efeito do estágio avançado e seqüência controle & 0,03 & 0,59 \\
\hline$\beta_{\theta(D * N)}$ & efeito da condição dificultadora e mão não preferencial & $-1,11$ & 0,43 \\
\hline$\beta_{\theta(D * C)}$ & efeito da condição dificultadora e sequência controle & $-0,08$ & 0,44 \\
\hline$\beta_{\theta(N * C)}$ & efeito da mão não preferencial e sequência controle & $-0,02$ & 0,44 \\
\hline
\end{tabular}

Códigos: pref.: preferencial, aval.: avaliação e seq.: seqüência 
Tabela 3.9: Estimativas dos parâmetros $\boldsymbol{\beta}_{\eta_{0}}$ e $\boldsymbol{\beta}_{\eta_{1}}$ sob o modelo Beta-Binomial/Poisson-Gama com enlace gerado pelas probabilidades individuais de sucesso inicial e erros padrões

\begin{tabular}{|c|c|c|c|}
\hline Parâmetro & Relacionado ao & Estimativa & Erro Padrão \\
\hline$\beta_{\eta_{0} 0}$ & grupo controle, mão pref., aval. final e seq. treinada & 2,62 & 0,08 \\
\hline$\beta_{\eta_{0} 1}$ & efeito do estágio inicial & $-0,62$ & 0,12 \\
\hline$\beta_{\eta_{0} 2}$ & efeito do estágio avançado & $-1,99$ & 0,12 \\
\hline$\beta_{\eta_{0} D}$ & efeito da condição dificultadora & $-0,17$ & 0,09 \\
\hline$\beta_{\eta_{0} N}$ & efeito da mão não preferencial & 0,03 & 0,10 \\
\hline$\beta_{\eta_{0} C}$ & efeito da seqüência controle & $-0,42$ & 0,09 \\
\hline$\beta_{\eta_{0}(1 * D)}$ & efeito do estágio inicial e condição dificultadora & 0,04 & 0,12 \\
\hline$\beta_{\eta_{0}(1 * N)}$ & efeito do estágio inicial e mão não preferencial & $-0,15$ & 0,11 \\
\hline$\beta_{\eta_{0}(1 * C)}$ & efeito do estágio inicial e seqüência controle & 0,30 & 0,10 \\
\hline$\beta_{\eta_{0}(2 * D)}$ & efeito do estágio avançado e condição dificultadora & 0,28 & 0,11 \\
\hline$\beta_{\eta_{0}(2 * N)}$ & efeito do estágio avançado e mão não preferencial & $-0,23$ & 0,12 \\
\hline$\beta_{\eta_{0}(2 * C)}$ & efeito do estágio avançado e seqüência controle & 0,31 & 0,12 \\
\hline$\beta_{\eta_{0}(D * N)}$ & efeito da condição dificultadora e mão não preferencial & 0,07 & 0,09 \\
\hline$\beta_{\eta_{0}(D * C)}$ & efeito da condição dificultadora e sequência controle & $-0,08$ & 0,08 \\
\hline$\beta_{\eta_{0}(N * C)}$ & efeito da mão não preferencial e sequência controle & 0,17 & 0,09 \\
\hline$\beta_{\eta_{1} 0}$ & grupo controle, mão pref., aval. final e seq. treinada & $-0,01$ & 0,01 \\
\hline$\beta_{\eta_{1} 1}$ & efeito do estágio inicial & $-0,02$ & 0,03 \\
\hline$\beta_{\eta_{1} 2}$ & efeito do estágio avançado & 0,03 & 0,02 \\
\hline$\beta_{\eta_{1} D}$ & efeito da condição dificultadora & 0,01 & 0,01 \\
\hline$\beta_{\eta_{1} N}$ & efeito da mão não preferencial & $-0,01$ & 0,02 \\
\hline$\beta_{\eta_{1} C}$ & efeito da seqüência controle & 0,01 & 0,02 \\
\hline$\beta_{\eta_{1}(1 * D)}$ & efeito do estágio inicial e condição dificultadora & 0,02 & 0,03 \\
\hline$\beta_{\eta_{1}(1 * N)}$ & efeito do estágio inicial e mão não preferencial & 0,08 & 0,03 \\
\hline$\beta_{\eta_{1}(1 * C)}$ & efeito do estágio inicial e seqüência controle & $-0,03$ & 0,02 \\
\hline$\beta_{\eta_{1}(2 * D)}$ & efeito do estágio avançado e condição dificultadora & 0,08 & 0,03 \\
\hline$\beta_{\eta_{1}(2 * N)}$ & efeito do estágio avançado e mão não preferencial & 0,12 & 0,04 \\
\hline$\beta_{\eta_{1}(2 * C)}$ & efeito do estágio avançado e seqüência controle & $-0,04$ & 0,03 \\
\hline$\beta_{\eta_{1}(D * N)}$ & efeito da condição dificultadora e mão não preferencial & 0,01 & 0,03 \\
\hline$\beta_{\eta_{1}(D * C)}$ & efeito da condição dificultadora e sequência controle & $-0,01$ & 0,01 \\
\hline$\beta_{\eta_{1}(N * C)}$ & efeito da mão não preferencial e sequência controle & $-0,02$ & 0,03 \\
\hline
\end{tabular}

Códigos: pref.: preferencial, aval.: avaliação e seq.: seqüência 
Tabela 3.10: Estimativas dos parâmetros $\boldsymbol{\beta}_{\alpha}$ e $\boldsymbol{\beta}_{\delta}$ sob o modelo Beta-Binomial/Poisson-Gama com enlace gerado pelas probabilidades individuais de sucesso inicial e erros padrões

\begin{tabular}{llcc}
\hline Parâmetro & Relacionado ao & Estimativa & Erro Padrão \\
\hline$\beta_{\alpha 0}$ & grupo controle, mão preferencial & 1,03 & 0,09 \\
$\beta_{\alpha 1}$ & efeito do estágio inicial & 0,10 & 0,13 \\
$\beta_{\alpha 2}$ & efeito do estágio avançado & $-0,09$ & 0,20 \\
$\beta_{\alpha N}$ & efeito da mão não preferencial & $-0,04$ & 0,10 \\
$\beta_{\alpha(1 * N)}$ & efeito do estágio inicial e mão não preferencial & $-0,11$ & 0,21 \\
$\beta_{\alpha(2 * N)}$ & efeito do estágio avançado e mão não preferencial & 0,29 & 0,24 \\
\hline$\beta_{\delta 0}$ & grupo controle, mão preferencial & $-1,53$ & 0,48 \\
$\beta_{\delta 1}$ & efeito do estágio inicial & $-0,48$ & 0,75 \\
$\beta_{\delta 2}$ & efeito do estágio avançado & 1,02 & 0,70 \\
$\beta_{\delta N}$ & efeito da mão não preferencial & $-1,13$ & 0,63 \\
$\beta_{\delta(1 * N)}$ & efeito do estágio inicial e mão não preferencial & 2,40 & 1,01 \\
$\beta_{\delta(2 * N)}$ & efeito do estágio avançado e mão não preferencial & 0,05 & 1,08 \\
\hline
\end{tabular}




\section{Capítulo 4}

\section{Discussão}

O modelo Beta-Binomial/Poisson-Gama generaliza o modelo Beta-Binomial/Poisson p-variado, permitindo a introdução de covariâncias diferentes entre duas contagens de totais (quaisquer que sejam tais contagens) e permite a existência de sobredipersão para as contagens de totais. O modelo Beta-Binomial/Poisson-Gama com enlace gerado pelas probabilidades esperadas de sucesso permite, além disso, relacionar o total de tentativas com a probabilidade de sucesso. O modelo Beta-Binomial/Poisson-Gama com enlace gerado pelas probabilidades individuais de sucesso generaliza ainda a relação entre a probabilidade de sucesso com o total de tentativas, permitindo que tal relação seja diferente nos $p$ instantes de observação. Porém, computacionalmente, este modelo ainda não é viável. Para viabilizá-lo, em futuros trabalhos, podemos usar outras técnicas de estimação para seus parâmetros, como técnicas bayesianas.

Os modelos Beta-Binomial/Poisson p-variado, Beta-Binomial/Poisson-Gama e Beta-Binomial/Poisson-Gama com enlaces também podem ser usados para outras situações. Por exemplo, considerando o conjunto de dados usado neste trabalho, podemos comparar as avaliações inicial, final e sob a condição dificultadora simultaneamente. Tal comparação pode ser feita de maneira direta nos modelos baseados na distribuição Poisson-Gama, bastando para isso incluir parâmetros nos vetores; enquanto que para realizar tal comparação usando o modelo baseado na distribuição Poisson p-variada, seria necessário reescrevê-lo, já que precisaríamos um novo vetor de parâmetros para acomodar um novo instante de avaliação. 
Podemos ainda usar tais modelos para outras aplicações de contagens bivariadas repetidas. Um exemplo disso seria o estudo apresentado em Singer et. al. (1987) sobre a reprodução de caramujos ligados ao processo de transmissão da esquistossomose. Um dos objetivos do estudo era verificar se havia diferença entre o número de ovos postos e, entre estes, quando de fato eclodiam, quando um mesmo grupo de caramujos punha ovos isoladamente (autofecundação) e porteriormente, acasalando-se.

Por fim, outras generalizações dos modelos apresentados ainda são possíveis. Por exemplo, para um caso em que considerássemos que as tentativas possam ter sido realizadas de maneira correta, satisfatória ou incorreta. Nesse caso, poderíamos generalizar os modelos apresentados para um baseado na distribuição Dirichlet-Multinomial/PoissonGama. 


\section{Apêndice A}

\section{Correlações amostrais}

Tabela A.1: Coeficientes de correlação entre as contagens na avaliação final e naquela realizada sob a condição dificultadora para participantes do grupo controle usando a mão não preferencial

\begin{tabular}{|c|c|c|c|c|c|c|c|c|c|c|}
\hline & & & \multicolumn{4}{|c|}{ Avaliação final } & \multicolumn{4}{|c|}{ Condição Dificultadora } \\
\hline & & & \multicolumn{2}{|c|}{ Seq. treinada } & \multicolumn{2}{|c|}{ Seq. controle } & \multicolumn{2}{|c|}{ Seq. treinada } & \multicolumn{2}{|c|}{ Seq. controle } \\
\hline & & & Suc. & Tent. & Suc. & Tent. & Suc. & Tent. & Suc. & Tent. \\
\hline \multirow{4}{*}{$\begin{array}{l}\text { Avaliação } \\
\text { final }\end{array}$} & Seq. & Suc. & 1 & & & & & & & \\
\hline & trein. & Tent. & 0,97 & 1 & & & & & & \\
\hline & Seq. & Suc. & 0,71 & 0,66 & 1 & & & & & \\
\hline & controle & Tent. & 0,82 & 0,76 & 0,89 & 1 & & & & \\
\hline Condição & Seq. & Suc. & 0,84 & 0,85 & 0,54 & 0,75 & 1 & & & \\
\hline \multirow[t]{3}{*}{ Dificultadora } & trein. & Tent. & 0,72 & 0,73 & 0,62 & 0,78 & 0,89 & 1 & & \\
\hline & Seq. & Suc. & 0,35 & 0,34 & $-0,05$ & 0,02 & 0,40 & $-0,01$ & 1 & \\
\hline & controle & Tent. & 0,10 & 0,01 & 0,11 & 0,13 & 0,29 & 0,19 & 0,54 & 1 \\
\hline
\end{tabular}

Códigos: Suc.: Sucessos, Tent.: Tentativas e Seq.: seqüência 
Tabela A.2: Coeficientes de correlação entre as contagens na avaliação final e naquela realizada sob a condição dificultadora para pacientes em estágio inicial da doença usando a mão preferencial

\begin{tabular}{|c|c|c|c|c|c|c|c|c|c|c|}
\hline & & & \multicolumn{4}{|c|}{ Avaliação final } & \multicolumn{4}{|c|}{ Condição Dificultadora } \\
\hline & & & \multicolumn{2}{|c|}{ Seq. treinada } & \multicolumn{2}{|c|}{ Seq. controle } & \multicolumn{2}{|c|}{ Seq. treinada } & \multicolumn{2}{|c|}{ Seq. controle } \\
\hline & & & Suc. & Tent. & Suc. & Tent. & Suc. & Tent. & Suc. & Tent. \\
\hline \multirow{4}{*}{$\begin{array}{c}\text { Avaliação } \\
\text { final }\end{array}$} & Seq. & Suc. & 1 & & & & & & & \\
\hline & trein. & Tent. & 0,84 & 1 & & & & & & \\
\hline & Seq. & Suc. & 0,09 & $-0,14$ & 1 & & & & & \\
\hline & controle & Tent. & 0,27 & 0,23 & 0,88 & 1 & & & & \\
\hline Condição & Seq. & Suc. & 0,43 & 0,39 & 0,78 & 0,93 & 1 & & & \\
\hline \multirow[t]{3}{*}{ Dificultadora } & trein. & Tent. & 0,35 & 0,38 & 0,50 & 0,58 & 0,42 & 1 & & \\
\hline & Seq. & Suc. & $-0,31$ & $-0,40$ & 0,79 & 0,74 & 0,58 & 0,09 & 1 & \\
\hline & controle & Tent. & 0,38 & 0,48 & 0,60 & 0,75 & 0,75 & 0,85 & 0,20 & 1 \\
\hline
\end{tabular}

Códigos: Suc.: Sucessos, Tent.: Tentativas e Seq.: seqüência

Tabela A.3: Coeficientes de correlação entre as contagens na avaliação final e naquela realizada sob a condição dificultadora para pacientes em estágio inicial da doença usando a mão não preferencial

\begin{tabular}{|c|c|c|c|c|c|c|c|c|c|c|}
\hline & & & \multicolumn{4}{|c|}{ Avaliação final } & \multicolumn{4}{|c|}{ Condição Dificultadora } \\
\hline & & & \multicolumn{2}{|c|}{ Seq. treinada } & \multicolumn{2}{|c|}{ Seq. controle } & \multicolumn{2}{|c|}{ Seq. treinada } & \multicolumn{2}{|c|}{ Seq. controle } \\
\hline & & & Suc. & Tent. & Suc. & Tent. & Suc. & Tent. & Suc. & Tent. \\
\hline Avaliação & Seq. & Suc. & 1 & & & & & & & \\
\hline \multirow[t]{3}{*}{ final } & trein. & Tent. & 0,99 & 1 & & & & & & \\
\hline & Seq. & Suc. & 0,83 & 0,85 & 1 & & & & & \\
\hline & controle & Tent. & 0,79 & 0,84 & 0,94 & 1 & & & & \\
\hline Condição & Seq. & Suc. & 0,76 & 0,81 & 0,79 & 0,93 & 1 & & & \\
\hline \multirow[t]{3}{*}{ Dificultadora } & trein. & Tent. & 0,75 & 0,80 & 0,84 & 0,97 & 0,99 & 1 & & \\
\hline & Seq. & Suc. & 0,85 & 0,88 & 0,72 & 0,84 & 0,93 & 0,89 & 1 & \\
\hline & controle & Tent. & 0,88 & 0,91 & 0,97 & 0,97 & 0,91 & 0,93 & 0,85 & 1 \\
\hline
\end{tabular}

Códigos: Suc.: Sucessos, Tent.: Tentativas e Seq.: sequiência 
Tabela A.4: Coeficientes de correlação entre as contagens na avaliação final e naquela realizada sob a condição dificultadora para pacientes em estágio avançado da doença usando a mão preferencial

\begin{tabular}{|c|c|c|c|c|c|c|c|c|c|c|}
\hline & & & \multicolumn{4}{|c|}{ Avaliação final } & \multicolumn{4}{|c|}{ Condição Dificultadora } \\
\hline & & & \multicolumn{2}{|c|}{ Seq. treinada } & \multicolumn{2}{|c|}{ Seq. controle } & \multicolumn{2}{|c|}{ Seq. treinada } & \multicolumn{2}{|c|}{ Seq. controle } \\
\hline & & & Suc. & Tent. & Suc. & Tent. & Suc. & Tent. & Suc. & Tent. \\
\hline \multirow{4}{*}{$\begin{array}{l}\text { Avaliação } \\
\text { final }\end{array}$} & Seq. & Suc. & 1 & & & & & & & \\
\hline & trein. & Tent. & 0,97 & 1 & & & & & & \\
\hline & Seq. & Suc. & 0,88 & 0,88 & 1 & & & & & \\
\hline & controle & Tent. & 0,98 & 0,97 & 0,95 & 1 & & & & \\
\hline Condição & Seq. & Suc. & 0,83 & 0,82 & 0,86 & 0,88 & 1 & & & \\
\hline \multirow[t]{3}{*}{ Dificultadora } & trein. & Tent. & 0,82 & 0,83 & 0,88 & 0,87 & 0,98 & 1 & & \\
\hline & Seq. & Suc. & 0,53 & 0,59 & 0,73 & 0,59 & 0,44 & 0,52 & 1 & \\
\hline & controle & Tent. & 0,46 & 0,54 & 0,61 & 0,50 & 0,44 & 0,53 & 0,94 & 1 \\
\hline
\end{tabular}

Códigos: Suc.: Sucessos, Tent.: Tentativas e Seq.: seqüência

Tabela A.5: Coeficientes de correlação entre as contagens na avaliação final e naquela realizada sob a condição dificultadora para pacientes em estágio avançado da doença usando a mão não preferencial

\begin{tabular}{|c|c|c|c|c|c|c|c|c|c|c|}
\hline & & & \multicolumn{4}{|c|}{ Avaliação final } & \multicolumn{4}{|c|}{ Condição Dificultadora } \\
\hline & & & \multicolumn{2}{|c|}{ Seq. treinada } & \multicolumn{2}{|c|}{ Seq. controle } & \multicolumn{2}{|c|}{ Seq. treinada } & \multicolumn{2}{|c|}{ Seq. controle } \\
\hline & & & Suc. & Tent. & Suc. & Tent. & Suc. & Tent. & Suc. & Tent. \\
\hline \multirow{4}{*}{$\begin{array}{l}\text { Avaliação } \\
\text { final }\end{array}$} & Seq. & Suc. & 1 & & & & & & & \\
\hline & trein. & Tent. & 0,98 & 1 & & & & & & \\
\hline & Seq. & Suc. & 0,22 & 0,16 & 1 & & & & & \\
\hline & controle & Tent. & 0,05 & 0,22 & 0,37 & 1 & & & & \\
\hline Condição & Seq. & Suc. & 0,96 & 0,95 & $-0,05$ & $-0,08$ & 1 & & & \\
\hline \multirow[t]{3}{*}{ Dificultadora } & trein. & Tent. & 0,98 & 0,98 & 0,04 & 0,02 & 0,99 & 1 & & \\
\hline & Seq. & Suc. & 0,24 & 0,42 & 0,20 & 0,96 & 0,16 & 0,24 & 1 & \\
\hline & controle & Tent. & 0,79 & 0,86 & $-0,37$ & 0,05 & 0,91 & 0,89 & 0,33 & 1 \\
\hline
\end{tabular}

Códigos: Suc.: Sucessos, Tent.: Tentativas e Seq.: sequiência 


\section{Apêndice B}

\section{Derivadas sob o modelo Poisson-Gama}

$$
\begin{gathered}
\frac{\partial L}{\partial \boldsymbol{\beta}_{\lambda}}=\mathbf{Z}_{\lambda}^{\prime} \mathbf{L}\left[\mathbf{L}^{-1} \mathbf{n}-\left(\mathbf{I}_{p} \otimes \mathbf{B}^{-1}\right)\left(\mathbf{1}_{p} \otimes \mathbf{a}\right)\right] \\
\frac{\partial L}{\partial \boldsymbol{\beta}_{\alpha}}=\mathbf{Z}_{\alpha}^{\prime} \mathbf{M}\left[\mathbf{c}-\mathbf{D}^{-1} \log (\mathbf{b})\right] \text { e } \frac{\partial L}{\partial \boldsymbol{\beta}_{\delta}}=\mathbf{Z}_{\delta}^{\prime}\left[\mathbf{D e}+\mathbf{D}^{-1} \mathbf{M l o g}(\mathbf{b})-\mathbf{B}^{-1} \mathbf{L}_{s} \mathbf{a}\right] \\
\frac{\partial^{2} L}{\partial \boldsymbol{\beta}_{\lambda} \partial \boldsymbol{\beta}_{\lambda}^{\prime}}=\mathbf{Z}_{\lambda}^{\prime} \mathbf{L}\left[\mathbf{I}_{p} \otimes\left(\mathbf{A B}^{-1}\right)\right]\left\{\mathbf{L}\left[\mathbf{I}_{p} \otimes\left(\mathbf{D B}{ }^{-1}\right)\right]-\mathbf{I}_{m p}\right\} \mathbf{Z}_{\lambda}, \\
\frac{\partial^{2} L}{\partial \boldsymbol{\beta}_{\lambda} \partial \boldsymbol{\beta}_{\alpha}^{\prime}}=-\mathbf{Z}_{\lambda}^{\prime} \mathbf{L}\left[\mathbf{I}_{p} \otimes\left(\mathbf{M B}^{-1}\right)\right]\left(\mathbf{1}_{p} \otimes \mathbf{Z}_{\alpha}\right), \\
\frac{\partial^{2} L}{\partial \boldsymbol{\beta}_{\lambda} \partial \boldsymbol{\beta}_{\delta}^{\prime}}=-\mathbf{Z}_{\lambda}^{\prime} \mathbf{L}\left[\mathbf{I}_{p} \otimes\left(\mathbf{D B} \mathbf{B}^{-2}\right)\right]\left[\mathbf{I}_{p} \otimes\left(\mathbf{N}_{s}-\mathbf{M} \mathbf{L}_{s}\right)\right]\left(\mathbf{1}_{p} \otimes \mathbf{Z}_{\delta}\right) \\
\frac{\partial^{2} L}{\partial \boldsymbol{\beta}_{\alpha} \partial \boldsymbol{\beta}_{\alpha}^{\prime}}=\mathbf{Z}_{\alpha}^{\prime} \mathbf{M}\left[\mathbf{C}-\mathbf{D}^{-1} \log (\mathbf{B})-\mathbf{M F}\right] \mathbf{Z}_{\alpha}, \\
\frac{\partial^{2} L}{\partial \boldsymbol{\beta}_{\alpha} \partial \boldsymbol{\beta}_{\delta}^{\prime}}=\mathbf{Z}_{\alpha}^{\prime} \mathbf{M D}\left[-\mathbf{J}-\mathbf{D}^{-1} \mathbf{B}^{-1} \mathbf{L}_{s}+\mathbf{D}^{-2} \log (\mathbf{B})\right] \mathbf{Z}_{\delta} \mathrm{e} \\
\frac{\partial^{2} L}{\partial \boldsymbol{\beta}_{\delta} \partial \boldsymbol{\beta}_{\delta}^{\prime}}=\mathbf{Z}_{\delta}^{\prime} \mathbf{D}\left\{\mathbf{E}-\mathbf{D Q}+\mathbf{M D}^{-1} \mathbf{B}^{-1} \mathbf{L}_{s}-\mathbf{D}^{-2} \mathbf{M l o g}(\mathbf{B})-\mathbf{B}^{-2} \mathbf{L}_{s}\left[\mathbf{N}_{s}-\mathbf{M L}_{s}\right]\right\} \mathbf{Z}_{\delta}
\end{gathered}
$$

com

$$
\mathbf{a}=\left(a_{1}, \ldots, a_{g}, \ldots, a_{m}\right)^{\prime}, \quad a_{g}=\delta\left(\mathbf{z}_{\delta g}\right)\left[\sum_{h=1}^{p} n_{g h}\right]+\alpha\left(\mathbf{z}_{\alpha g}\right),
$$




$$
\begin{aligned}
& \mathbf{A}=\operatorname{diag}\left(a_{g}\right), \\
& \mathbf{B}=\operatorname{diag}\left(b_{g}\right), \quad b_{g}=\delta\left(\mathbf{z}_{\delta g}\right)\left[\sum_{h=1}^{p} \lambda\left(\mathbf{z}_{\lambda g h}\right)\right]+1, \\
& \log (\mathbf{b})=\left(\log \left(b_{1}\right), \ldots, \log \left(b_{g}\right), \ldots, \log \left(b_{m}\right)\right)^{\prime}, \\
& \log (\mathbf{B})=\operatorname{diag}\left[\log \left(b_{g}\right)\right] \\
& \mathbf{c}=\left(c_{1}, \ldots, c_{g}, \ldots, c_{m}\right)^{\prime}, \quad c_{g}=\sum_{u=0}^{\Sigma_{h=1}^{p} n_{g h}-1} \frac{1}{\alpha\left(\mathbf{z}_{\alpha g}\right)+u \delta\left(\mathbf{z}_{\delta g}\right)}, \\
& \mathbf{C}=\operatorname{diag}\left(c_{g}\right) \text {, } \\
& \mathbf{e}=\left(e_{1}, \ldots, e_{g}, \ldots, e_{m}\right)^{\prime}, \quad e_{g}=\sum_{u=0}^{\Sigma_{h=1}^{p} n_{g h}-1} \frac{u}{\alpha\left(\mathbf{z}_{\alpha g}\right)+u \delta\left(\mathbf{z}_{\delta g}\right)}, \\
& \mathbf{E}=\operatorname{diag}\left(e_{g}\right), \\
& \mathbf{F}=\operatorname{diag}\left(f_{g}\right), f_{g}=\sum_{u=0}^{\Sigma_{h=1}^{p} n_{g h}-1} \frac{1}{\left[\alpha\left(\mathbf{z}_{\alpha g}\right)+u \delta\left(\mathbf{z}_{\delta g}\right)\right]^{2}}, \\
& \mathbf{J}=\operatorname{diag}\left(j_{g}\right), \quad j_{g}=\sum_{u=0}^{\Sigma_{h=1}^{p} n_{g h}-1} \frac{u}{\left[\alpha\left(\mathbf{z}_{\alpha g}\right)+u \delta\left(\mathbf{z}_{\delta g}\right)\right]^{2}} \\
& \mathbf{Q}=\operatorname{diag}\left(q_{g}\right), q_{g}=\sum_{u=0}^{\Sigma_{h=1}^{p} n_{g h}-1}\left[\frac{u}{\alpha\left(\mathbf{z}_{\alpha g}\right)+u \delta\left(\mathbf{z}_{\delta g}\right)}\right]^{2} \\
& \mathbf{n}=\left(n_{11}, \ldots, n_{g h}, \ldots, n_{m p}\right)^{\prime}, \\
& \mathbf{N}_{s}=\operatorname{diag}\left[\sum_{h=1}^{p} n_{g h}\right] \\
& \mathbf{L}=\operatorname{diag}\left[\lambda\left(\mathbf{z}_{\lambda g h}\right)\right] \text {, } \\
& \mathbf{L}_{s}=\operatorname{diag}\left[\sum_{h=1}^{p} \lambda\left(\mathbf{z}_{\lambda g h}\right)\right] \text {, } \\
& \mathbf{M}=\operatorname{diag}\left[\alpha\left(\mathbf{z}_{\alpha g}\right)\right] \text {, } \\
& \mathbf{D}=\operatorname{diag}\left[\delta\left(\mathbf{z}_{\delta g}\right)\right], \\
& \mathbf{Z}_{\lambda}=\left(\mathbf{z}_{\lambda 11}^{\prime}, \ldots, \mathbf{z}_{\lambda g h}^{\prime}, \ldots, \mathbf{z}_{\lambda m p}^{\prime}\right)^{\prime}, \\
& \mathbf{Z}_{\alpha}=\left(\mathbf{z}_{\alpha 1}^{\prime}, \ldots, \mathbf{z}_{\alpha g}^{\prime}, \ldots, \mathbf{z}_{\alpha m}^{\prime}\right)^{\prime} \\
& \mathbf{Z}_{\delta}=\left(\mathbf{z}_{\delta 1}^{\prime}, \ldots, \mathbf{z}_{\delta g}^{\prime}, \ldots, \mathbf{z}_{\delta m}^{\prime}\right)^{\prime} .
\end{aligned}
$$




\section{Apêndice $C$}

\section{Derivadas sob o modelo}

Beta-Binomial/Poisson-Gama com

enlace gerado pelas probabilidades

esperadas de sucesso

$$
\begin{aligned}
& \frac{\partial L}{\partial \boldsymbol{\beta}_{\mu}}=\mathbf{Z}_{\mu}^{\prime} \mathbf{M}\left(\mathbf{I}_{m p}-\mathbf{M}\right)\left\{\mathbf{T}^{-1}\left(-\mathbf{d g}_{1}+\mathbf{d g}_{2}+\mathbf{d g}_{3}-\mathbf{d g}_{4}\right)+\right. \\
& \left.\mathbf{I}_{p} \otimes\left[\mathbf{A E}_{1}\left(\mathbf{D} \overline{\mathbf{M}}\left(\mathbf{I}_{m}-\overline{\mathbf{M}}\right) p\right)^{-1}\left(\mathbf{d g}_{7}-\mathbf{d g}_{8}-\log \left(\mathbf{D l}_{s}+\mathbf{1}_{m}\right)\right)\right]\right\} \\
& \frac{\partial L}{\partial \boldsymbol{\beta}_{\theta}}=\mathbf{Z}_{\theta}^{\prime} \mathbf{T}^{-1}\left[-\mathbf{d g}_{5}+(\mathbf{M}) \mathbf{d g}_{1}+\left(\mathbf{I}_{m p}-\mathbf{M}\right) \mathbf{d g}_{2}-(\mathbf{M}) \mathbf{d g}_{3}-\left(\mathbf{I}_{m p}-\mathbf{M}\right) \mathbf{d g}_{4}+\mathbf{d g}_{6}\right] \\
& \frac{\partial L}{\partial \boldsymbol{\beta}_{\lambda}}=\mathbf{Z}_{\lambda}^{\prime}\left\{\mathbf{n}-\mathbf{I}_{p} \otimes\left[\left(\mathbf{D N}_{s}+\mathbf{A}\right)\left(\mathbf{D L}_{s}+\mathbf{I}_{m}\right)^{-1}\right] \mathbf{l}\right\} \\
& \frac{\partial L}{\partial \boldsymbol{\beta}_{\eta_{1}}}=\mathbf{Z}_{\eta_{1}}^{\prime} \mathbf{A D}^{-1} \log \left(\frac{\overline{\mathbf{M}}}{\mathbf{I}_{m}-\overline{\mathbf{M}}}\right)\left\{\mathbf{d g}_{7}-\mathbf{d g}_{8}-\log \left(\mathbf{D l}_{s}+\mathbf{1}_{m}\right)\right\} \\
& \frac{\partial L}{\partial \boldsymbol{\beta}_{\delta}}=\mathbf{Z}_{\delta}^{\prime} \mathbf{D}^{-1}\left\{\mathbf{A}\left[-\mathbf{d g}_{7}+\mathbf{d g}_{8}+\log \left(\mathbf{D l} \mathbf{l}_{s}+\mathbf{1}_{m}\right)\right]+\left(\mathbf{N}_{s}-\mathbf{L}_{s} \mathbf{A}\right)\left(\mathbf{D L}_{s}+\mathbf{I}_{m}\right)^{-1} \mathbf{d}\right\} \\
& \frac{\partial^{2} L}{\partial \boldsymbol{\beta}_{\mu} \partial \boldsymbol{\beta}_{\mu}^{\prime}}=\mathbf{Z}_{\mu}^{\prime} \mathbf{M}\left(\mathbf{I}_{m p}-\mathbf{M}\right)\left\{( \mathbf { I } _ { m p } - 2 \mathbf { M } ) \left[\mathbf{T}^{-1}\left(-\mathbf{D G}_{1}+\mathbf{D G}_{2}+\mathbf{D G}_{3}-\mathbf{D G}_{4}\right)+\right.\right. \\
& \left.\mathbf{I}_{p} \otimes\left[\mathbf{A E}_{1}\left(\mathbf{D} \overline{\mathbf{M}}\left(\mathbf{I}_{m}-\overline{\mathbf{M}}\right) p\right)^{-1}\left(\mathbf{D G}_{7}-\mathbf{D G}_{8}-\log \left(\mathbf{D L}_{s}+\mathbf{1}_{m}\right)\right)\right]\right]+ \\
& \mathbf{M}\left(\mathbf{I}_{m p}-\mathbf{M}\right)\left[\mathbf{T}^{-2}\left(-\mathbf{T G}_{1}-\mathbf{T G}_{2}+\mathbf{T G}_{3}+\mathbf{T G}_{4}\right)+\right.
\end{aligned}
$$




$$
\begin{aligned}
& \mathbf{I}_{p} \otimes\left[\mathbf { A E } _ { 1 } \mathbf { D } ^ { - 1 } ( \overline { \mathbf { M } } ( \mathbf { I } _ { m } - \overline { \mathbf { M } } ) p ) ^ { - 2 } \left(\left(\mathbf{D G}_{7}-\mathbf{D G}_{8}-\log \left(\mathbf{D L}_{s}+\mathbf{I}_{m}\right)\right)\left(\mathbf{E}_{1}-\mathbf{I}_{m}+2 \overline{\mathbf{M}}\right)+\right.\right. \\
& \left.\left.\left.\left.\mathbf{A E}_{1} \mathbf{D}^{-2}\left(\mathbf{T G}_{7}-\mathbf{T G}_{8}\right)\right)\right]\right]\right\} \mathbf{Z}_{\mu} \\
& \frac{\partial^{2} L}{\partial \boldsymbol{\beta}_{\mu} \partial \boldsymbol{\beta}_{\theta}^{\prime}}=\mathbf{Z}_{\mu}^{\prime} \mathbf{M}\left(\mathbf{I}_{m p}-\mathbf{M}\right) \mathbf{T}^{-1}\left\{\mathbf{D G}_{1}-\mathbf{D G}_{2}-\mathbf{D G}_{3}+\mathbf{D G}_{4}+\right. \\
& \left.\mathbf{T}^{-1}\left[(\mathbf{M}) \mathbf{T G}_{1}-\left(\mathbf{I}_{m p}-\mathbf{M}\right) \mathbf{T} \mathbf{G}_{2}-(\mathbf{M}) \mathbf{T} \mathbf{G}_{3}+\left(\mathbf{I}_{m p}-\mathbf{M}\right) \mathbf{T G}_{4}\right]\right\} \mathbf{Z}_{\theta} \\
& \frac{\partial^{2} L}{\partial \boldsymbol{\beta}_{\mu} \partial \boldsymbol{\beta}_{\lambda}^{\prime}}=-\mathbf{Z}_{\mu}^{\prime} \mathbf{M}\left(\mathbf{I}_{m p}-\mathbf{M}\right) \mathbf{L} \mathbf{I}_{p} \otimes\left[\mathbf{A E}_{1}\left(\overline{\mathbf{M}}(\mathbf{I}-\overline{\mathbf{M}})\left(\mathbf{D L} \mathbf{L}_{s}+\mathbf{I}_{m}\right) p\right)^{-1}\right] \mathbf{Z}_{\lambda} \\
& \frac{\partial^{2} L}{\partial \boldsymbol{\beta}_{\mu} \partial \boldsymbol{\beta}_{\eta_{1}}^{\prime}}=\mathbf{Z}_{\mu}^{\prime} \mathbf{M}\left(\mathbf{I}_{m p}-\mathbf{M}\right) \mathbf{I}_{p} \otimes\left\{\mathbf { A } ( \mathbf { D } \overline { \mathbf { M } } ( \mathbf { I } - \overline { \mathbf { M } } ) p ) ^ { - 1 } \left[\left(\mathbf{D G}_{7}-\mathbf{D G}_{8}-\log \left(\mathbf{D} \mathbf{L}_{s}+\mathbf{I}_{m}\right)\right)\right.\right. \\
& \left.\left.\left(\mathbf{I}_{m}+\mathbf{E}_{1} \log \left(\frac{\overline{\mathbf{M}}}{\mathbf{I}-\overline{\mathbf{M}}}\right)\right)+\mathbf{A E}_{1} \mathbf{D}^{-1} \log \left(\frac{\overline{\mathbf{M}}}{\mathbf{I}-\overline{\mathbf{M}}}\right)\left(\mathbf{T G}_{7}-\mathbf{T G}_{8}\right)\right]\right\}\left(\mathbf{1}_{p} \otimes \mathbf{Z}_{\eta_{1}}\right) \\
& \frac{\partial^{2} L}{\partial \boldsymbol{\beta}_{\mu} \partial \boldsymbol{\beta}_{\delta}^{\prime}}=\mathbf{Z}_{\mu}^{\prime} \mathbf{M}\left(\mathbf{I}_{m p}-\mathbf{M}\right) \mathbf{I}_{p} \otimes\left\{\mathbf{A}(\mathbf{D} \overline{\mathbf{M}}(\mathbf{I}-\overline{\mathbf{M}}) p)^{-1}\right. \\
& \left.\left[-\mathbf{D G}_{7}+\mathbf{D G}_{8}+\log \left(\mathbf{D L}_{s}+\mathbf{I}_{m}\right)+\mathbf{A D}^{-1}\left(-\mathbf{T G}_{7}+\mathbf{T G}_{8}\right)-\mathbf{D L}_{s}\left(\mathbf{D L}_{s}+\mathbf{I}_{m}\right)^{-1}\right]\right\}\left(\mathbf{1}_{p} \otimes \mathbf{Z}_{\delta}\right) \\
& \frac{\partial^{2} L}{\partial \boldsymbol{\beta}_{\theta} \partial \boldsymbol{\beta}_{\theta}^{\prime}}=\mathbf{Z}_{\theta}^{\prime} \mathbf{T}^{-1}\left\{\mathbf{D G}_{5}-(\mathbf{M}) \mathbf{D} \mathbf{G}_{1}-\left(\mathbf{I}_{m p}-\mathbf{M}\right) \mathbf{D G}_{2}+(\mathbf{M}) \mathbf{D} \mathbf{G}_{3}+\left(\mathbf{I}_{m p}-\mathbf{M}\right) \mathbf{D G}_{4}-\mathbf{D G}_{6}\right. \\
& \left.+\mathbf{T}^{-1}\left[\mathbf{T G}_{5}-(\mathbf{M})^{2} \mathbf{T} \mathbf{G}_{1}-\left(\mathbf{I}_{m p}-\mathbf{M}\right)^{2} \mathbf{T} \mathbf{G}_{2}+(\mathbf{M})^{2} \mathbf{T} \mathbf{G}_{3}+\left(\mathbf{I}_{m p}-\mathbf{M}\right)^{2} \mathbf{T G}_{4}-\mathbf{T G}_{6}\right]\right\} \mathbf{Z}_{\theta} \\
& \frac{\partial^{2} L}{\partial \boldsymbol{\beta}_{\theta} \partial \boldsymbol{\beta}_{\lambda}^{\prime}}=\frac{\partial^{2} L}{\partial \boldsymbol{\beta}_{\theta} \partial \boldsymbol{\beta}_{\eta_{1}}^{\prime}}=\frac{\partial^{2} L}{\partial \boldsymbol{\beta}_{\theta} \partial \boldsymbol{\beta}_{\delta}^{\prime}}=\mathbf{0} \\
& \frac{\partial^{2} L}{\partial \boldsymbol{\beta}_{\lambda} \partial \boldsymbol{\beta}_{\lambda}^{\prime}}=-\mathbf{Z}_{\lambda}^{\prime} \mathbf{L} \mathbf{I}_{p} \otimes\left[\left(\mathbf{D N}_{s}+\mathbf{A}\right)\left(\mathbf{D L}_{s}+\mathbf{I}_{m}\right)^{-2}\right]\left[\mathbf{I}_{p} \otimes\left(\mathbf{D L}_{s}\right)+\mathbf{I}_{m p}-\mathbf{L}\left(\mathbf{I}_{p} \otimes \mathbf{D}\right)\right] \mathbf{Z}_{\lambda} \\
& \frac{\partial^{2} L}{\partial \boldsymbol{\beta}_{\lambda} \partial \boldsymbol{\beta}_{\eta_{1}}^{\prime}}=-\mathbf{Z}_{\lambda}^{\prime} \mathbf{L} \mathbf{I}_{p} \otimes\left[\mathbf{A}\left(\mathbf{D} \mathbf{L}_{s}+\mathbf{I}_{m}\right)^{-1} \log \left(\frac{\overline{\mathbf{M}}}{\mathbf{I}_{m}-\overline{\mathbf{M}}}\right)\right]\left(\mathbf{1}_{p} \otimes \mathbf{Z}_{\eta_{1}}\right) \\
& \frac{\partial^{2} L}{\partial \boldsymbol{\beta}_{\lambda} \partial \boldsymbol{\beta}_{\delta}^{\prime}}=-\mathbf{Z}_{\lambda}^{\prime} \mathbf{L} \mathbf{I}_{p} \otimes\left[\mathbf{D}\left(\mathbf{D} \mathbf{L}_{s}+\mathbf{I}_{m}\right)^{-2}\left(\mathbf{N}_{s}-\mathbf{A L}_{s}\right)\right]\left(\mathbf{1}_{p} \otimes \mathbf{Z}_{\delta}\right) \\
& \frac{\partial^{2} L}{\partial \boldsymbol{\beta}_{\eta_{1}} \partial \boldsymbol{\beta}_{\eta_{1}}^{\prime}}=\mathbf{Z}_{\eta_{1}}^{\prime} \mathbf{A D}^{-1}\left[\log \left(\frac{\overline{\mathbf{M}}}{\mathbf{I}_{m}-\overline{\mathbf{M}}}\right)\right]^{2}\left\{\mathbf{D G}_{7}-\mathbf{D G}_{8}-\log \left(\mathbf{D L} \mathbf{L}_{s}+\mathbf{I}_{m}\right)+\mathbf{A D}^{-1}\left(\mathbf{T G}_{7}-\mathbf{T G}_{8}\right)\right\} \mathbf{Z}_{\eta_{1}} \\
& \frac{\partial^{2} L}{\partial \boldsymbol{\beta}_{\eta_{1}} \partial \boldsymbol{\beta}_{\delta}^{\prime}}=\mathbf{Z}_{\eta_{1}}^{\prime} \mathbf{A D}^{-1} \log \left(\frac{\overline{\mathbf{M}}}{\mathbf{I}_{m}-\overline{\mathbf{M}}}\right)\left\{-\mathbf{D G}_{7}+\mathbf{D G}_{8}+\log \left(\mathbf{D L} \mathbf{L}_{s}+\mathbf{I}_{m}\right)+\right. \\
& \left.\mathbf{A D}^{-1}\left(-\mathbf{T G}_{7}+\mathbf{T G}_{8}\right)-\mathbf{D L}_{s}\left(\mathbf{D L}_{s}+\mathbf{I}_{m}\right)^{-1}\right\} \mathbf{Z}_{\delta} \\
& \frac{\partial^{2} L}{\partial \boldsymbol{\beta}_{\delta} \partial \boldsymbol{\beta}_{\delta}^{\prime}}=\mathbf{Z}_{\delta}^{\prime} \mathbf{D}^{-1}\left\{-\mathbf{A}\left[-\mathbf{D G}_{7}+\mathbf{D G}_{8}+\log \left(\mathbf{D L}_{s}+\mathbf{I}_{m}\right)\right]+\right.
\end{aligned}
$$




$$
\left.\mathbf{A}^{2} \mathbf{D}^{-1}\left(\mathbf{T G}_{7}-\mathbf{T G}_{8}\right)+\mathbf{D L}_{s}\left(\mathbf{D L}_{s}+\mathbf{I}_{m}\right)^{-2}\left[-\mathbf{D N}_{s}+2 \mathbf{A D L}_{s}+\mathbf{A}\right]\right\} \mathbf{Z}_{\delta}
$$

com

$$
\begin{aligned}
& \operatorname{dg}_{1}=\left(\psi\left(\frac{\mu\left(\mathbf{z}_{\mu 11}\right)}{\theta\left(\mathbf{z}_{\theta 11}\right)}\right), \ldots, \psi\left(\frac{\mu\left(\mathbf{z}_{\mu g h}\right)}{\theta\left(\mathbf{z}_{\theta g h}\right)}\right), \ldots, \psi\left(\frac{\mu\left(\mathbf{z}_{\mu m p}\right)}{\theta\left(\mathbf{z}_{\theta m p}\right)}\right)\right) \\
& \mathbf{D G}_{1}=\operatorname{diag}\left\{\psi\left(\frac{\mu\left(\mathbf{z}_{\mu g h}\right)}{\theta\left(\mathbf{z}_{\theta g h}\right)}\right)\right\} \\
& \operatorname{dg}_{2}=\left(\psi\left(\frac{1-\mu\left(\mathbf{z}_{\mu 11}\right)}{\theta\left(\mathbf{z}_{\theta 11}\right)}\right), \ldots, \psi\left(\frac{1-\mu\left(\mathbf{z}_{\mu g h}\right)}{\theta\left(\mathbf{z}_{\theta g h}\right)}\right), \ldots, \psi\left(\frac{1-\mu\left(\mathbf{z}_{\mu m p}\right)}{\theta\left(\mathbf{z}_{\theta m p}\right)}\right)\right) \\
& \mathbf{D G}_{2}=\operatorname{diag}\left\{\psi\left(\frac{1-\mu\left(\mathbf{z}_{\mu g h}\right)}{\theta\left(\mathbf{z}_{\theta g h}\right)}\right)\right\} \\
& \mathbf{d g}_{3}=\left(\psi\left(\frac{\mu\left(\mathbf{z}_{\mu 11}\right)}{\theta\left(\mathbf{z}_{\theta 11}\right)}+x_{11}\right), \ldots, \psi\left(\frac{\mu\left(\mathbf{z}_{\mu g h}\right)}{\theta\left(\mathbf{z}_{\theta g h}\right)}+x_{g h}\right), \ldots, \psi\left(\frac{\mu\left(\mathbf{z}_{\mu m p}\right)}{\theta\left(\mathbf{z}_{\theta m p}\right)}+x_{m p}\right)\right) \\
& \mathbf{D G}_{3}=\operatorname{diag}\left\{\psi\left(\frac{\mu\left(\mathbf{z}_{\mu g h}\right)}{\theta\left(\mathbf{z}_{\theta g h}\right)}+x_{g h}\right)\right\} \\
& \mathbf{d g}_{4}=\left(\psi\left(\frac{1-\mu\left(\mathbf{z}_{\mu 11}\right)}{\theta\left(\mathbf{z}_{\theta 11}\right)}+n_{11}-x_{11}\right), \ldots, \psi\left(\frac{1-\mu\left(\mathbf{z}_{\mu g h}\right)}{\theta\left(\mathbf{z}_{\theta g h}\right)}+n_{g h}-x_{g h}\right), \ldots,\right. \\
& \left.\psi\left(\frac{1-\mu\left(\mathbf{z}_{\mu m p}\right)}{\theta\left(\mathbf{z}_{\theta m p}\right)}+n_{m p}-x_{m p}\right)\right) \\
& \mathbf{D G}_{4}=\operatorname{diag}\left\{\psi\left(\frac{1-\mu\left(\mathbf{z}_{\mu g h}\right)}{\theta\left(\mathbf{z}_{\theta g h}\right)}+n_{g h}-x_{g h}\right)\right\} \\
& \mathbf{d g}_{5}=\left(\psi\left(\frac{1}{\theta\left(\mathbf{z}_{\theta 11}\right)}\right), \ldots, \psi\left(\frac{1}{\theta\left(\mathbf{z}_{\theta g h}\right)}\right), \ldots, \psi\left(\frac{1}{\theta\left(\mathbf{z}_{\theta m p}\right)}\right)\right) \\
& \mathbf{D G}_{5}=\operatorname{diag}\left\{\psi\left(\frac{1}{\theta\left(\mathbf{z}_{\theta g h}\right)}\right)\right\} \\
& \operatorname{dg}_{6}=\left(\psi\left(\frac{1}{\theta\left(\mathbf{z}_{\theta 11}\right)}+n_{11}\right), \ldots, \psi\left(\frac{1}{\theta\left(\mathbf{z}_{\theta g h}\right)}+n_{g h}\right), \ldots, \psi\left(\frac{1}{\theta\left(\mathbf{z}_{\theta m p}\right)}+n_{m p}\right)\right) \\
& \mathbf{D G}_{6}=\operatorname{diag}\left\{\psi\left(\frac{1}{\theta\left(\mathbf{z}_{\theta g h}\right)}+n_{g h}\right)\right\} \\
& \mathbf{d g}_{7}=\left(\psi\left(\frac{\alpha\left(\mathbf{z}_{\alpha 1}\right)}{\delta\left(\mathbf{z}_{\delta 1}\right)}+\sum_{h=1}^{p} n_{1 h}\right), \ldots, \psi\left(\frac{\alpha\left(\mathbf{z}_{\alpha g h}\right)}{\delta\left(\mathbf{z}_{\delta g h}\right)}+\sum_{h=1}^{p} n_{g h}\right), \ldots, \psi\left(\frac{\alpha\left(\mathbf{z}_{\alpha m p}\right)}{\delta\left(\mathbf{z}_{\delta m}\right)}+\sum_{h=1}^{p} n_{m h}\right)\right) \\
& \mathbf{D G}_{7}=\operatorname{diag}\left\{\psi\left(\frac{\alpha\left(\mathbf{z}_{\alpha g}\right)}{\delta\left(\mathbf{z}_{\delta g}\right)}+\sum_{h=1}^{p} n_{g h}\right)\right\} \\
& \mathbf{d g}_{8}=\left(\psi\left(\frac{\alpha\left(\mathbf{z}_{\alpha 1}\right)}{\delta\left(\mathbf{z}_{\delta 1}\right)}\right), \ldots, \psi\left(\frac{\alpha\left(\mathbf{z}_{\alpha g h}\right)}{\delta\left(\mathbf{z}_{\delta g h}\right)}\right), \ldots, \psi\left(\frac{\alpha\left(\mathbf{z}_{\alpha m p}\right)}{\delta\left(\mathbf{z}_{\delta m}\right)}\right)\right) \\
& \mathbf{D G}_{8}=\operatorname{diag}\left\{\psi\left(\frac{\alpha\left(\mathbf{z}_{\alpha g}\right)}{\delta\left(\mathbf{z}_{\delta g}\right)}\right)\right\}
\end{aligned}
$$




$$
\begin{aligned}
& \mathbf{T G}_{1}=\operatorname{diag}\left\{\psi^{\prime}\left(\frac{\mu\left(\mathbf{z}_{\mu g h}\right)}{\theta\left(\mathbf{z}_{\theta g h}\right)}\right)\right\} \\
& \mathbf{T G}_{2}=\operatorname{diag}\left\{\psi^{\prime}\left(\frac{1-\mu\left(\mathbf{z}_{\mu g h}\right)}{\theta\left(\mathbf{z}_{\theta g h}\right)}\right)\right\} \\
& \mathbf{T G}_{3}=\operatorname{diag}\left\{\psi^{\prime}\left(\frac{\mu\left(\mathbf{z}_{\mu g h}\right)}{\theta\left(\mathbf{z}_{\theta g h}\right)}+x_{g h}\right)\right\} \\
& \mathbf{T G}_{4}=\operatorname{diag}\left\{\psi^{\prime}\left(\frac{1-\mu\left(\mathbf{z}_{\mu g h}\right)}{\theta\left(\mathbf{z}_{\theta g h}\right)}+n_{g h}-x_{g h}\right)\right\} \\
& \mathbf{T G}_{5}=\operatorname{diag}\left\{\psi^{\prime}\left(\frac{1}{\theta\left(\mathbf{z}_{\theta g h}\right)}\right)\right\} \\
& \mathbf{T G}_{6}=\operatorname{diag}\left\{\psi^{\prime}\left(\frac{1}{\theta\left(\mathbf{z}_{\theta g h}\right)}+n_{g h}\right)\right\} \\
& \mathbf{T G}_{7}=\operatorname{diag}\left\{\psi^{\prime}\left(\frac{\alpha\left(\mathbf{z}_{\alpha g}\right)}{\delta\left(\mathbf{z}_{\delta g}\right)}+\sum_{h=1}^{p} n_{g h}\right)\right\} \\
& \mathbf{T G}_{8}=\operatorname{diag}\left\{\psi^{\prime}\left(\frac{\alpha\left(\mathbf{z}_{\alpha g}\right)}{\delta\left(\mathbf{z}_{\delta g}\right)}\right)\right\} \\
& \mathbf{n}=\left(n_{11}, \ldots, n_{g h}, \ldots, n_{m p}\right)^{\prime} \text {, } \\
& \mathbf{N}_{s}=\operatorname{diag}\left\{\sum_{h=1}^{p} n_{g h}\right\} \text {, } \\
& \mathbf{M}=\operatorname{diag}\left\{\mu\left(\mathbf{z}_{\mu g h}\right)\right\} \text {, } \\
& \overline{\mathbf{M}}=\operatorname{diag}\left\{\bar{\mu}_{g}\right\}, \\
& \mathbf{T}=\operatorname{diag}\left\{\theta\left(\mathbf{z}_{\theta g h}\right)\right\}, \\
& \mathbf{l}=\left(\lambda\left(\mathbf{z}_{\lambda 11}\right), \ldots, \lambda\left(\mathbf{z}_{\lambda g h}\right), \ldots, \lambda\left(\mathbf{z}_{\lambda m p}\right)\right), \\
& \mathbf{L}=\operatorname{diag}\left\{\lambda\left(\mathbf{z}_{\lambda g h}\right)\right\}, \\
& \mathbf{l}_{s}=\left(\sum_{h=1}^{p} \lambda\left(\mathbf{z}_{\lambda 11}\right), \ldots, \sum_{h=1}^{p} \lambda\left(\mathbf{z}_{\lambda g h}\right), \ldots, \sum_{h=1}^{p} \lambda\left(\mathbf{z}_{\lambda m p}\right)\right) \text {, } \\
& \mathbf{L}_{s}=\operatorname{diag}\left\{\sum_{h=1}^{p} \lambda\left(\mathbf{z}_{\lambda g h}\right)\right\} \text {, } \\
& \mathbf{E}_{1}=\operatorname{diag}\left\{\eta_{1}\left(\mathbf{z}_{\eta_{1} g}\right)\right\} \text {, } \\
& \mathbf{A}=\operatorname{diag}\left\{\alpha\left(\mathbf{z}_{\alpha g}\right)\right\}, \\
& \mathbf{d}=\left(\delta\left(\mathbf{z}_{\delta 1}\right), \ldots, \delta\left(\mathbf{z}_{\delta g}\right), \ldots, \delta\left(\mathbf{z}_{\delta m}\right)\right), \\
& \mathbf{D}=\operatorname{diag}\left\{\delta\left(\mathbf{z}_{\delta g}\right)\right\},
\end{aligned}
$$




$$
\begin{aligned}
& \mathbf{Z}_{\mu}=\left(\mathbf{z}_{\mu 11}^{\prime}, \ldots, \mathbf{z}_{\mu g h}^{\prime}, \ldots, \mathbf{z}_{\mu m p}^{\prime}\right)^{\prime}, \\
& \mathbf{Z}_{\theta}=\left(\mathbf{z}_{\theta 11}^{\prime}, \ldots, \mathbf{z}_{\theta g h}^{\prime}, \ldots, \mathbf{z}_{\theta m p}^{\prime}\right)^{\prime}, \\
& \mathbf{Z}_{\lambda}=\left(\mathbf{z}_{\lambda 11}^{\prime}, \ldots, \mathbf{z}_{\lambda g h}^{\prime}, \ldots, \mathbf{z}_{\lambda m p}^{\prime}\right)^{\prime}, \\
& \mathbf{Z}_{\eta_{1}}=\left(\mathbf{z}_{\eta_{1} 1}^{\prime}, \ldots, \mathbf{z}_{v g}^{\prime}, \ldots, \mathbf{z}_{\eta_{1} m}^{\prime}\right)^{\prime} \\
& \mathbf{Z}_{\delta}=\left(\mathbf{z}_{\delta 1}^{\prime}, \ldots, \mathbf{z}_{\delta g}^{\prime}, \ldots, \mathbf{z}_{\delta m}^{\prime}\right)^{\prime} .
\end{aligned}
$$




\section{Apêndice D}

\section{Derivadas sob o modelo}

Beta-Binomial/Poisson-Gama com enlace gerado pelas probabilidades individuais de sucesso

$$
\begin{gathered}
\text { Para } \boldsymbol{\beta}_{\mu} \text { e } \boldsymbol{\beta}_{\theta} \\
\frac{\partial L}{\partial \boldsymbol{\beta}_{\mu}}=\mathbf{Z}_{\mu}^{\prime} \mathbf{M}\left(\mathbf{I}_{m p}-\mathbf{M}\right) \mathbf{T}^{-1}\left(-\mathbf{d g}_{1}+\mathbf{d} \mathbf{g}_{2}+\mathbf{p}^{(j)}-\mathbf{q}^{(j)}\right) \\
\frac{\partial L}{\partial \boldsymbol{\beta}_{\theta}}=\mathbf{Z}_{\theta}^{\prime} \mathbf{T}^{-1}\left\{-\mathbf{d g}_{3}+(\mathbf{M}) \mathbf{d g}_{1}+\left(\mathbf{I}_{m p}-\mathbf{M}\right) \mathbf{d g}_{2}-(\mathbf{M}) \mathbf{p}^{(j)}-\left(\mathbf{I}_{m p}-\mathbf{M}\right) \mathbf{q}^{(j)}\right\} \\
\frac{\partial^{2} L}{\partial \boldsymbol{\beta}_{\mu} \partial \boldsymbol{\beta}_{\mu}^{\prime}}=\mathbf{Z}_{\mu}^{\prime} \mathbf{M}\left(\mathbf{I}_{m p}-\mathbf{M}\right) \mathbf{T}^{-1}\left\{\left(\mathbf{I}_{m p}-2 \mathbf{M}\right)\left[-\mathbf{D G} \mathbf{G}_{1}+\mathbf{D G}_{2}+\mathbf{P}^{(j)}-\mathbf{Q}^{(j)}\right]+\right. \\
\left.\mathbf{M}\left(\mathbf{I}_{m p}-\mathbf{M}\right) \mathbf{T}^{-1}\left[-\mathbf{T} \mathbf{G}_{1}-\mathbf{T} \mathbf{G}_{2}\right]\right\} \mathbf{Z}_{\mu} \\
\frac{\partial^{2} L}{\partial \boldsymbol{\beta}_{\mu} \partial \boldsymbol{\beta}_{\theta}^{\prime}}=\mathbf{Z}_{\mu}^{\prime} \mathbf{M}\left(\mathbf{I}_{m p}-\mathbf{M}\right) \mathbf{T}^{-1}\left\{\mathbf{D G} \mathbf{G}_{1}-\mathbf{D G} \mathbf{G}_{2}-\mathbf{P}^{(j)}+\mathbf{Q}^{(j)}+\mathbf{T}^{-1}\left[(\mathbf{M}) \mathbf{T} \mathbf{G}_{1}-\left(\mathbf{I}_{m p}-\mathbf{M}\right) \mathbf{T} \mathbf{G}_{2}\right]\right\} \mathbf{Z}_{\theta} \\
\frac{\partial^{2} L}{\partial \boldsymbol{\beta}_{\theta} \partial \boldsymbol{\beta}_{\theta}^{\prime}}=\mathbf{Z}_{\theta}^{\prime} \mathbf{T}^{-1}\left\{\mathbf{D G} \mathbf{G}_{3}-(\mathbf{M}) \mathbf{D G} \mathbf{G}_{1}-\left(\mathbf{I}_{m p}-\mathbf{M}\right) \mathbf{D} \mathbf{G}_{2}+(\mathbf{M}) \mathbf{P}^{(j)}+\left(\mathbf{I}_{m p}-\mathbf{M}\right) \mathbf{Q}^{(j)}+\right. \\
\left.\mathbf{T}^{-1}\left[\mathbf{T} \mathbf{G}_{3}-(\mathbf{M})^{2} \mathbf{T} \mathbf{G}_{1}-\left(\mathbf{I}_{m p}-\mathbf{M}\right)^{2} \mathbf{T} \mathbf{G}_{2}\right]\right\}
\end{gathered}
$$

Para $\boldsymbol{\beta}_{\eta_{0}}$ e $\boldsymbol{\beta}_{\eta_{1}}$

$$
\frac{\partial L}{\partial \boldsymbol{\beta}_{\eta_{0}}}=\mathbf{Z}_{\eta_{0}}^{\prime}\left\{-\exp \left(\mathbf{E}_{0}\right) \mathbf{r}^{(j)}+\mathbf{n}\right\} \text { e } \frac{\partial L}{\partial \boldsymbol{\beta}_{\eta_{1}}}=\mathbf{Z}_{\eta_{1}}^{\prime}\left\{-\exp \left(\mathbf{E}_{0}\right) \mathbf{u}^{(j)}+\left(\mathbf{P}^{(j)}-\mathbf{Q}^{(j)}\right) \mathbf{n}\right\}
$$




$$
\begin{gathered}
\frac{\partial^{2} L}{\partial \boldsymbol{\beta}_{\eta_{0}} \partial \boldsymbol{\beta}_{\eta_{0}}^{\prime}}=-\mathbf{Z}_{\eta_{0}}^{\prime} \mathbf{R}^{(j)} \exp \left(\mathbf{E}_{0}\right) \mathbf{Z}_{\eta_{0}}, \frac{\partial^{2} L}{\partial \boldsymbol{\beta}_{\eta_{0}} \partial \boldsymbol{\beta}_{\eta_{1}}^{\prime}}=-\mathbf{Z}_{\eta_{0}}^{\prime} \mathbf{U}^{(j)} \exp \left(\mathbf{E}_{0}\right) \mathbf{Z}_{\eta_{1}} \\
\mathrm{e} \frac{\partial^{2} L}{\partial \boldsymbol{\beta}_{\eta_{1}} \partial \boldsymbol{\beta}_{\eta_{1}}^{\prime}}=-\mathbf{Z}_{\eta_{1}}^{\prime} \mathbf{V}^{(j)} \exp \left(\mathbf{E}_{0}\right) \mathbf{Z}_{\eta_{1}}
\end{gathered}
$$

Para $\boldsymbol{\beta}_{\alpha}$ e $\boldsymbol{\beta}_{\delta}$

$$
\begin{gathered}
\frac{\partial L}{\partial \boldsymbol{\beta}_{\alpha}}=\mathbf{Z}_{\alpha}^{\prime} \mathbf{A} \mathbf{D}^{-1}\left\{-\mathbf{d g}_{4}-\log (\mathbf{d})+\mathbf{s}^{(j)}\right\} \mathrm{e} \frac{\partial L}{\partial \boldsymbol{\beta}_{\delta}}=\mathbf{Z}_{\delta}^{\prime} \mathbf{A} \mathbf{D}^{-1}\left\{\mathbf{d g}_{4}+\log (\mathbf{d})-\mathbf{1}_{m}-\mathbf{s}^{(j)}+\mathbf{A}^{-1} \mathbf{w}\right\} \\
\frac{\partial^{2} L}{\partial \boldsymbol{\beta}_{\alpha} \partial \boldsymbol{\beta}_{\alpha}^{\prime}}=\mathbf{Z}_{\alpha}^{\prime} \mathbf{A D}^{-1}\left\{-\mathbf{D} \mathbf{G}_{4}-\log (\mathbf{D})+\mathbf{S}^{(j)}-\left(\mathbf{A} \mathbf{D}^{-1}\right) \mathbf{T} \mathbf{G}_{4}\right\} \mathbf{Z}_{\alpha} \\
\frac{\partial^{2} L}{\partial \boldsymbol{\beta}_{\alpha} \partial \boldsymbol{\beta}_{\delta}^{\prime}}=\mathbf{Z}_{\alpha}^{\prime} \mathbf{A} \mathbf{D}^{-1}\left\{\mathbf{D} \mathbf{G}_{4}+\log (\mathbf{D})-\mathbf{S}^{(j)}+\mathbf{I}_{m}+\left(\mathbf{A} \mathbf{D}^{-1}\right) \mathbf{T} \mathbf{G}_{4}\right\} \mathbf{Z}_{\delta} \\
\frac{\partial^{2} L}{\partial \boldsymbol{\beta}_{\delta} \partial \boldsymbol{\beta}_{\delta}^{\prime}}=\mathbf{Z}_{\delta}^{\prime} \mathbf{A D}^{-1}\left\{-\mathbf{D G} \mathbf{G}_{4}-\log (\mathbf{D})+\mathbf{S}^{(j)}+2 \mathbf{I}_{m}-\mathbf{W} \mathbf{A}^{-1}-\left(\mathbf{A} \mathbf{D}^{-1}\right) \mathbf{T} \mathbf{G}_{4}\right\} \mathbf{Z}_{\delta}
\end{gathered}
$$

com

$$
\begin{aligned}
& \mathbf{p}^{(j)}=\left(p_{11}^{(j)}, \ldots, p_{g h}^{(j)}, \ldots, p_{m p}^{(j)}\right) \\
& \mathbf{P}^{(j)}=\operatorname{diag}\left\{p_{g h}^{(j)}\right\} \\
& \mathbf{q}^{(j)}=\left(q_{11}^{(j)}, \ldots, q_{g h}^{(j)}, \ldots, q_{m p}^{(j)}\right) \\
& \mathbf{Q}^{(j)}=\operatorname{diag}\left\{q_{g h}^{(j)}\right\} \\
& \mathbf{r}^{(j)}=\left(r_{11}^{(j)}\left(\eta_{1}\right), \ldots, r_{g h}^{(j)}\left(\eta_{1}\right), \ldots, r_{m p}^{(j)}\left(\eta_{1}\right)\right) \\
& \mathbf{R}^{(j)}=\operatorname{diag}\left\{r_{g h}^{(j)}\left(\eta_{1}\right)\right\} \\
& \mathbf{s}^{(j)}=\left(s_{1}^{(j)}, \ldots, s_{g}^{(j)}, \ldots, s_{m}^{(j)}\right) \\
& \mathbf{S}^{(j)}=\operatorname{diag}\left\{s_{g}^{(j)}\right\} \\
& \mathbf{w}^{(j)}=\left(w_{1}^{(j)}, \ldots, w_{g}^{(j)}, \ldots, w_{m}^{(j)}\right) \\
& \mathbf{W}^{(j)}=\operatorname{diag}\left\{w_{g}^{(j)}\right\} \\
& \mathbf{u}^{(j)}=\left(u_{11}^{(j)}\left(\eta_{1}\right), \ldots, u_{g h}^{(j)}\left(\eta_{1}\right), \ldots, u_{m p}^{(j)}\left(\eta_{1}\right)\right), \quad u_{g h}^{(j)}\left(\eta_{1}\right)=\frac{d r_{g h}^{(j)}\left(\eta_{1}\right)}{d \eta_{1}} \\
& \mathbf{U}^{(j)}=\operatorname{diag}\left\{u_{g h}^{(j)}\left(\eta_{1}\right)\right\}
\end{aligned}
$$




$$
\begin{aligned}
& \mathbf{V}^{(j)}=\operatorname{diag}\left\{v_{g h}^{(j)}\left(\eta_{1}\right)\right\}, \quad v_{g h}^{(j)}\left(\eta_{1}\right)=\frac{d^{2} r_{g h}^{(j)}\left(\eta_{1}\right)}{d \eta_{1}^{2}} \\
& \operatorname{dg}_{1}=\left(\psi\left(\frac{\mu\left(\mathbf{z}_{\mu 11}\right)}{\theta\left(\mathbf{z}_{\theta 11}\right)}\right), \ldots, \psi\left(\frac{\mu\left(\mathbf{z}_{\mu g h}\right)}{\theta\left(\mathbf{z}_{\theta g h}\right)}\right), \ldots, \psi\left(\frac{\mu\left(\mathbf{z}_{\mu m p}\right)}{\theta\left(\mathbf{z}_{\theta m p}\right)}\right)\right) \\
& \mathbf{D G}_{1}=\operatorname{diag}\left\{\psi\left(\frac{\mu\left(\mathbf{z}_{\mu g h}\right)}{\theta\left(\mathbf{z}_{\theta g h}\right)}\right)\right\} \\
& \mathbf{d g}_{2}=\left(\psi\left(\frac{1-\mu\left(\mathbf{z}_{\mu 11}\right)}{\theta\left(\mathbf{z}_{\theta 11}\right)}\right), \ldots, \psi\left(\frac{1-\mu\left(\mathbf{z}_{\mu g h}\right)}{\theta\left(\mathbf{z}_{\theta g h}\right)}\right), \ldots, \psi\left(\frac{1-\mu\left(\mathbf{z}_{\mu m p}\right)}{\theta\left(\mathbf{z}_{\theta m p}\right)}\right)\right) \\
& \mathbf{D G}_{2}=\operatorname{diag}\left\{\psi\left(\frac{1-\mu\left(\mathbf{z}_{\mu g h}\right)}{\theta\left(\mathbf{z}_{\theta g h}\right)}\right)\right\} \\
& \mathbf{d g}_{3}=\left(\psi\left(\frac{1}{\theta\left(\mathbf{z}_{\theta 11}\right)}\right), \ldots, \psi\left(\frac{1}{\theta\left(\mathbf{z}_{\theta g h}\right)}\right), \ldots, \psi\left(\frac{1}{\theta\left(\mathbf{z}_{\theta m p}\right)}\right)\right) \\
& \mathbf{D G}_{3}=\operatorname{diag}\left\{\psi\left(\frac{1}{\theta\left(\mathbf{z}_{\theta g h}\right)}\right)\right\} \\
& \operatorname{dg}_{4}=\left(\psi\left(\frac{\alpha\left(\mathbf{z}_{\alpha 1}\right)}{\delta\left(\mathbf{z}_{\delta 1}\right)}\right), \ldots, \psi\left(\frac{\alpha\left(\mathbf{z}_{\alpha g h}\right)}{\delta\left(\mathbf{z}_{\delta g h}\right)}\right), \ldots, \psi\left(\frac{\alpha\left(\mathbf{z}_{\alpha m p}\right)}{\delta\left(\mathbf{z}_{\delta m}\right)}\right)\right) \\
& \mathbf{D G}_{4}=\operatorname{diag}\left\{\psi\left(\frac{\alpha\left(\mathbf{z}_{\alpha g}\right)}{\delta\left(\mathbf{z}_{\delta g}\right)}\right)\right\} \\
& \mathbf{T G}_{1}=\operatorname{diag}\left\{\psi^{\prime}\left(\frac{\mu\left(\mathbf{z}_{\mu g h}\right)}{\theta\left(\mathbf{z}_{\theta g h}\right)}\right)\right\} \\
& \mathbf{T G}_{2}=\operatorname{diag}\left\{\psi^{\prime}\left(\frac{1-\mu\left(\mathbf{z}_{\mu g h}\right)}{\theta\left(\mathbf{z}_{\theta g h}\right)}\right)\right\} \\
& \mathbf{T G}_{3}=\operatorname{diag}\left\{\psi^{\prime}\left(\frac{1}{\theta\left(\mathbf{z}_{\theta g h}\right)}\right)\right\} \\
& \mathbf{T G}_{4}=\operatorname{diag}\left\{\psi^{\prime}\left(\frac{\alpha\left(\mathbf{z}_{\alpha g}\right)}{\delta\left(\mathbf{z}_{\delta g}\right)}\right)\right\} \\
& \mathbf{n}=\left(n_{11}, \ldots, n_{g h}, \ldots, n_{m p}\right)^{\prime}, \\
& \mathbf{M}=\operatorname{diag}\left\{\mu\left(\mathbf{z}_{\mu g h}\right)\right\}, \\
& \mathbf{T}=\operatorname{diag}\left\{\theta\left(\mathbf{z}_{\theta g h}\right)\right\}, \\
& \mathbf{E}_{0}=\operatorname{diag}\left\{\eta_{0}\left(\mathbf{z}_{\eta_{0} g h}\right)\right\}, \\
& \mathbf{A}=\operatorname{diag}\left\{\alpha\left(\mathbf{z}_{\alpha g}\right)\right\}, \\
& \mathbf{d}=\left(\delta\left(\mathbf{z}_{\delta 1}\right), \ldots, \delta\left(\mathbf{z}_{\delta g}\right), \ldots, \delta\left(\mathbf{z}_{\delta m}\right)\right), \\
& \mathbf{D}=\operatorname{diag}\left\{\delta\left(\mathbf{z}_{\delta g}\right)\right\},
\end{aligned}
$$




$$
\begin{aligned}
& \mathbf{Z}_{\mu}=\left(\mathbf{z}_{\mu 11}^{\prime}, \ldots, \mathbf{z}_{\mu g h}^{\prime}, \ldots, \mathbf{z}_{\mu m p}^{\prime}\right)^{\prime}, \\
& \mathbf{z}_{\theta}=\left(\mathbf{z}_{\theta 11}^{\prime}, \ldots, \mathbf{z}_{\theta g h}^{\prime}, \ldots, \mathbf{z}_{\theta m p}^{\prime}\right)^{\prime}, \\
& \mathbf{z}_{\eta_{0}}=\left(\mathbf{z}_{\eta_{0} 11}^{\prime}, \ldots, \mathbf{z}_{\eta_{0} g h}^{\prime}, \ldots, \mathbf{z}_{\eta_{0} m p}^{\prime}\right)^{\prime}, \\
& \mathbf{Z}_{\eta_{1}}=\left(\mathbf{z}_{\eta_{1} 11}^{\prime}, \ldots, \mathbf{z}_{\eta_{1} g h}^{\prime}, \ldots, \mathbf{z}_{\eta_{1} m p}^{\prime}\right)^{\prime}, \\
& \mathbf{Z}_{\alpha}=\left(\mathbf{z}_{\alpha 1}^{\prime}, \ldots, \mathbf{z}_{\alpha g}^{\prime}, \ldots, \mathbf{z}_{\alpha m}^{\prime}\right)^{\prime}, \\
& \mathbf{Z}_{\delta}=\left(\mathbf{z}_{\delta 1}^{\prime}, \ldots, \mathbf{z}_{\delta g}^{\prime}, \ldots, \mathbf{z}_{\delta m}^{\prime}\right)^{\prime} .
\end{aligned}
$$




\section{Referências Bibliográficas}

[1] Allen, A.S., Barnhart, H. X. (2002). Joint models for toxicology studies with dosedependent number of implantations. Risk Analysis 2, 1165-1173.

[2] Brooks, S. P., Morgan, J. T., Ridout, M. S., Pack, S. E. (1997). Finite Mixture Models for Proportions. Biometrics 53, 1097-1115.

[3] Comulada, W.S., Weiss, R.E. (2007). On models for Binomial data with random number of trials. Biometrics 63, 610-617.

[4] Dempster, A.P., Laird, N.M., Rubin, D.B. (1977). Maximum Likelihood from Incomplete Data via the EM Algorithm. Journal of the Royal Statistical Society 39, 1-38.

[5] Dunson, D.B. (1998). Dose-dependent number of implants and implications in developmental toxicity. Biometrics 54, 558-569.

[6] Gange, S. J., Munoz, A., Saez, M., Alonso, J. (1996). Use of the Beta-Binomial Distribution to Model the Effect of Policy Changes on Appropriateness of Hospital Stays. Applied Statistics 45, 371-382.

[7] Griffiths, D. A. (1973). Maximum Likelihood Estimation for the Beta-Binomial Distribution and an Application to the Total Number of Cases of a Disease. Biometrics 29, 673-648.

[8] Ho, L.L., Singer, J.M. (1997). Regression models for bivariate counts. Brazilian Journal of Probability and Statistics 11, 175-197. 
[9] Ho, L.L., Singer, J.M. (2001). Generalized least squares methods for bivariate Poisson regression. Communications in Statistics 30, 263-278.

[10] Holgate, P. (1964). Estimation for the Bivariate Poisson Distribution. Biometrika 51, 241-245.

[11] Johnson, N. L., Kotz, S. (1970). Distributions in Statistics: continuous univariate distributions 2. Boston: Houghton Mifflin.

[12] Karlis, D., Ntzoufras, I. (2003). Analysis of sports data by using bivariate Poisson models. The Statistician 52, 381-393.

[13] Kocherlakota, S., Kocherlakota, K. (2001). Regression in the Bivariate Poisson Distribution. Communications in Statistics 30, 815-825.

[14] Lora, M. I. (2004). Modelos de regressão Beta-Binomial/Poisson para contagens bivariadas. Dissertação de mestrado, Instituto de Matemática e Estatística da Universidade de São Paulo, São Paulo.

[15] Lora, M. I., Singer, J. M. (2008). Beta-binomial/Poisson models for repeated bivariate counts. Statistics in Medicine 27, 3366-3381.

[16] Mood, A. M., Graybill, F. A., Boes, D. C. (1974). Introduction to the Theory of Statistics, 3rd edition. Singapore: McGraw-Hill.

[17] Nelder, J. A., McCullagh, P. (1989). Generalized Linear Models, 2nd edition. London: Chapman and Hall.

[18] Nelson, J. F. (1985). Multivariate Gamma-Poisson Models. Journal of the American Statistical Association 392, 828-834.

[19] Piemonte, M. E. P. (2003). Aprendizado motor na doença de Parkinson. Tese de doutorado, Instituto de Psicologia da Universidade de São Paulo, São Paulo.

[20] Rai, K., van Ryzin, J. (1985). A dose-response model for teratological experiments involving quantal responses. Biometrics 41, 1-9. 
[21] Singer, J. M., Paula, L., Machado, F. P.(1987). Relatório de Análise Estatística sobre o Projeto: Alguns aspectos da biologia da reprodução e espermatogênese em animais da espécie Biomphalaia Tenagophila. Setor de Estatística Aplicada, Departamento de Estatística do Instituto de Matemática e Estatística da Universidade de São Paulo, São Paulo.

[22] Singer, J. M., Magalhães, M. N., Petri, C., Aguemi, R. (2002). Relatório de Análise Estatística sobre o Projeto: Característica do Aprendizado Motor na Doença de Parkinson. Centro de Estatística Aplicada, Departamento de Estatística do Instituto de Matemática e Estatística da Universidade de São Paulo, São Paulo.

[23] Wei, G. C. G., Tanner, M. A. (1990). A Monte Carlo Implementation of the EM Algorithm and the Poor Man's Data Augumentation Algorithms . Journal of the American Statistical Association 85, 699-704.

[24] Williams, D. A. (1975). The Analysis of Binary responses from Toxicological Experiments Involving Reproduction and Teratogenicity. Biometrics 31, 949-952.

[25] Zhu, J., Eickhoff, J. C., Kaiser, M. S. (2003). Modeling the Dependence between Number of Trials and Sucess Probability in Beta-Binomial-Poisson Mixture Distributions. Biometrics 59, 955-961. 